\title{
Periodic Orbits Close to Elliptic Tori and Applications to the Three-body Problem
}

\author{
MASSIMILIANO BERTI - LUCA BIASCO - ENRICO VALDINOCI
}

\begin{abstract}
We prove, under suitable non-resonance and non-degeneracy "twist" conditions, a Birkhoff-Lewis type result showing the existence of infinitely many periodic solutions, with larger and larger minimal period, accumulating onto elliptic invariant tori (of Hamiltonian systems). We prove the applicability of this result to the spatial planetary three-body problem in the small eccentricity-inclination regime. Furthermore, we find other periodic orbits under some restrictions on the period and the masses of the "planets". The proofs are based on averaging theory, KAM theory and variational methods.
\end{abstract}

Mathematics Subject Classification (2000): $37 \mathrm{~J} 45$ (primary); 70H08, 70F07, $70 \mathrm{~K} 45$ (secondary).

\section{Contents:}

1. Periodic orbits accumulationg on elliptic tori............ p. 92

2. Study of the resonances and technical Lemmata ............ 96

3. Normal form around an elliptic torus ................. 101

4. Periodic orbits winding along the torus ................ 107

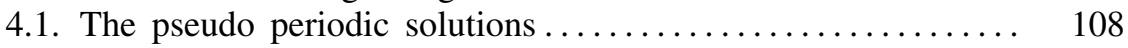

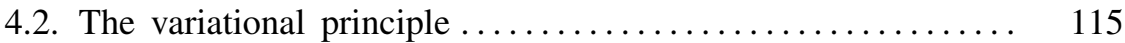

5. The planetary spatial three-body problem ............... 118

5.1. A KAM theorem for elliptic tori ................ 123

5.2. Abundance of periodic solutions in the three-body problem.. 125

6. Periodic orbits near resonant elliptic tori................. 129

7. Appendix: Proof of Proposition $5.1 \ldots \ldots \ldots \ldots \ldots \ldots \ldots \ldots \ldots \ldots \ldots \ldots$

Research supported by M.U.R.S.T. Variational Methods and Nonlinear Differential Equations. Pervenuto alla Redazione il 18 giugno 2003 ed in forma definitiva il 9 febbraio 2004. 


\section{Introduction}

A classical problem in Hamiltonian dynamical systems concerns, since the researches of Poincaré, the existence of periodic orbits in the vicinity of invariant submanifolds.

Poincaré wrote:

“...voici un fait que je n'ai pu démontrer rigoureusement, mais qui me parait pourtant très vraisemblable. Étant données des équations de la forme définie dans le $n .13^{(1)}$ et une solution particulière quelconque de ces équations, on peut toujours trouver une solution périodique (dont la période peut, il est vrai, être très longue), telle que la différence entre les deux solutions soit aussi petite qu'on le veut, pendant un temps aussi long qu'on le veut." ([P], Tome 1, ch. III, a. 36).

This conjecture was often quoted by Birkhoff as a main motivation for his works. In the thirties, Birkhoff and Lewis [B]-[BL]-[L] established the existence of infinitely many periodic solutions in a neighborhood of an elliptic equilibrium whose linear frequencies are sufficiently non-resonant. This theorem also requires a non-degeneracy - "twist"- condition, involving finitely many Taylor coefficients of the Hamiltonian at the equilibrium, and implying the system to be genuinely non-linear. In addition, if the Hamiltonian is sufficiently smooth, KAM theory ensures, in a neighborhood of the equilibrium small enough, the existence of invariant Lagrangian tori filling up a set of positive Lebesgue measure, see [Pö]. Furthermore, close to any KAM torus, it has been proved in [CZ] the existence of infinitely many others periodic orbits with larger and larger minimal period accumulating to the torus itself (as a consequence, the closure of the periodic orbits has positive Lebesgue measure). The result of [CZ] is proved considering the normal form Hamiltonian which describes the dynamics near each torus, checking the "twist condition", and, then, applying the Birkhoff-Lewis type theorem of [Mo].

Actually, Birkhoff and Lewis established also the existence of infinitely many periodic solutions close to a non-constant periodic elliptic solution.

The question of the existence of periodic orbits with larger and larger minimal period clustering to elliptic lower dimensional invariant tori (of dimension greater than one) has not been yet investigated. An invariant torus is called elliptic, or linearly stable, if the linearized system along the torus, possesses purely imaginary eigenvalues.

A main motivation for studying such problem is Celestial Mechanics, which, indeed, inspired the whole development of KAM theory (Arnold [A] devoted one of the fundamental papers of this theory to the planar $n$-body problem).

Consider, in particular, the non-planar planetary three body problem, namely one "star" and two "planets" interacting through a Newtonian gravitational field in the three dimensional space. The masses of the planets are regarded as small

${ }^{(1)}$ Hamilton's equations. 
parameters. According to Poincaré and Delaunay this system is described by a nearly-integrable Hamiltonian on a eight dimensional phase space, equipped with real-analytic action-angle variables. Such system turns out to be properly degenerate, i.e. the integrable limit (in which the three-body problem is described by two decoupled and integrable two-body systems) depends only on two action variables. Hence, in the integrable limit all bounded motions lie on two-dimensional invariant tori supporting periodic or quasi-periodic motions according the ratio between the two frequencies (related, by Kepler's law, to the major semi-axis of the two limiting Keplerian ellipses) is a rational or irrational number. Furthermore, in the small eccentricity-inclination regime (of astronomical interest) these unperturbed two-tori are elliptic: the spatial planetary three-body problem calls for a perturbation theory for continuing elliptic lower-dimensional tori.

In the last years, an exhaustive perturbation theory for elliptic tori has indeed been developed by many authors, see [M], [E], [W], [K], [Pö1], [Pö2], $[\mathrm{Bo}]$ and $[\mathrm{XJ}]$. The persistence of elliptic tori is ensured requiring "Melnikov non-resonance conditions" among the frequencies and further non-degeneracy conditions.

In light of these results, the spatial planetary three body problem (in the small eccentricity-inclination regime) has been recently reexamined in [BCV], where the persistence of two-dimensional elliptic invariant tori -with Diophantine frequencies- has been proved, provided the Keplerian major semi-axes belong to a two dimensional set of positive measure.

Previous works on the spatial planetary three-body problem are [JM], for large inclinations (in this case the two-tori are hyperbolic), and [LR]-[R], for maximal dimensional tori (the proofs are computer assisted).

In the present paper we first prove, under suitable non-resonance and nondegeneracy "twist" conditions, a general Birkhoff-Lewis type result showing the existence of infinitely many periodic solutions, with larger and larger minimal period, accumulating onto elliptic lower dimensional invariant tori, see Theorem 1.1 for the precise statement.

Furthermore we prove the applicability of Theorem 1.1 to the spatial planetary three-body problem, showing the existence of infinitely many periodic $^{(2)}$ solutions minimal accumulating on the elliptic KAM tori of [BCV], see Theorem 0.2. Such periodic orbits revolve around the star close to Keplerian ellipses with small eccentricities and small non-zero mutual inclinations. The verification of the "twist" condition (Lemma 5.1) is based on a KAM analysis.

Finally, in Theorems $0.3,0.4$ we prove the existence of other periodic solutions of the spatial planetary three-body problem, the "periodic analogue" of the elliptic tori of [BCV], see Theorem 6.1 for a more general statement.

We now present a simplified version of our results.

\footnotetext{
(2) The orbits found for the three-body problem are periodic in a suitable reduced set of coordinates.
} We refer to $[\mathrm{BCV}]$ for the physical interpretation of these coordinates. 


\section{Periodic orbits accumulating on elliptic tori}

As already said, the persistence of elliptic invariant tori is ensured assuming "Melnikov non-resonance conditions" among the frequencies. In particular, under the "second order Melnikov conditions" (see precisely (4)), the surviving tori are still elliptic and the normal form Hamiltonian describing the dynamics in a neighborhood is

$$
\mathcal{H}_{*}\left(\mathcal{I}_{*}, \varphi_{*}, Z_{*}, \bar{Z}_{*}\right)=\omega \cdot \mathcal{I}_{*}+\Omega Z_{*} \cdot \bar{Z}_{*}+\sum_{2|k|+|a+\bar{a}| \geq 3} R_{k, a, \bar{a}}^{*}\left(\varphi_{*}\right) \mathcal{I}_{*}^{k} Z_{*}^{a} \bar{Z}_{*}^{\bar{a}},
$$

where $\left(\mathcal{I}_{*}, \varphi_{*}\right) \in \mathbb{R}^{n} \times \mathbb{T}^{n}$ and $\left(Z_{*}, \bar{Z}_{*}\right) \in \mathbb{C}^{2 m}$. In these coordinates $\mathcal{T}:=\left\{\mathcal{I}_{*}=\right.$ $\left.0, \varphi_{*} \in \mathbb{T}^{n}, Z_{*}=\bar{Z}_{*}=0\right\}$ is the invariant and elliptic torus. $\omega \in \mathbb{R}^{n}$ are the torus frequencies and $\Omega \in \mathbb{R}^{m}$ the elliptic one's.

We refer to [JV] and [BHS] for many results concerning the dynamics of an Hamiltonian system close to an elliptic torus, in particular for the existence, under appropriate non-resonance and non-degeneracy conditions, of Cantor families of other invariant tori of dimensions greater than $n$.

Also for proving the existence of periodic orbits accumulating onto the elliptic torus $\mathcal{T}$ we need to assume suitable non-resonance and non-degeneracy "twist" conditions. Roughly (see Theorem 1.1 for the detailed statement) we have:

THEOREM 0.1. Under suitable non-resonance and non-degeneracy conditions, the Hamiltonian system generated by $\mathcal{H}_{*}$ affords infinitely many periodic solutions, with larger and larger minimal period, accumulating on the invariant elliptic torus $\mathcal{T}$.

The precise Theorem 1.1 and a description of its proof are given in Section 1 . Theorem 1.1 implies, in particular, the result of [CZ] for maximal dimensional tori.

\section{The planetary, spatial three-body problem}

Let $\varepsilon>0$ denote the small parameter measuring the ratios between the masses of the planets and the mass of the star (see (93)). The existence of two-dimensional elliptic invariant tori has been proved in [BCV]. Roughly (see Theorem 5.2 for a precise statement and proof) their result states:

THEOREM ([BCV]). The spatial planetary three-body problem affords, for $\varepsilon$ small enough, a family $\mathcal{F}$ of two-dimensional, elliptic invariant tori, traveled with Diophantine quasi-periodic frequencies, provided the osculating Keplerian major semi-axes belong to a two-dimensional set of density close to one (as $\varepsilon$ tends to 0 ).

We will prove, in Section 5, that:

THEOREM 0.2. The spatial planetary three-body problem affords, for $\varepsilon$ small enough, infinitely many periodic solutions, with larger and larger minimal period, accumulating onto each elliptic invariant torus of the family $\mathcal{F}$. 
The proof of Theorem 0.2 boils down to check that the non-resonance and non-degeneracy twist conditions of Theorem 1.1 are fulfilled for the spatial planetary three-body problem. This task is accomplished by estimates based on a careful KAM analysis.

Finally, in Section 6, we prove the existence of other periodic orbits of the spatial planetary three-body problem, the "periodic analogue" of the elliptic tori of $[\mathrm{BCV}]$. More precisely, let consider the integrable (i.e. $\varepsilon=0$ ) threebody problem when the two planets revolve around the star along circular orbits without interacting (decoupled two-body systems). Some of these motions will be periodic with, say, minimal period $T$. Taking into account the mutual interaction between the planets (i.e. $\varepsilon>0$ ) we prove the existence of, at least, two, slightly deformed, $T$-periodic orbits. The parameter $\varepsilon$ must belong to a suitable interval of values depending on the period $T$.

Theorem 0.3. There exist $T_{0}>0$ and functions $0<\underline{\varepsilon}(T)<\bar{\varepsilon}(T)$, defined for $T \in\left[T_{0}, \infty\right)$ and decreasing to zero as $T \rightarrow+\infty$ such that, for any circular periodic orbits of the decoupled three-body problem with minimal period $T \geq T_{0}$, for all $\underline{\varepsilon}(T) \leq \varepsilon \leq \bar{\varepsilon}(T)$, there exist at least two, $\varepsilon$-close, geometrically distinct $T$-periodic orbits of the spatial planetary three-body problem.

The following theorem can be seen as an $\varepsilon$-fixed version of Theorem 0.3, requiring, for fixed small values of the masses of the planets, restrictions on the period $T$.

THEOREM 0.4. There exist $\varepsilon_{1}>0$ and functions $0<\underline{T}(\varepsilon)<\bar{T}(\varepsilon)$, defined for $\varepsilon \in\left(0, \varepsilon_{1}\right]$ and tending to infinity as $\varepsilon \rightarrow 0$, such that, for all $0<\varepsilon \leq \varepsilon_{1}$ and for any circular periodic orbit of the decoupled three-body problem with minimal period $\underline{T}(\varepsilon) \leq T \leq \bar{T}(\varepsilon)$, there exist at least two, $\varepsilon$-close, geometrically distinct $T$-periodic orbits of the spatial planetary three-body problem.

A more general theorem implying Theorems 0.3 and 0.4 is given in Theorem 6.1.

The paper is organized as follows. In Section 1, we give the detailed statement of Theorem 0.1, namely Theorem 1.1, and we discuss its proof. Section 2 collects some number theoretical Lemmata used in Section 4 for defining the "non-resonant" periods $T$. Section 3 is devoted to a normal form averaging result. The proof of Theorem 1.1 is addressed in Section 4. In Section 5 , after recalling the Poincaré-Delaunay formulation of the spatial planetary three-body problem, we prove, first, a finer version of the KAM result of [BCV] and, finally, Theorem 0.2. Section 6 contains the proof of Theorems $0.3,0.4$ and 6.1 where we study the persistence, for $\varepsilon>0$, of the circular decoupled periodic motions of the planets around the star, once suitable conditions on the period and the masses of the bodies are satisfied.

Acknowledgements. We thank P. Bolle and L. Chierchia for many interesting discussions. Part of this paper was written when the last two authors were visiting SISSA in Trieste. They thanks SISSA for its kind hospitality. 
Notations: We denote by $O(\xi)$ a real analytic function whose norm is bounded by $C \xi$, for a suitable constant $C>0$ and $\forall 0<\xi \leq \xi_{0}$. Mat $(n \times n, \mathbb{R})$ (resp. $\operatorname{Mat}(n \times n, \mathbb{C}))$ is the set of $n \times n$ matrices with real (resp. complex) coefficients and $\mathbf{1}_{n}$ the $n \times n$ identity matrix. $B_{r}$ denotes the (closed) ball of radius $r$ centered at $0, B_{r}^{n}$ the closed ball of radius $r$ centered at 0 using the $|\cdot|_{2}$ norm, and $D_{\rho}^{d}$ the complex $d$-ball of radius $\rho$ centered at $0 . \sharp A$ is the cardinality of the set $A$. gcd is the greatest common divisor and $1 \mathrm{~cm}$ the least common multiple. We set $e_{i}:=(0, \ldots, 1, \ldots, 0) \in \mathbb{Z}^{n}$ the $i$-th unit vector and $|a|_{1}:=\sum_{i=1}^{n}\left|a_{i}\right|$ norm. Through the paper $C_{i}, c_{i}$, const. will denote positive constants possibly depending $n, m, \omega, \Omega, \gamma, \tau, r, s, \rho, R^{*}$, etc.

\section{1. - Periodic orbits accumulating on elliptic tori}

We now give the precise statement concerning the existence of periodic orbits close to elliptic tori (Theorem 1.1). Let consider Hamiltonians of the form

(1) $\mathcal{H}_{*}\left(\mathcal{I}_{*}, \varphi_{*}, Z_{*}, \bar{Z}_{*}\right)=\omega \cdot \mathcal{I}_{*}+\Omega Z_{*} \cdot \bar{Z}_{*}+\sum_{2|k|+|a+\bar{a}| \geq 3} R_{k, a, \bar{a}}^{*}\left(\varphi_{*}\right) \mathcal{I}_{*}^{k} Z_{*}^{a} \bar{Z}_{*}^{\bar{a}}$,

where $\left(\mathcal{I}_{*}, \varphi_{*}\right) \in \mathbb{R}^{n} \times \mathbb{T}^{n}$ are action-angle variables and $\left(Z_{*}, \bar{Z}_{*}\right) \in \mathbb{C}^{2 m}$ are called the normal (or elliptic) coordinates. The phase space $\mathbb{R}^{n} \times \mathbb{T}^{n} \times \mathbb{C}^{m} \times \mathbb{C}^{m}$ is equipped with the symplectic form ${ }^{(3)} d \mathcal{I}_{*} \wedge d \varphi_{*}+\mathrm{i} d Z_{*} \wedge d \bar{Z}_{*}$.

$\omega \in \mathbb{R}^{n}$ is the frequency vector and $\Omega:=\operatorname{diag}\left(\Omega_{1}, \ldots, \Omega_{m}\right)$ is the $m \times m$ diagonal matrix of the normal (or elliptic) frequencies. Hence $\Omega Z_{*} \cdot \bar{Z}_{*}$ denotes $\sum_{1 \leq j \leq m} \Omega_{j} Z_{* j} \bar{Z}_{* j}$. We will often identify the diagonal matrix $\Omega$ with the vector $\left(\Omega_{1}, \ldots, \Omega_{m}\right) \in \mathbb{R}^{m}$. For the multi-indexes $k \in \mathbb{N}^{n}, a, \bar{a} \in \mathbb{N}^{m}$ we define $|k|:=\sum_{i=1}^{n} k_{i},|a|:=\sum_{j=1}^{m} a_{j},|\bar{a}|:=\sum_{j=1}^{m} \bar{a}_{j}$.

The Hamiltonian $\mathcal{H}_{*}$ is assumed to be real analytic for $\left(\mathcal{I}_{*}, \varphi_{*}, Z_{*}, \bar{Z}_{*}\right) \in$ $D_{r_{*}}^{n} \times \mathbb{T}_{s_{*}}^{n} \times D_{\rho_{*}}^{2 m} \subset \mathbb{C}^{2 n+2 m}$ for some positive constants $r_{*}, s_{*}, \rho_{*} \cdot{ }^{(4)}$ Real analytic means that $\mathcal{H}_{*}$ is real analytic in the real and imaginary parts of $Z_{*}$. The functions $R_{k, a, \bar{a}}^{*}\left(\varphi_{*}\right)$ can be expanded in Fourier series as

$$
R_{k, a, \bar{a}}^{*}\left(\varphi_{*}\right)=\sum_{\ell \in \mathbb{Z}^{n}} R_{k, a, \bar{a}, \ell}^{*} e^{\mathrm{i} \ell \cdot \varphi_{*}} .
$$

Since $\mathcal{H}_{*}\left(\mathcal{I}_{*}, \varphi_{*}, Z_{*}, \bar{Z}_{*}\right) \in \mathbb{R}$ for all $\left(\mathcal{I}_{*}, \varphi_{*}\right) \in \mathbb{R}^{n} \times \mathbb{T}^{n}$ and for all $Z_{*} \in \mathbb{C}^{m}$, the Taylor-Fourier coefficient $R_{k, a, \bar{a}, \ell}^{*}$ satisfy

$$
\overline{R_{k a, \bar{a}, \ell}^{*}}=R_{k, \bar{a}, a,-\ell}^{*} \text {. }
$$

${ }^{(3)}$ We denote the imaginary unit by $\mathrm{i}$ (not to be confused with $i$ often used as an index).

${ }^{(4)}$ We have used the following standard notations: for a given set $A \subset \mathbb{R}^{d}, d \in \mathbb{N}^{+}$and $\delta, s>0$, we denote by $A_{\delta}^{d}:=\left\{z \in \mathbb{C}^{d} \mid \operatorname{dist}(z, A)<\delta\right\}$ and $\mathbb{T}_{s}^{d}:=\left\{z \in \mathbb{C}^{d}|| \operatorname{Im} z_{j} \mid<s, \forall 1 \leq j \leq d\right\}$. Moreover $D_{\delta}^{d}:=\left\{z \in \mathbb{C}^{d}|| z \mid<\delta\right\}$. 
The frequency vector $(\omega, \Omega)$ is assumed to satisfy the "second order Melnikov non-resonance conditions"

(4) $|\omega \cdot \ell+\Omega \cdot h| \geq \frac{\gamma}{1+|\ell|^{\tau}}, \quad \forall \ell \in \mathbb{Z}^{n}, h \in \mathbb{Z}^{m},|h| \leq 2,(\ell, h) \neq(0,0)$

for some positive constants $\gamma, \tau$. We will use such condition in order to perform the averaging procedure of Section 3.

By condition (4), the frequency $\omega$ is rationally independent ${ }^{(5)}$ (actually Diophantine), while the vector $(\omega, \Omega)$ can possess some resonance relations. In other words, some resonances between the "linear frequencies" $\omega$ and the "normal frequencies" $\Omega$ are allowed, provided they happen at a sufficiently large order, in order to have some ergodization property of the linear flow $\{(\omega, \Omega) t\}_{t \in \mathbb{R}}$ on the torus $\mathbb{T}^{n+m}$, see the comment before Lemma 2.3. Let us now describe these possible resonance relations in detail. Possibly reordering the frequencies $\Omega_{j}$, there exists $0 \leq \hat{m} \leq m$ such that ${ }^{(6)} \hat{\omega}:=\left(\omega, \Omega_{\hat{m}+1}, \ldots, \Omega_{m}\right)$ is rationally independent on $\mathbb{Z}^{\hat{n}}$, where $\hat{n}:=n+m-\hat{m}$, but $\left(\hat{\omega}, \Omega_{j}\right)$ is rationally dependent for all $1 \leq j \leq \hat{m}$ ( $\hat{m}$ is the number of resonances). This means that there exist $M_{j} \in \mathbb{N}^{+}$and $a_{j} \in \mathbb{Z}^{\hat{n}}$ such that ${ }^{(7)}$

$$
M_{j} \Omega_{j}=a_{j} \cdot \hat{\omega} \quad \text { with } \quad \operatorname{gcd}\left(a_{j 1}, \ldots, a_{j \hat{n}}, M_{j}\right)=1, \quad \forall 1 \leq j \leq \hat{m} .
$$

The Hamiltonian system $^{(8)}$

$$
\dot{\mathcal{I}}_{*}=-\partial_{\varphi_{*}} \mathcal{H}_{*}, \quad \dot{\varphi}_{*}=\partial_{\mathcal{I}_{*}} \mathcal{H}_{*}, \quad \dot{Z}_{*}=\mathrm{i} \partial_{\bar{Z}_{*}} \mathcal{H}_{*}, \quad \dot{\bar{Z}}_{*}=-\mathrm{i} \partial_{Z_{*}} \mathcal{H}_{*}
$$

possesses the elliptic invariant torus

$$
\mathcal{T}:=\left\{\left(\mathcal{I}_{*}, \varphi_{*}, Z_{*}, \bar{Z}_{*}\right) \in \mathbb{R}^{n} \times \mathbb{T}^{n} \times \mathbb{C}^{2 m} \mid \mathcal{I}_{*}=0, Z_{*}=\bar{Z}_{*}=0\right\}
$$

supporting the quasi-periodic solutions $\left(0, \varphi_{* 0}+\omega t, 0,0\right)$.

We define the symmetric "twist" matrix $\mathcal{R} \in \operatorname{Mat}(n \times n, \mathbb{R})$

$$
\begin{aligned}
\mathcal{R}_{i, i^{\prime}} & :=\left(1+\delta_{i, i^{\prime}}\right) R_{e_{i}+e_{i^{\prime}}, 0,0,0}^{*} \\
& -\sum_{\substack{1 \leq j \leq m \\
\ell \in \mathbb{Z}^{n}}} \frac{1}{\omega \cdot \ell+\Omega_{j}}\left(R_{e_{i}, e_{j}, 0, \ell}^{*} R_{e_{i^{\prime}}, 0, e_{j},-\ell}^{*}+R_{e_{i}, 0, e_{j},-\ell}^{*} R_{e_{i^{\prime}}, e_{j}, 0, \ell}^{*}\right),
\end{aligned}
$$

${ }^{(5)}$ I.e. $\omega \cdot \ell \neq 0, \forall \ell \in \mathbb{Z}^{n}$.

(6) If $\hat{m}=m$ we set $\hat{\omega}:=\omega$.

${ }^{(7)}$ We denote by gcd and $1 \mathrm{~cm}$ the greatest common divisor and the least common multiple, respectively.

${ }^{\left({ }^{8}\right)}$ Setting $Z_{*}=\left(P_{*}+\mathrm{i} Q_{*}\right) / \sqrt{2}, \bar{Z}_{*}=\left(P_{*}-\mathrm{i} Q_{*}\right) / \sqrt{2}$ with $\left(P_{*}, Q_{*}\right) \in \mathbb{R}^{2 m}$ the last two equations are equivalent to $\dot{Q}_{*}=\partial_{P_{*}} \mathcal{H}, \dot{P}_{*}=-\partial_{Q_{*}} \mathcal{H}$. 
where $R_{k, a, \bar{a}, \ell}^{*}$ are the Fourier coefficients, introduced in (2), of $R_{k, a, \bar{a}}^{*}\left(\varphi_{*}\right)$ and $\delta_{i, i^{\prime}}:=1$ if $i=i^{\prime}$ and 0 if $i \neq i^{\prime}$. We also define the matrix $\mathcal{Q} \in \operatorname{Mat}(m \times n, \mathbb{R})$ as

$$
\begin{aligned}
\mathcal{Q}_{j i} & :=R_{e_{i}, e_{j}, e_{j}, 0}^{*}-\sum_{\substack{1 \leq i^{\prime} \leq n \\
\ell \in \mathbb{Z}^{n}}} \frac{\ell_{i^{\prime}}}{\omega \cdot \ell+\Omega_{j}}\left(R_{e_{i}, e_{j}, 0, \ell}^{*} R_{e_{i^{\prime}}, 0, e_{j},-\ell}^{*}+R_{e_{i}, 0, e_{j},-\ell}^{*} R_{e_{i^{\prime}}, e_{j}, 0, \ell}^{*}\right) \\
& -\sum_{\substack{1 \leq j^{\prime} \leq m \\
\ell \in \mathbb{Z}^{n}}} \frac{1}{\omega \cdot \ell+\Omega_{j^{\prime}}}\left(R_{0, e_{j}, e_{j}+e_{j^{\prime}},-\ell}^{*} R_{e_{i}, e_{j^{\prime}}, 0, \ell}^{*}+R_{0, e_{j}+e_{j^{\prime}, e_{j}, \ell}^{*}}^{*} R_{e_{i}, 0, e_{j^{\prime}},-\ell}^{*}\right) .
\end{aligned}
$$

for $1 \leq j \leq m, 1 \leq i \leq n$. We stress that $\mathcal{R}$ and $\mathcal{Q}$ are real matrices by (3).

We now give the precise statement of Theorem 0.1.

THEOREM 1.1. Let the Hamiltonian $\mathcal{H}_{*}$ in (1) be real analytic in $D_{r_{*}}^{n} \times \mathbb{T}_{s_{*}}^{n} \times D_{\rho_{*}}^{2 m}$ and satisfy condition (4). Assume the "twist" condition $\operatorname{det} \mathcal{R} \neq 0$ and one of the following "non-resonance" conditions:

(a) one of the three cases below holds:

(i) $m=1,2 \quad$ (low number of elliptic directions),

(ii) $\hat{m}=0 \quad$ (no resonances among $(\omega, \Omega)$ ),

(iii) $M_{j} \geq \hat{m} \geq 1, \quad \forall 1 \leq j \leq \hat{m}$;

(b) $\alpha:=\min _{1 \leq j \leq m}\left|\left(\Omega-\mathcal{Q} \mathcal{R}^{-1} \omega\right)_{j}\right|>0$;

where $\hat{m}$ and $M_{j}$ are defined in (5).

Then, there exist $\eta_{0}, C_{0}, C_{1}>0^{(9)}$ such that: $\forall \eta \in\left(0, \eta_{0}\right]$, there exist an open set of periods $\mathcal{A}_{\eta} \subseteq\left[\frac{1}{\eta^{2}}, \frac{1}{\eta^{2}}+C_{0}\right]$ with measure greater than $1 / C_{0}$ such that $\forall T \in \mathcal{A}_{\eta}$ there exist $k:=k(T) \in \mathbb{Z}^{n}, \widetilde{\omega}:=\widetilde{\omega}(T) \in \mathbb{R}^{n}$, with $\widetilde{\omega} T=2 \pi k$, $|\widetilde{\omega}-\omega| \leq C_{1} \eta^{2}$, and at least $n$ geometrically distinct $T$-periodic solutions $\zeta_{\eta}(t)=\left(\mathcal{I}_{* \eta}(t), \varphi_{* \eta}(t)\right.$, $\left.Z_{* \eta}(t), \bar{Z}_{* \eta}(t)\right)$ of the Hamiltonian system (6) satisfying

(i) $\sup _{t \in \mathbb{R}}\left(\left|\mathcal{I}_{* \eta}(t)\right|+\left|Z_{* \eta}(t)\right|+\left|\bar{Z}_{* \eta}(t)\right|\right) \leq C_{1} \eta^{2}$;

(ii) $\sup _{t \in \mathbb{R}}\left|\varphi_{* \eta}(t)-\left(\varphi_{* \eta}(0)+\widetilde{\omega} t\right)\right| \leq C_{1} \eta$.

In particular, the closure of the family of periodic orbits $\zeta_{\eta}$ of (6) contains the elliptic torus $\mathcal{T}$. Moreover the minimal period $T_{\min }$ of $\zeta_{\eta}$ satisfies $T_{\min } \geq T^{1 /(\tau+1)} / C_{1}=$ $O\left(\eta^{-2 /(\tau+1)}\right)$.

IDEA OF THE PROOF OF THEOREM 1.1. Since $(\omega, \Omega)$ satisfy the second order Melnikov non-resonance conditions (4), in view of an averaging procedure (Proposition 3.1), the normal form Hamiltonian $\mathcal{H}_{*}$ is, in a suitable set of coordinates $(I, \phi, z, \bar{z}) \in \mathbb{R}^{n} \times \mathbb{T}^{n} \times \mathbb{C}^{2 m}$, and sufficiently close to the torus $\mathcal{T}$, a small perturbation of the integrable Hamiltonian

$$
H_{\text {int }}:=\omega \cdot I+\frac{\eta^{2}}{2} \mathcal{R} I \cdot I+\Omega z \bar{z}+\eta^{2} \mathcal{Q} I \cdot z \bar{z},
$$

${ }^{(9)}$ Such constants may depend on $n, m, \omega, \Omega, \gamma, \tau, r_{*}, s_{*}, \rho_{*}, R_{k, a, \bar{a}, \ell}^{*}, \hat{m}, \mathcal{Q}, \alpha$. 
where $\eta>0$ is a small rescaling parameter measuring the distance from $\mathcal{T}$. The Hamiltonian system generated by $H_{\text {int }}$ possesses the elliptic tori $\mathcal{T}\left(I_{0}\right):=\{I=$ $\left.I_{0}, \phi \in \mathbb{T}^{n}, z=0\right\}$ supporting the linear flow $t \rightarrow\left\{I_{0}, \phi_{0}+\left(\omega+\eta^{2} \mathcal{R} I_{0}\right) t, 0\right\}$. On the normal space the dynamics is described by $\dot{z}=i \Omega_{\eta}\left(I_{0}\right) z$ where $\Omega_{\eta}\left(I_{0}\right):=$ $\Omega+\eta^{2} \mathcal{Q} I_{0}$ is the vector of the "shifted elliptic frequencies". Our task is to find periodic solutions bifurcating from the ones of $H_{\text {int }}$.

The system is properly nonlinear by the "twist condition" det $\mathcal{R} \neq 0$ (see Remarks 4.3 and 4.4 for a comment). Such condition involves only finitely many Taylor coefficients $R_{k, a, \bar{a}}^{*}$ of the normal form Hamiltonian $\mathcal{H}_{*}$ and ensures, in particular, that the frequencies $\omega+\eta^{2} \mathcal{R} I_{0}$ vary with the actions $I_{0}$. When

$$
\widetilde{\omega}:=\omega+\eta^{2} \mathcal{R} I_{0} \in \frac{1}{T} 2 \pi \mathbb{Z}^{n},
$$

$\mathcal{T}\left(I_{0}\right)$ is a completely resonant torus, supporting the family of $T$-periodic motions $\mathcal{P}:=\left\{I(t)=I_{0}, \phi(t)=\phi_{0}+\widetilde{\omega} t, z(t)=0\right\}$ (completely resonant frequencies $\widetilde{\omega} \in(2 \pi / T) \mathbb{Z}^{n}$ always exists for some $T=O\left(\eta^{-2}\right)$ and $I_{0}=O(1)$, see (70), (71)). The family $\mathcal{P}$, diffeomorphic to $\mathbb{T}^{n}$, will not persist in its entirety for the complete Hamiltonian system due to resonances among the oscillations.

The key point to continue some periodic solutions of the family $\mathcal{P}$ is to choose properly the "1-dimensional parameter" $T$ (the period) and the actions $I_{0}$ : the period $T$ and the "shifted elliptic frequencies" $\Omega_{\eta}\left(I_{0}\right)$ must satisfy a suitable non-resonance property, see (69).

In Lemma 4.1 (resp. 4.2) we actually show the existence, assuming condition $(a)$ (resp. $(b)$ ) in Theorem 1.1, of "non resonant" periods $T$. Note that in condition $(a)$ the "coupling matrix" $\mathcal{Q}$ does not play any role (in particular for $m=2$ which is the case of the spatial three body problem). If conditions (a)-(ii)-(iii) are not verified, the system possesses "lower order" resonances (see equation (5)), which are an obstruction for finding "non-resonant" periods $T$. In this case one needs, in order to "move away" from the resonances, to evaluate the matrix $\mathcal{Q}$ and check condition (b). Conditions (a)-(b) are, indeed, sharp: if violated, it is not possible, in general, to find any "non-resonant" period $T$, see Remark 4.1.

After the previous construction, the proof of Theorem 1.1 is based on a Lyapunov-Schmidt reduction, variational in nature, inspired to [ACE]-[AB]. The non-resonance property (69) and the "twist condition" $\operatorname{det} \mathcal{R} \neq 0$, allow to build, by means of the Contraction Mapping Theorem, a suitable family of pseudo$T$-periodic solutions branching off the family $\mathcal{P}$, see Lemma 4.3. Finally, by a variational argument, we will select at least $n\left(=\right.$ cat $\left.\mathbb{T}^{n-1}\right)$ geometrically distinct $T$-periodic solutions from them.

Finally, we recall that, for strictly convex nearly integrable Hamiltonian systems, $[\mathrm{BK}]$ have proved, via a variational argument, the existence of periodic orbits with arbitrarily large minimal periods. We have not tried to extend their approach in the present case, since the unperturbed Hamiltonian $H_{\text {int }}$ is not-strictly convex (it is linear in the elliptic action variables $z \bar{z}$ ). Moreover the previously described phenomenon of resonances between the period $T$ and 
the "shifted elliptic frequencies" is hardly recognizable by a purely variational approach (which works well for finding periodic orbits near maximal dimensional tori and probably near hyperbolic one's).

\section{2. - Study of the resonances and technical Lemmata}

In this section we first collect some number theoretical Lemmata that will be used in section 4 for defining the "non-resonant periods" $T$ (Lemma 2.3 will be used in Lemma 4.1).

LeMma 2.1. Let $a_{1}, \ldots, a_{n} \in \mathbb{Z}, M \in \mathbb{N}$ with $\operatorname{gcd}\left(a_{1}, \ldots, a_{n}, M\right)=1$. Then, $\forall b \in \mathbb{Z}$, the congruence $a_{1} k_{1}+\cdots+a_{n} k_{n} \equiv b \bmod M$ has $M^{n-1}$ solutions.

Proof. Recall a well known result from number theory: let $a, b, M \in \mathbb{Z}$ and $c:=\operatorname{gcd}(a, M)$; then the congruence $a k \equiv b \bmod M, k \in \mathbb{Z}$, has solution if and only if ${ }^{(10)} c \mid b$ and in this case it has exactly $c$ solutions. It means that there exist integers $0 \leq k_{1}<\cdots<k_{c} \leq M-1$ such that $a k_{h}-b \in M \mathbb{Z} \forall 1 \leq h \leq c$, but $a k-b \notin M \mathbb{Z} \forall k \in\{0, \ldots, M-1\}, k \neq k_{h} \forall 1 \leq h \leq c$.

We now prove the Lemma by induction over $n$. The case $n=1$ is the above mentioned result. We now suppose the statement true for $n$ and prove it for $n+1$. Let $c:=\operatorname{gcd}\left(a_{n+1}, M\right)$. Our congruence is equivalent to $a_{n+1} k_{n+1} \equiv(b-$ $\left.\sum_{i=1}^{n} a_{i} k_{i}\right) \bmod M$ which has exactly $c$ solutions if and only if $c \mid\left(b-\sum_{i=1}^{n} a_{i} k_{i}\right)$. Hence we have only to prove that the number of integers $0 \leq k_{1}, \ldots, k_{n} \leq M-1$ for which $c \mid\left(b-\sum_{i=1}^{n} a_{i} k_{i}\right)$ is exactly $M^{n} / c=c^{n-1}(M / c)^{n}$. This amounts to prove that the number of integer solutions of $\sum_{i=1}^{n} a_{i} k_{i} \equiv b \bmod c$, is exactly $c^{n-1}$. This is true, by the inductive hypothesis, if $\operatorname{gcd}\left(a_{1}, \ldots, a_{n}, c\right)=1$. The last equality is actually true since, by hypothesis, $\operatorname{gcd}\left(a_{1}, \ldots, a_{n+1}, M\right)=1$; hence if $d \mid a_{1}, \ldots, a_{n}, c$, since $c \mid a_{n+1}, M$, then $d \mid a_{n+1}, M$ and it follows $d=1$.

Lemma 2.2. Let $\hat{m}, \hat{n} \in \mathbb{N}^{+}$. Let $\hat{\omega} \in \mathbb{R}^{\hat{n}}$ rationally independent. Let $\hat{\Omega} \in \mathbb{R}^{\hat{m}}$ with $M_{j} \hat{\Omega}_{j}=a_{j} \cdot \hat{\omega}$ for $M_{j} \in \mathbb{N}, a_{j} \in \mathbb{Z}^{\hat{n}}$. Suppose that $\operatorname{gcd}\left(a_{j 1}, \ldots, a_{j \hat{n}}, M_{j}\right)=1$. Let $M:=\operatorname{lcm}\left(M_{1}, \ldots, M_{\hat{m}}\right)$ and $K:=\{0, \ldots, M-1\}^{\hat{n}}$. Suppose $M_{j} \geq \hat{m}$, $\forall 1 \leq j \leq \hat{m}$. Moreover, in the case $\hat{m}=1$, suppose also $M_{1} \geq 2$. Then there exists $k \in K$ such that $a_{j} \cdot k / M_{j} \notin \mathbb{Z}, \forall 1 \leq j \leq \hat{m}$.

Proof. Let $\Lambda_{j}:=\left\{k \in K\right.$ s.t. $\left.a_{j} \cdot k \in M_{j} \mathbb{Z}\right\}, \Lambda_{j}^{*}:=\left\{k \in K_{j}\right.$ s.t. $\left.a_{j} \cdot k \in M_{j} \mathbb{Z}\right\}$, where $K_{j}:=\left\{0, \ldots, M_{j}-1\right\}^{\hat{n}}$. Since $M_{j} \mid M$ we have ${ }^{(11)} \sharp \Lambda_{j}=\left(M / M_{j}\right)^{\hat{n}} \sharp \Lambda_{j}^{*}$. Using Lemma 2.1 with the substitutions $n \rightarrow \hat{n}, b \rightarrow 0, a_{i} \rightarrow a_{j i}, M \rightarrow M_{j}$ we obtain that $\sharp \Lambda_{j}^{*}=M_{j}^{\hat{n}-1}$. Hence we have $\sharp \Lambda_{j}=M^{\hat{n}} / M_{j}$. Observing that

${ }^{(10)} c \mid b$ means that there exists $n \in \mathbb{Z}$ such that $b=c n$.

${ }^{(11)}$ We denote by $\sharp A$ the cardinality of the set $A$. 
$\{0\} \in \Lambda_{j} \forall 1 \leq j \leq \hat{m}$, we have

(9) $\sharp \bigcup_{j=1}^{\hat{m}}\left(\Lambda_{j} \backslash\{0\}\right) \leq \sum_{j=1}^{\hat{m}} \sharp\left(\Lambda_{j} \backslash\{0\}\right)=\sum_{j=1}^{\hat{m}}\left(\frac{M^{\hat{n}}}{M_{j}}-1\right)=-\hat{m}+M^{\hat{n}} \sum_{j=1}^{\hat{m}} \frac{1}{M_{j}}$.

If $\hat{m} \geq 2$, since $M_{j} \geq \hat{m}$ by hypothesis, then $-\hat{m}+M^{\hat{n}} \sum_{j=1}^{\hat{m}} \frac{1}{M_{j}} \leq-\hat{m}+M^{\hat{n}}<$ $-1+M^{\hat{n}}$. If $\hat{m}=1$, since $M_{1}=M \geq 2$ by hypothesis, $-\hat{m}+M^{\hat{n}} \sum_{j=1}^{\hat{m}} \frac{1}{M_{j}}=$ $-1+M^{\hat{n}-1}<-1+M^{\hat{n}}$. In both cases, from (9), we get

$$
\sharp \bigcup_{j=1}^{\hat{m}}\left(\Lambda_{j} \backslash\{0\}\right)<M^{\hat{n}}-1=\sharp(K \backslash\{0\})
$$

and the conclusion of the Lemma follows from $\sharp \cup_{j=1}^{\hat{m}} \Lambda_{j}<\sharp K$.

In view of the next Lemma we recall the definition of the ergodization time of a torus with linear flow. For any vector $\xi \in \mathbb{R}^{\hat{n}}$ the ergodization time $T_{\operatorname{erg}}(\xi, \varepsilon)$ required to fill $\mathbb{T}^{\hat{n}}$ within $\varepsilon>0$ is defined as (10)

$T_{\text {erg }}(\xi, \varepsilon):=\inf \left\{t \in \mathbb{R}_{+} \mid \forall x \in \mathbb{R}^{\hat{n}}, \forall 1 \leq i \leq \hat{n}, \operatorname{dist}\left(x_{i}, A_{i}+[0, t] \xi_{i}+\mathbb{Z}\right) \leq \varepsilon\right\}$

where $A$ is some point of $\mathbb{R}^{\hat{n}}$. $T_{\text {erg }}(\xi, \varepsilon)$ is clearly independent of the choice of $A$.

If $(\omega, \Omega)$ are rationally independent or are resonant only at a sufficiently high order, namely if $(\omega, \Omega) \cdot p \neq 0, \forall p \in \mathbb{Z}^{n+m}$ with $0<|p| \leq a / \delta$ for some constant $a:=a_{n, m}$, then the trajectories of the linear flow $\{(\omega, \Omega) t\}_{t \in \mathbb{R}}$ will make an arbitrarily fine $\delta$-net of $\mathbb{T}^{n+m}$, see Theorem 4.1 of [BBB]. This nonresonance assumption on $(\omega, \Omega)$ is clearly sufficient to prove (13)-(14) below. In the present case, however, the weaker non-resonance assumptions (11), (12) are sufficient.

Lemma 2.3. Let $M_{j}, a_{j}, \hat{m}$ as in (5) and suppose that

$$
M_{j} \geq \hat{m} \quad \forall 1 \leq j \leq \hat{m} .
$$

In the case $\hat{m}=1=M_{1}$ suppose also

$$
\exists n+1 \leq i_{*} \leq \hat{n}=n+m-1 \quad \text { such that } \quad a_{1 i_{*}} \neq 0 .
$$

$\forall 0<\delta \leq 1 /(2 \beta)$, where $\beta:=2 \max _{1 \leq j \leq \hat{m}}\left|a_{j}\right|_{1}$, and $\forall t_{0}>0$, there exists $\tau \geq t_{0}$ such that

(13) $\operatorname{dist}\left(\omega_{i} \tau, \mathbb{Z}\right) \leq \delta$

$$
\forall 1 \leq i \leq n,
$$

(14) $\operatorname{dist}\left(\Omega_{j} \tau, \mathbb{Z}\right) \geq \min \left\{\frac{1}{2 \beta}, \frac{1}{4 \max _{1 \leq j \leq m} M_{j}}\right\}=: d_{0}$

$\forall 1 \leq j \leq m$.

Moreover $\tau-t_{0} \leq T_{\operatorname{erg}}(\hat{\omega} / M, \delta / M)$ where $M:=\operatorname{lcm}\left(M_{1}, \ldots, M_{\hat{m}}\right)$. If $(\omega, \Omega)$ are rationally independent, namely $\hat{m}=0,(13)-(14)$ are still verified setting $\beta:=2, d_{0}:=1 / 4, M:=1$. 
Proof. We first consider the cases $\hat{m} \geq 2$ or $\hat{m}=1<M_{1}$. We are in the hypotheses of Lemma 2.2. Hence there exists $k \in K:=\{0, \ldots, M-1\}^{\hat{n}}$ such that $a_{j} \cdot k / M_{j} \notin \mathbb{Z}, \forall 1 \leq j \leq \hat{m}$. Let $^{(12)}$

$$
x:=\left\langle\frac{1}{M}\left(k+\frac{1}{\beta} \sum_{i=n+1}^{\hat{n}} e_{i}\right)-\frac{\hat{\omega}}{M} t_{0}\right\rangle \in[-1 / 2,1 / 2)^{\hat{n}} .
$$

Since $\hat{\omega} / M$ is rationally independent, its linear flow ergodizes the torus $\mathbb{R}^{\hat{n}} / \mathbb{Z}^{\hat{n}}$. Let $t_{0} \leq \tau \leq t_{0}+T_{\operatorname{erg}}(\hat{\omega} / M, \delta / M)$ the first instant for which $\exists h \in \mathbb{Z}^{\hat{n}}$ with $\left|\frac{\hat{\omega}}{M}\left(\tau-t_{0}\right)-x-h\right|_{\infty} \leq \delta / M$. If $y:=\hat{\omega} \tau-k-\frac{1}{\beta} \sum_{i=n+1}^{\hat{n}} e_{i}-M h^{\prime}$ then, by a suitable choice of $h^{\prime} \in \mathbb{Z}^{\hat{n}}$, we obtain by the construction above that $|y|_{\infty} \leq \delta$. Hence, by definition of $\hat{\omega}, \forall 1 \leq i \leq n, \omega_{i} \tau=\hat{\omega}_{i} \tau=y_{i}+k_{i}-M h_{i}^{\prime}$ and (13) holds.

Moreover, from the resonance relation (5), $\forall 1 \leq j \leq \hat{m}$,

$$
\Omega_{j} \tau=\frac{a_{j} \cdot \hat{\omega} \tau}{M_{j}}=\frac{a_{j} \cdot y}{M_{j}}+\frac{a_{j} \cdot k}{M_{j}}+\frac{1}{\beta M_{j}} \sum_{i=n+1}^{\hat{n}} a_{j i}+\frac{M}{M_{j}} a_{j} \cdot h^{\prime} .
$$

We observe that $a_{j} \cdot k / M_{j} \notin \mathbb{Z}$ implies $\operatorname{dist}\left(a_{j} \cdot k / M_{j}, \mathbb{Z}\right) \geq 1 / M_{j}$. Also, $\left|a_{j} \cdot y\right| \leq$ $\left|a_{j}\right|_{1} \delta,\left|\sum_{i=n+1}^{\hat{n}} a_{j i}\right| \leq\left|a_{j}\right|_{1}$. Hence, collecting these observations and (15), we have

$$
\operatorname{dist}\left(\Omega_{j} \tau, \mathbb{Z}\right) \geq \frac{1}{M_{j}}-\frac{\left|a_{j}\right|_{1} \delta}{M_{j}}-\frac{\left|a_{j}\right|_{1}}{\beta M_{j}} \geq \frac{1}{4 M_{j}} \quad \forall 1 \leq j \leq \hat{m}
$$

recalling also that $0<\delta \leq(1 / 2 \beta)$ and the definition of $\beta$. Finally, by definition of $\hat{\omega}$, for $\hat{m}+1 \leq j \leq m$, we have $\Omega_{j} \tau=\hat{\omega}_{n+j-\hat{m}} \tau=y_{n+j-\hat{m}}+k_{n+j-\hat{m}}+1 / \beta+$ $M h_{n+j-\hat{m}}^{\prime}$, which implies

$$
\operatorname{dist}\left(\Omega_{j} \tau, \mathbb{Z}\right) \geq \frac{1}{\beta}-\delta \geq \frac{1}{2 \beta} \quad \forall \hat{m}+1 \leq j \leq m
$$

and (14) follows from (16)-(17).

We now consider the case $\hat{m}=1, M_{1}=1$. We have $M=M_{1}=1$. We set $a:=a_{1}$ to simplify the notation. Let

$$
x:=\left\langle\sum_{i=n+1}^{\hat{n}} b_{i} e_{i}-\hat{\omega} t_{0}\right\rangle
$$

${ }^{(12)}$ We define $\left\langle\left(y_{1}, \ldots, y_{n}\right)\right\rangle:=\left(\left\langle y_{1}\right\rangle, \ldots,\left\langle y_{n}\right\rangle\right)$ where the function $\langle\cdot\rangle: \mathbb{R} \rightarrow[-1 / 2,1 / 2)$ is defined as $\langle y\rangle:=y$ for $y \in[-1 / 2,1 / 2)$ and it is 1 -periodically extended for $y \in \mathbb{R}$. Note that, for $y \neq(1 / 2)+\mathbb{Z},\langle y\rangle=y-[y]$ where $[y]$ is the closest integer to $y$. 
where

$$
b_{i}:= \begin{cases}\frac{\operatorname{sign}\left(a_{i}\right)}{2 \sum_{i=n+1}^{\hat{n}}\left|a_{i}\right|} & \text { if } \quad a_{i} \neq 0, \\ \frac{1}{2|a|_{1}} & \text { if } \quad a_{i}=0 .\end{cases}
$$

Note that $\left|b_{i}\right| \geq 1 /\left(2|a|_{1}\right)$ for all $i$. Proceeding exactly as above we find $t_{0} \leq \tau \leq t_{0}+T_{\operatorname{erg}}(\hat{\omega}, \delta)$ and an $h^{\prime} \in \mathbb{Z}^{\hat{n}}$ such that, defining

$$
y:=\hat{\omega} \tau-\sum_{i=n+1}^{\hat{n}} b_{i} e_{i}-h^{\prime}
$$

we have $|y|_{\infty} \leq \delta$ and (13) holds. The proof of (14) is slightly different. For $j=1$ we have

$$
\Omega_{1} \tau=a \cdot \hat{\omega} \tau=a \cdot y+a \cdot \sum_{i=n+1}^{\hat{n}} b_{i} e_{i}+a \cdot h^{\prime}=a \cdot y+\frac{1}{2}+a \cdot h^{\prime}
$$

where, in the last equality we have used (12) and the definition of $b_{i}$. Since $a \cdot h^{\prime} \in \mathbb{Z}$ and $|a \cdot y| \leq|a|_{1} \delta \leq 1 / 4$ we have

$$
\operatorname{dist}\left(\Omega_{1} \tau, \mathbb{Z}\right) \geq \frac{1}{2}-\frac{1}{4}=\frac{1}{4}
$$

Instead, for $2 \leq j \leq m$ we have $\Omega_{j} \tau=\hat{\omega}_{n+j-1} \tau=y_{n+j-1}+b_{n+j-1}+h_{n+j-1}^{\prime}$ which implies

(19) $\operatorname{dist}\left(\Omega_{j} \tau, \mathbb{Z}\right) \geq b_{n+j-1}-\delta \geq \frac{1}{2|a|_{1}}-\delta=\frac{1}{\beta}-\delta \geq \frac{1}{2 \beta} \quad \forall 2 \leq j \leq m$,

and (14) follows from (18)-(19).

We finally consider the case $\hat{m}=0$, which is the simplest one since the linear flow of $\hat{\omega}=(\omega, \Omega)$ ergodizes the whole torus $\mathbb{T}^{n+m}$. Let define

$$
x:=\left\langle\frac{1}{2} \sum_{i=n+1}^{n+m} e_{i}-\hat{\omega} t_{0}\right\rangle .
$$

There exists $t_{0}<\tau<t_{0}+T_{\operatorname{erg}}(\hat{\omega}, \delta)$ and $h \in \mathbb{Z}^{n+m}$ such that $\left|\hat{\omega}\left(\tau-t_{0}\right)-x-h\right|_{\infty} \leq$ $\delta$, with $0<\delta \leq 1 / 4$. Arguing as before we get $\left|\hat{\omega} \tau-(1 / 2) \sum_{i=n+1}^{n+m} e_{i}-h^{\prime}\right|_{\infty} \leq \delta$ for a suitable $h^{\prime} \in \mathbb{Z}^{n+m}$ and the estimates (13)-(14) follow. The Lemma is proved.

The next two Lemmata, whose proof is omitted, will be used in the proofs of Theorems 1.1 and 0.2 , for constructing the pseudo periodic solutions through the Contraction Mapping Theorem. 
Lemma 2.4. Let $\left(X,|\cdot|_{X}\right)$ and $\left(Y,|\cdot|_{Y}\right)$ be Banach spaces, $L: Y \longrightarrow X$ be a linear bounded operator and $P: X \longrightarrow Y$ be a $C^{1}$ map. Assume that

$$
\delta_{0} \geq 2|L(P(0))|_{X}
$$

and

$$
\sup _{x \in B_{\delta_{0}}}|D P(x)| \leq \frac{1}{2|L|} .
$$

Then, there exists a unique $x^{\star} \in B_{\delta_{0}}$ such that $x^{\star}=L\left(P\left(x^{\star}\right)\right)$.

The next Lemma defines a suitable "Green operator" $L$ associated to the linear system (26) below.

Lemma 2.5. Let $T>0, \Omega \in \operatorname{Mat}(m \times m, \mathbb{R}), M \in \operatorname{Mat}(n \times n, \mathbb{R})$ and define $\mathcal{M}:=\mathbf{1}_{m}-e^{\mathrm{i} \Omega T} \in \operatorname{Mat}(m \times m, \mathbb{C})$. Assume that $M$ and $\mathcal{M}$ are invertible. Let

$$
\begin{aligned}
& Y:=C\left([0, T], \mathbb{R}^{n} \times \mathbb{T}^{n} \times \mathbb{C}^{m}\right) \\
& X:=\{(J, \psi, z) \in Y \text { s.t. } \psi(0)=\psi(T)=0, z(0)=z(T)\} .
\end{aligned}
$$

$X$ and $Y$, endowed with the sup-norm $\|(\hat{J}, \hat{\psi}, \hat{z})\|:=\sup _{t \in[0, T]}(|\hat{J}(t)|,|\hat{\psi}(t)|$, $|\hat{z}(t)|)$, are Banach spaces.

For any $(\hat{J}, \hat{\psi}, \hat{z}) \in Y$ define the constants

$$
\begin{aligned}
\alpha(\hat{J}, \hat{\psi}) & :=-\frac{1}{T}\left(\int_{0}^{T} \int_{0}^{s} \hat{J}(\theta) d \theta d s+M^{-1} \int_{0}^{T} \hat{\psi}(\theta) d \theta\right) \in \mathbb{R}^{n}, \\
\beta(\hat{z}) & :=\mathcal{M}^{-1} e^{\mathrm{i} \Omega T} \int_{0}^{T} e^{-\mathrm{i} \Omega \theta} \hat{z}(\theta) d \theta \in \mathbb{C}^{m}
\end{aligned}
$$

and the linear "Green" operator $L: Y \longrightarrow X$ by

$$
L\left(\begin{array}{c}
\hat{J} \\
\hat{\psi} \\
\hat{z}
\end{array}\right):=\left(\begin{array}{c}
\alpha(\hat{J}, \hat{\psi})+\int_{0}^{t} \hat{J}(s) d s \\
M \alpha t+M \int_{0}^{t} \int_{0}^{s} \hat{J}(\theta) d \theta d s+\int_{0}^{t} \hat{\psi}(\theta) d \theta \\
e^{\mathrm{i} \Omega t}\left(\beta(\hat{z})+\int_{0}^{t} e^{-\mathrm{i} \Omega \theta} \hat{z}(\theta) d \theta\right)
\end{array}\right) .
$$

The Green operator L satisfies

$$
|L| \leq C\left(\left|M^{-1}\right|+|M| T^{2}+|M|\left|M^{-1}\right| T+\left|\mathcal{M}^{-1}\right| T\right)
$$

for a suitable constant $C>0$ depending on $\sup _{t \in[0, T]}\left|e^{\mathrm{i} \Omega t}\right|, n$ and $m$.

Setting $(J, \psi, z)=L(\hat{J}, \hat{\psi}, \hat{z})$, then $(J, \psi, z) \in C^{1}$ and

$$
\left\{\begin{array}{l}
\dot{J}=\hat{J}, \\
\dot{\psi}-M J=\hat{\psi}, \\
\dot{z}-\mathrm{i} \Omega z=\hat{z} .
\end{array}\right.
$$

The straightforward proof is omitted. We only point out that $L(Y) \subseteq X$ by definition of the constants $\alpha(\hat{J}, \hat{\psi})$ and $\beta(\hat{z})$. 


\section{3. - Normal form around an elliptic torus}

In order to study the dynamics of the Hamiltonian system (6) in a small neighborhood of $\mathcal{T}$ it is a convenient device to perform the following rescaling

$$
\mathcal{I}_{*}:=\eta^{2} \mathcal{I}, \quad \varphi_{*}:=\varphi, \quad Z_{*}:=\eta Z, \quad \bar{Z}_{*}:=\eta \bar{Z},
$$

where $\eta>0$ is a positive small parameter. Note that the linear transformation (27) preserves the torus $\mathcal{T}$ and that a domain of order one in the new variables $(\mathcal{I}, \varphi, Z, \bar{Z})$ correspond to a domain in the old variables $\left(\mathcal{I}_{*}, \varphi_{*}, Z_{*}, \bar{Z}_{*}\right)$ that shrinks towards $\mathcal{T}$ for $\eta$ tending to zero.

The new Hamiltonian $\mathcal{H}(\mathcal{I}, \varphi, Z, \bar{Z})=\eta^{-2} \mathcal{H}_{*}\left(\eta^{2} \mathcal{I}, \varphi, \eta Z, \eta \bar{Z}\right)$ writes

$$
\text { (28) } \mathcal{H}(\mathcal{I}, \varphi, Z, \bar{Z})=\omega \cdot \mathcal{I}+\Omega Z \cdot \bar{Z}+\sum_{2|k|+|a+\bar{a}| \geq 3} \eta^{2|k|+|a+\bar{a}|-2} R_{k, a, \bar{a}}^{*}(\varphi) \mathcal{I}^{k} Z^{a} \bar{Z}^{\bar{a}}
$$

and it is analytic on

$$
D_{r_{*} / \eta^{2}}^{n} \times \mathbb{T}_{s_{*}}^{n} \times D_{\rho_{*} / \eta}^{2 m} .
$$

In order to find periodic solutions with large period close to the elliptic torus $\mathcal{T}$ we will apply in Section 4 a finite dimensional reduction of Lyapunov-Schmidt type. For this purpose (see Remark 4.2) we need first to perform a symplectic change of variables which eliminates from the Hamiltonian $\mathcal{H}$ defined in (28) the following terms ${ }^{(13)}$

$$
\begin{gathered}
\eta \sum_{\substack{1 \leq i \leq n \\
|a+\bar{a}|=1}} R_{e_{i}, a, \bar{a}}^{*}(\varphi) \mathcal{I}_{i} Z^{a} \bar{Z}^{\bar{a}}, \\
\eta^{2} \sum_{\substack{|k|=2, \ell \neq 0 \\
\eta^{2}}} R_{k, 0,0, \ell}^{*} \mathcal{I}^{k} e^{\mathrm{i} \ell \cdot \varphi}, \\
\eta_{\substack{1 \leq i \leq n \\
|a+\bar{a}|=2 \\
a \neq \bar{a} \text { or } \ell \neq 0}} R_{e_{i}, a, \bar{a}, \ell}^{*} \mathcal{I}_{i} Z^{a} \bar{Z}^{\bar{a}} e^{\mathrm{i} \ell \cdot \varphi}, \\
\sum_{\substack{2|k|+|a+\bar{a}|=5 \\
|a+\bar{a}|=1}} R_{k, a, \bar{a}}^{*}(\varphi) \mathcal{I}^{k} Z^{a} \bar{Z}^{\bar{a}} .
\end{gathered}
$$

This task will be accomplished in the next proposition. The term (30) will be "averaged out" since $\omega$ is Diophantine; the terms (29) and (32) using the first order Melnikov non-resonance conditions (namely conditions (4) for $|h| \leq 1$ ), and, finally, the term (31) using the second order Melnikov non-resonance conditions (4).

${ }^{(13)}$ For short, in the sequel we will often omit the summation over $\ell \in \mathbb{Z}^{n}$. 
Proposition 3.1 (Averaging). Let the Hamiltonian $\mathcal{H}$, defined in (28), be real analytic on $D_{r}^{n} \times \mathbb{T}_{s}^{n} \times D_{\rho}^{2 m}$ and satisfy the second-order Melnikov non-resonance conditions (4). Then, for $\eta$ small enough, depending on $n, m, \omega, \Omega, \gamma, \tau, r, s, \rho$, there exists an analytic canonical change of coordinates $\Phi:(I, \phi, z, \bar{z}) \rightarrow(\mathcal{I}, \varphi, Z, \bar{Z})$, $\eta$-close to the identity,

$$
\Phi: D_{r / 2}^{n} \times \mathbb{T}_{s / 2}^{n} \times D_{\rho / 2}^{2 m} \longrightarrow D_{r}^{n} \times \mathbb{T}_{s}^{n} \times D_{\rho}^{2 m},
$$

transforming the Hamiltonian $\mathcal{H}$ into the Hamiltonian ${ }^{(14)}$

$$
\begin{aligned}
H= & \mathcal{H} \circ \Phi=\omega \cdot I+\Omega z \bar{z}+\eta \sum_{|a+\bar{a}|=3} R_{0, a, \bar{a}}^{*}(\phi) z^{a} \bar{z}^{\bar{a}} \\
& +\eta^{2}\left[\frac{1}{2} \mathcal{R} I \cdot I+\mathcal{Q} I \cdot z \bar{z}+\sum_{|a+\bar{a}|=4} R_{0, a, \bar{a}}(\phi) z^{a} \bar{z}^{\bar{a}}\right] \\
& +\eta^{3} \sum_{\substack{2|k|+|a+\bar{a}|=5 \\
|a+\bar{a}|=3,5}} R_{k, a, \bar{a}}(\phi) I^{k} z^{a} \bar{z}^{\bar{a}}+O\left(\eta^{4}\right),
\end{aligned}
$$

for suitable $R_{k, a, \bar{a}} \in \mathbb{C}, R_{k, a, \bar{a}}(\phi)$ analytic on $\mathbb{T}_{s / 2}^{n}$, and where $\mathcal{R}$ is the symmetric twist matrix defined in (7) and $\mathcal{Q} \in \operatorname{Mat}(m \times n, \mathbb{R})$ is defined in (8).

Proof. We rewrite the Hamiltonian (28) in the form

$$
\mathcal{H}(\mathcal{I}, \varphi, Z, \bar{Z})=\sum_{j \geq 0} \eta^{j} R_{*}^{(j)}(\mathcal{I}, \varphi, Z, \bar{Z})
$$

with

$$
R_{*}^{(0)}:=\omega \cdot \mathcal{I}+\Omega Z \cdot \bar{Z} \quad \text { and } \quad R_{*}^{(j)}:=\sum_{2|k|+|a+\bar{a}|=j+2} R_{k, a, \bar{a}, \ell}^{*} \mathcal{I}^{k} Z^{a} \bar{Z}^{\bar{a}} e^{\mathrm{i} \ell \cdot \varphi}
$$

where we have expanded in Fourier series the functions $R_{k, a, \bar{a}}^{*}(\varphi)$ as in (2).

We want to define a canonical change of variables $\Phi$ from the new variables $\zeta:=(I, \phi, z, \bar{z})$ to the old ones $\mathcal{Z}:=(\mathcal{I}, \varphi, Z, \bar{Z})$, as the flow at time 1 of a suitable Hamiltonian $\chi$. Precisely $\Phi:=\Phi_{\chi}^{1}$, where $\Phi_{\chi}^{t}\left(\zeta_{0}\right):=\zeta(t)$ is the unique solution of the Hamiltonian system

$$
\dot{I}(t)=-\partial_{\phi} \chi(\zeta(t)), \quad \dot{\phi}(t)=\partial_{I} \chi(\zeta(t)),
$$

$$
\dot{z}(t)=\mathrm{i} \partial_{\bar{z}} \chi(\zeta(t)), \quad \dot{\bar{z}}(t)=-\mathrm{i} \partial_{z} \chi(\zeta(t))
$$

with initial conditions $\zeta(0)=\zeta_{0}$.

${ }^{(14)}$ We denote $\mathcal{Q} I \cdot z \bar{z}:=\sum_{\substack{1 \leq j \leq m \\ 1 \leq i \leq n}} \mathcal{Q}_{j i} I_{i} z_{j} \bar{z}_{j}$. 
The Lie operator (Poisson brackets), acting on a function $g:=g(\zeta)$, is defined as

$$
L_{\chi} g:=\{g, \chi\}:=\partial_{\phi} g \partial_{I} \chi-\partial_{I} g \partial_{\phi} \chi+\mathrm{i} \partial_{z} g \partial_{\bar{z}} \chi-\mathrm{i} \partial_{\bar{z}} g \partial_{z} \chi .
$$

For every integer $j \geq 0$ we also set

$$
L_{\chi}^{0} g:=g, \quad L_{\chi}^{j} g:=L_{\chi} L_{\chi}^{j-1} g .
$$

The new Hamiltonian $H=\mathcal{H} \circ \Phi$ can be developed, for all $j_{0} \in \mathbb{N}^{+}$, as

$$
H:=\mathcal{H} \circ \Phi=\sum_{j=0}^{j_{0}} \frac{1}{j !} L_{\chi}^{j} \mathcal{H}+\frac{1}{j_{0} !} \int_{0}^{1}(1-\xi) L_{\chi}^{j_{0}+1} \mathcal{H} \circ \Phi_{\chi}^{\xi} d \xi .
$$

We look for a Hamiltonian $\chi$ of the form

$$
\begin{aligned}
& \chi=\sum_{j=1}^{j_{0}} \eta^{j} \chi^{(j)}, \quad \text { where } \\
& \chi^{(j)}:=\chi^{(j)}(I, \phi, z, \bar{z}):=\sum_{2|k|+|a+\bar{a}|=j+2} \chi_{k, a, \bar{a}, \ell} I^{k} z^{a} \bar{z}^{\bar{a}} e^{\mathrm{i} \ell \cdot \phi}
\end{aligned}
$$

will be chosen later on. For the sake of simplicity we will often omit in the notation the summation over $\ell \in \mathbb{Z}^{m}$. Inserting (40) in (39) we obtain

$$
H=\sum_{d=0}^{j_{0}} \eta^{d} R^{(d)}+\sum_{d=1}^{j_{0}} \eta^{d} \sum_{j=0}^{j_{0}} \frac{1}{j !} \sum_{h=0}^{d-1} \sum_{\substack{h+i_{1}+\cdots+i_{j}=d \\ 1 \leq i_{1}, \ldots, i_{j} \leq d}} L_{\chi^{\left(i_{1}\right)}} \ldots L_{\chi}^{\left(i_{j}\right)} R_{*}^{(h)}+O\left(\eta^{j_{0}+1}\right)
$$

Denoting $[\cdot]_{d}$ the $d$-th order in $\eta$, we obtain

$$
[H]_{1}=R_{*}^{(1)}+L_{\chi^{(1)}} R_{*}^{(0)}
$$

and

$$
[H]_{d}=R_{*}^{(d)}+L_{\chi}^{(d)} R_{*}^{(0)}+\sum_{j=0}^{j_{0}} \frac{1}{j !} \sum_{h=0}^{d-1} \sum_{\substack{h+i_{1}+\cdots+i_{j}=d \\ 1 \leq i_{1}, \ldots i_{j} \leq d-1}} L_{\chi}^{\left(i_{1}\right)} \ldots L_{\chi}^{\left(i_{j}\right)} R_{*}^{(h)}
$$

Observe that

$$
\sum_{\substack{2|k|+|a+\bar{a}|=h+2 \\ 2\left|k^{\prime}\right|+\left|a^{\prime}+\bar{a}^{\prime}\right|=h^{\prime}+2}}\left\{I^{k} z^{a} \bar{z}^{\bar{a}} e^{\mathrm{i} \ell \cdot \phi}, I^{k^{\prime}} z^{a^{\prime}} \bar{z}^{\bar{a}^{\prime}} e^{\mathrm{i} \ell^{\prime} \cdot \phi}\right\}
$$

$$
\sum_{2\left|k^{\prime \prime}\right|+\left|a^{\prime \prime}+\bar{a}^{\prime \prime}\right|=h+h^{\prime}+2} c_{k^{\prime \prime}, a^{\prime \prime}, \bar{a}^{\prime \prime}, \ell^{\prime \prime}} k^{k^{\prime \prime}} z^{a^{\prime \prime}} \bar{a}^{\bar{a}^{\prime \prime}} e^{\mathrm{i} \ell^{\prime \prime} \cdot \phi},
$$


for suitable constants $c_{k^{\prime \prime}, a^{\prime \prime}, \bar{a}^{\prime \prime}, \ell^{\prime \prime}}$, which are explicitly given by the following formula:

$$
\begin{aligned}
\left\{I^{k} z^{a} \bar{z}^{\bar{a}} e^{\mathrm{i} \ell \cdot \phi}, I^{k^{\prime}} z^{a^{\prime}} \bar{z}^{\prime} e^{\mathrm{i} \ell^{\prime} \cdot \phi}\right\} & =\mathrm{i}\left[\sum_{i=1}^{n}\left(\ell_{i} k_{i}^{\prime}-\ell_{i}^{\prime} k_{i}\right) I^{k+k^{\prime}-e_{i}} z^{a+a^{\prime}} \bar{z}^{\bar{a}+\bar{a}^{\prime}}\right. \\
& \left.+\sum_{j=1}^{m}\left(a_{j} \bar{a}_{j}^{\prime}-\bar{a}_{j} a_{j}^{\prime}\right) I^{k+k^{\prime}} z^{a+a^{\prime}-e_{j}} \bar{z}^{\bar{a}+\bar{a}^{\prime}-e_{j}}\right] e^{\mathrm{i}\left(\ell+\ell^{\prime}\right) \cdot \phi} .
\end{aligned}
$$

Thus, we obtain that

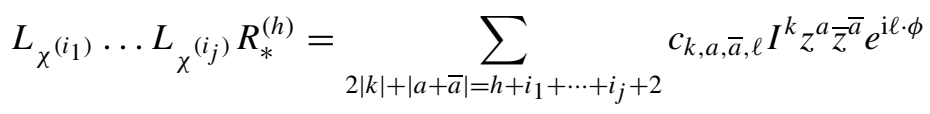

for suitable constants $c_{k, a, \bar{a}, \ell}$. Hence,

$$
[H]_{d}=L_{\chi^{(d)}} R_{*}^{(0)}+\sum_{2|k|+|a+\bar{a}|=d+2} R_{k, a, \bar{a}, \ell} I^{k} z^{a} \bar{z}^{\bar{a}} e^{\mathrm{i} \ell \cdot \phi}
$$

for suitable $R_{k, a, \bar{a}, \ell}$ with

$$
R_{k, a, \bar{a}, \ell}:=R_{k, a, \bar{a}, \ell}\left(\chi^{(1)}, \ldots, \chi^{(d-1)}, R_{*}^{(0)}, \ldots, R_{*}^{(d)}\right)
$$

we note that, by means of (42) and (48)

$$
2|k|+|a+\bar{a}|=3 \quad \Longrightarrow \quad R_{k, a, \bar{a}, \ell}=R_{k, a, \bar{a}, \ell}^{*},
$$

(recall also the setting in (35)). We also evaluate

$$
\begin{aligned}
L_{\chi^{(d)}} R_{*}^{(0)} & =\left\{\omega \cdot I+\Omega z \bar{z}, \chi^{(d)}\right\} \\
& =\sum_{2|k|+|a+\bar{a}|=d+2}-\mathrm{i}(\omega \cdot \ell+\Omega \cdot(a-\bar{a})) \chi_{k, a, \bar{a}, \ell} I^{k} z^{a} \bar{z}^{\bar{a}} e^{\mathrm{i} \ell \cdot \phi} .
\end{aligned}
$$

We define the following "resonant" set:

$$
S:=S_{1} \cup S_{2} \cup S_{3} \subset\left\{(k, a, \bar{a}, \ell) \quad \text { s.t. } \quad 3 \leq 2|k|+|a+\bar{a}| \leq 5, \quad \ell \in \mathbb{Z}^{n}\right\},
$$

with $S_{2}=S_{2}^{0} \cup S_{2}^{1} \cup S_{2}^{2}$ and

$$
\begin{aligned}
& S_{1}:=\left\{k=0, \quad|a+\bar{a}|=3, \quad \ell \in \mathbb{Z}^{n}\right\} \\
& S_{2}^{0}:=\left\{k=0, \quad|a+\bar{a}|=4, \quad \ell \in \mathbb{Z}^{n}\right\} \\
& S_{2}^{1}:=\left\{k=e_{i}, \quad a=\bar{a}=e_{j}, \quad 1 \leq i \leq n, \quad 1 \leq j \leq m, \quad \ell=0\right\} \\
& S_{2}^{2}:=\{|k|=2, \quad a=\bar{a}=0, \quad \ell=0\} \\
& S_{3}:=\left\{2|k|+|a+\bar{a}|=5, \quad|a+\bar{a}|=3,5, \quad \ell \in \mathbb{Z}^{n}\right\} .
\end{aligned}
$$


Let $j_{0}=3 . \forall 1 \leq d \leq 3, \forall(k, a, \bar{a}, \ell)$ such that $2|k|+|a+\bar{a}|=d+2, \ell \in \mathbb{Z}^{n}$, one can check, by condition (4), that

$$
(k, a, \bar{a}, \ell) \notin S \quad \Longrightarrow \quad|\omega \cdot \ell+\Omega(a-\bar{a})| \geq \frac{\gamma}{1+|\ell|^{\tau}},
$$

and hence we can define

$$
\chi_{k, a, \bar{a}, \ell}:= \begin{cases}0 & \text { if }(k, a, \bar{a}, \ell) \in S \\ -\mathrm{i}[\omega \cdot \ell+\Omega(a-\bar{a})]^{-1} R_{k, a, \bar{a}, \ell} & \text { otherwise }\end{cases}
$$

In light of this construction, using (48) and (51), we have $\mathrm{e}^{(15)}$

$$
[H]_{d}=\Pi_{S_{d}}\left(\sum_{2|k|+|a+\bar{a}|=d+2} R_{k, a, \bar{a}, \ell} I^{k} z^{a} \bar{z}^{\bar{a}} e^{\mathrm{i} \ell \cdot \phi}\right)=\sum_{(k, a, \bar{a}, \ell) \in S_{d}} R_{k, a, \bar{a}, \ell} I^{k} z^{a} \bar{z}^{\bar{a}} e^{\mathrm{i} \ell \cdot \phi}
$$

Define

$$
R_{k, a, \bar{a}}:=R_{k, a, \bar{a}, 0}, \quad R_{k, a, \bar{a}}(\phi):=\sum_{\ell \in \mathbb{Z}^{n}} R_{k, a, \bar{a}, \ell} e^{\mathrm{i} \ell \cdot \phi} .
$$

By recurrence, using (48), it is possible to evaluate the terms $R_{k, a, \bar{a}}$ explicitly.

From (43) and (50) we have

$$
[H]_{2}=\Pi_{S_{2}}\left(\frac{1}{2}\left\{\left\{R_{*}^{(0)}, \chi^{(1)}\right\}, \chi^{(1)}\right\}+\left\{R_{*}^{(1)}, \chi^{(1)}\right\}+R_{*}^{(2)}\right) .
$$

Noting that

$$
\left\{R_{*}^{(0)}, \chi^{(1)}\right\}=-R_{*}^{(1)}+\Pi_{S_{1}} R_{*}^{(1)},
$$

we have

$$
[H]_{2}=\Pi_{S_{2}}\left(\frac{1}{2}\left\{R_{*}^{(1)}+\Pi_{S_{1}} R_{*}^{(1)}, \chi^{(1)}\right\}+R_{*}^{(2)}\right) .
$$

In order to prove (33) we only need to show that

$$
\begin{aligned}
\frac{1}{2} \mathcal{R} I \cdot I & =\Pi_{S_{2}^{2}}\left[H_{2}\right]=\frac{1}{2} \Pi_{S_{2}^{2}}\left(\left\{R_{*}^{(1)}, \chi^{(1)}\right\}\right)+\Pi_{S_{2}^{2}} R_{*}^{(2)}, \\
\mathcal{Q} I \cdot z \bar{z} & =\Pi_{S_{2}^{1}}\left[H_{2}\right]=\Pi_{S_{2}^{1}}\left(\frac{1}{2}\left\{R_{*}^{(1)}+\Pi_{S_{1}} R_{*}^{(1)}, \chi^{(1)}\right\}\right)+\Pi_{S_{2}^{1}} R_{*}^{(2)} \\
& =\Pi_{S_{2}^{1}}\left(\left\{\frac{1}{2} \Pi_{S_{1}^{c}} R_{*}^{(1)}+\Pi_{S_{1}} R_{*}^{(1)}, \chi^{(1)}\right\}\right)+\Pi_{S_{2}^{1}} R_{*}^{(2)},
\end{aligned}
$$

${ }^{(15)}$ We denote by $\Pi_{\mathcal{S}}$, where $\mathcal{S} \subset \mathbb{N}^{n+2 m} \times \mathbb{Z}^{n}$, the projection on $\mathcal{S}$ i.e.

$$
\Pi_{\mathcal{S}}\left(\sum_{k, a, \bar{a}, \ell} c_{k, a, \bar{a}, \ell} I^{k} z^{a} \bar{z}^{\bar{a}} e^{\mathrm{i} \ell \cdot \phi}\right):=\sum_{(k, a, \bar{a}, \ell) \in \mathcal{S}} c_{k, a, \bar{a}, \ell} I^{k} z^{a} \bar{z}^{\bar{a}} e^{\mathrm{i} \ell \cdot \phi} .
$$


where $S_{1}^{c}:=\left\{k=e_{i},|a+\bar{a}|=1, \ell \in \mathbb{Z}^{n}, 1 \leq i \leq n\right\}$.

We first prove (56). Using (40), (54) and (46), we have

$$
\begin{aligned}
& \Pi_{S_{2}^{2}}\left[H_{2}\right]=\sum_{j=1}^{m} \sum_{\begin{array}{c}
|k|=\left|k^{\prime}\right|=1 \\
|a+\bar{a}|=\left|a^{\prime}+\bar{a}^{\prime}\right|=1 \\
a+a^{\prime}=\bar{a}+\bar{a}^{\prime}=e_{j}
\end{array}} \frac{1}{2} \cdot \frac{R_{k, a, \bar{a}, \ell}^{*} R_{k^{\prime}, a^{\prime}, \bar{a}^{\prime},-\ell}^{*}}{-\omega \cdot \ell+\Omega(\bar{a}-a)}\left(a_{j} \bar{a}_{j}^{\prime}-\bar{a}_{j} a_{j}^{\prime}\right) I^{k+k^{\prime}} \\
& +\sum_{|k|=2} R_{k, 0,0,0}^{*} I^{k} \\
& =\sum_{j=1}^{m} \sum_{\substack{1 \leq i, i^{\prime} \leq n \\
|a+\bar{a}|=\mid a^{\prime}+\bar{a}^{\prime}=1 \\
a+a^{\prime}=\bar{a}+\bar{a}^{\prime}=e_{j}}} \frac{1}{2} \cdot \frac{R_{e_{i}, a, \bar{a}, \ell}^{*} R_{e_{i^{\prime}}, a^{\prime}, \bar{a}^{\prime},-\ell}^{*}}{-\omega \cdot \ell+\Omega(\bar{a}-a)}\left(a_{j} \bar{a}_{j}^{\prime}-\bar{a}_{j} a_{j}^{\prime}\right) I_{i} I_{i^{\prime}} \\
& +\sum_{i, i^{\prime}=1}^{n} \frac{1+\delta_{i, i^{\prime}}}{2} R_{e_{i}+e_{i^{\prime}}, 0,0,0}^{*} I_{i} I_{i^{\prime}} \\
& =\frac{1}{2} \sum_{i, i^{\prime}=1}^{n} \sum_{j=1}^{m}\left(\frac{R_{e_{i}, e_{j}, 0, \ell}^{*} R_{e_{i^{\prime}}, 0, e_{j},-\ell}^{*}}{-\omega \cdot \ell-\Omega_{j}}-\frac{R_{e_{i}, 0, e_{j}, \ell}^{*} R_{e_{i^{\prime}}, e_{j}, 0,-\ell}^{*}}{-\omega \cdot \ell+\Omega_{j}}\right) \\
& +\sum_{i, i^{\prime}=1}^{n} \frac{1+\delta_{i, i^{\prime}}}{2} R_{e_{i}+e_{i^{\prime}}, 0,0,0}^{*} I_{i} I_{i^{\prime}}=\frac{1}{2} \mathcal{R} I \cdot I,
\end{aligned}
$$

where $\delta_{i, i^{\prime}}=1$ if $i=i^{\prime}$ and 0 if $i \neq i^{\prime}$. Here, we observe that, since $|a+\bar{a}|=\left|a^{\prime}+\bar{a}^{\prime}\right|=1$, if $a+a^{\prime}=\bar{a}+\bar{a}^{\prime}=e_{j}$, then $\left(a, \bar{a}, a^{\prime}, \bar{a}^{\prime}\right)=\left(e_{j}, 0,0, e_{j}\right)$ or $\left(0, e_{j}, e_{j}, 0\right)$. So (56) directly follows from (58).

We now prove (57). Using again (40), (54) and (46), we have

$$
\begin{aligned}
& \Pi_{S_{2}^{1}}\left[H_{2}\right]=-\mathrm{i} \Pi_{S_{2}^{1}}\left(\left\{\frac{1}{2} \Pi_{S_{1}^{c}} R_{*}^{(1)}+\Pi_{S_{1}} R_{*}^{(1)},\right.\right. \\
& \left.\left.\sum_{\substack{1 \leq i^{\prime} \leq n \\
1 \leq j^{\prime} \leq m}}\left(\frac{R_{e_{i^{\prime}}, e_{j^{\prime}}, 0, \ell^{\prime}}^{*}}{\omega \cdot \ell^{\prime}+\Omega_{j^{\prime}}} z_{j^{\prime}}+\frac{R_{e_{i^{\prime}}, 0, e_{j^{\prime}}, \ell^{\prime}}^{*}}{\omega \cdot \ell^{\prime}-\Omega_{j^{\prime}}} \bar{z}_{j^{\prime}}\right) I_{i^{\prime}} e^{\mathrm{i} \ell^{\prime} \cdot \phi}\right\}\right)+\Pi_{S_{2}^{1}} R_{*}^{(2)}
\end{aligned}
$$

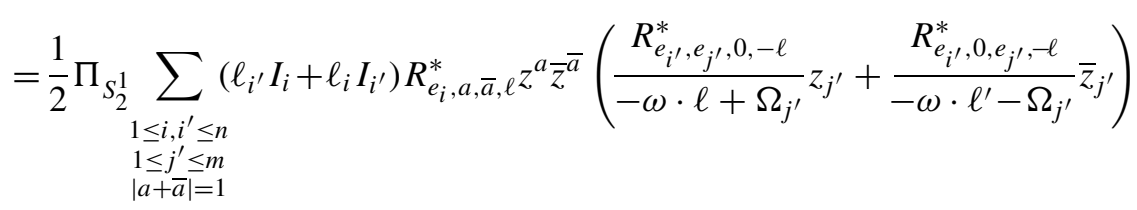




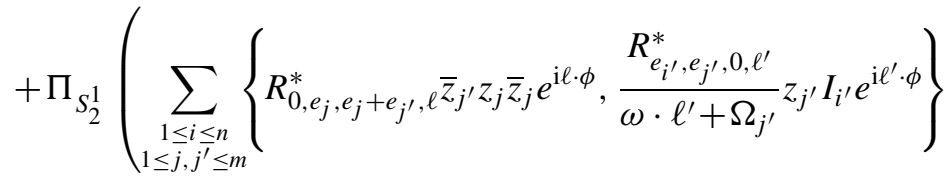

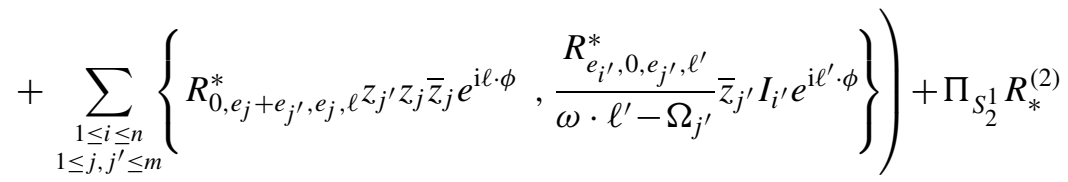

$$
\begin{aligned}
& \text { (59) }=\frac{1}{2} \sum_{\substack{1 \leq i, i^{\prime} \leq n \\
1 \leq j \leq m}} \ell_{i^{\prime}}\left(\frac{R_{e_{i}, 0, e_{j}, \ell}^{*} R_{e_{i^{\prime}}, e_{j}, 0,-\ell}^{*}}{-\omega \cdot \ell+\Omega_{j}}+\frac{R_{e_{i}, e_{j}, 0, \ell}^{*} R_{e_{i^{\prime}}, 0, e_{j},-\ell}^{*}}{-\omega \cdot \ell-\Omega_{j}}\right. \\
& \left.+\frac{R_{e_{i}, 0, e_{j}, \ell}^{*} R_{e_{i^{\prime}}, e_{j}, 0,-\ell}^{*}}{-\omega \cdot \ell+\Omega_{j}}+\frac{R_{e_{i}, e_{j}, 0, \ell}^{*} R_{e_{i^{\prime}}, 0, e_{j},-\ell}^{*}}{-\omega \cdot \ell-\Omega_{j}}\right) I_{i} z_{j} \bar{z}_{j} \\
& -\sum_{\substack{1 \leq i \leq n \\
1 \leq j, j^{\prime} \leq m}} \frac{1}{\omega \cdot \ell+\Omega_{j^{\prime}}}\left(R_{0, e_{j}, e_{j}+e_{j^{\prime}},-\ell}^{*} R_{e_{i}, e_{j^{\prime}}, 0, \ell}^{*}+R_{0, e_{j}+e_{j^{\prime}}, e_{j} \ell}^{*} R_{e_{i}, 0, e_{j^{\prime}},-\ell}^{*}\right) I_{i} z_{j} \bar{z}_{j} \\
& +\Pi_{S_{2}^{1}} \sum_{2|k|+|a+\bar{a}|=4} R_{k, a, \bar{a}, \ell}^{*} I^{k} z^{a} \bar{z}^{\bar{a}} e^{\mathrm{i} \ell \cdot \phi} .
\end{aligned}
$$

So (57) directly follows from (59).

The estimate on the new analyticity radii follows from (53) and from the fact that $\chi=O(\eta)$ (see (40)).

\section{4. - Periodic orbits winding along the torus}

In this section we prove the existence of periodic solutions of longer and longer (minimal) period shrinking closer and closer to the elliptic torus $\mathcal{T}$ (Theorem 1.1).

By Proposition 3.1 the Hamiltonian (28) can be transformed, thanks to the second order Melnikov non-resonance conditions (4), into the Hamiltonian

$$
\begin{aligned}
H(I, \phi, z, \bar{z})= & \omega \cdot I+\Omega z \bar{z}+\eta \sum_{|a+\bar{a}|=3} R_{0, a, \bar{a}}^{*}(\phi) z^{a} \bar{z}^{\bar{a}} \\
& +\eta^{2}\left[\frac{1}{2} \mathcal{R} I \cdot I+\mathcal{Q} I \cdot z \bar{z}+\sum_{|a+\bar{a}|=4} R_{0, a, \bar{a}}(\phi) z^{a} \bar{z}^{\bar{a}}\right] \\
& +\eta^{3} \sum_{\substack{2|k|+|a+\bar{a}|=5 \\
|a+\bar{a}|=3,5}} R_{k, a, \bar{a}}(\phi) I^{k} z^{a} \bar{z}^{\bar{a}}+O\left(\eta^{4}\right),
\end{aligned}
$$


analytic on

$$
D_{r_{*} /\left(2 \eta^{2}\right)}^{n} \times \mathbb{T}_{s_{* / 2}}^{n} \times D_{\rho_{*} /(2 \eta)}^{2 m} .
$$

The Hamilton's equations of motion induced by the Hamiltonian (60)

$$
\dot{I}=-\partial_{\phi} H, \quad \dot{\phi}=\partial_{I} H, \quad \dot{z}=\mathrm{i} \partial_{\bar{z}} H, \quad \dot{\bar{z}}=-\mathrm{i} \partial_{z} H,
$$

can be written as

$$
\begin{aligned}
& \dot{I}=-\eta \sum_{|a+\bar{a}|=3} \partial_{\phi} R_{0, a, \bar{a}}^{*}(\phi) z^{a} \bar{z}^{\bar{a}}-\eta^{2} \sum_{|a+\bar{a}|=4} \partial_{\phi} R_{0, a, \bar{a}}(\phi) z^{a} \bar{z}^{\bar{a}} \\
& -\eta^{3} \sum_{\substack{2|k|+|a+\bar{a}|=5 \\
|a+\bar{a}|=3,5}} \partial_{\phi} R_{k, a, \bar{a}}(\phi) I^{k} z^{a} \bar{z}^{\bar{a}}+O\left(\eta^{4}\right) \\
& \dot{\phi}_{i}=\omega_{i}+\eta^{2}\left[(\mathcal{R} I)_{i}+\sum_{1 \leq j \leq m} \mathcal{Q}_{j i} z_{j} \bar{z}_{j}\right] \\
& +\eta^{3} \sum_{\substack{2|k|+|a+\bar{a}|=5 \\
|a+\bar{a}|=3,5}} R_{k, a, \bar{a}}(\phi) k_{i} I^{k-e_{i}} z^{a} \bar{z}^{\bar{a}}+O\left(\eta^{4}\right), \\
& \dot{z}_{j}=\mathrm{i} \Omega_{j} z_{j}+\mathrm{i} \eta \sum_{|a+\bar{a}|=3} R_{0, a, \bar{a}}^{*}(\phi) \bar{a}_{j} z^{a} \bar{z}^{\bar{a}-e_{j}} \\
& +\mathrm{i} \eta^{2}\left[(\mathcal{Q} I)_{j} z_{j}+\sum_{|a+\bar{a}|=4} R_{0, a, \bar{a}}(\phi) \bar{a}_{j} z^{a} \bar{z}^{\bar{a}-e_{j}}\right] \\
& +\mathrm{i} \eta^{3} \sum_{\substack{2|k|+|a+\bar{a}|=5 \\
|a+\bar{a}|=3,5}} R_{k, a, \bar{a}}(\phi) I^{k} \bar{a}_{j} z^{a} \bar{z}^{\bar{a}-e_{j}}+O\left(\eta^{4}\right),
\end{aligned}
$$

for $i=1, \ldots, n, j=1, \ldots, m$.

Critical points of the Hamiltonian action functional

(63) $\mathcal{A}(I(t), \phi(t), z(t), \bar{z}(t)):=\int_{0}^{T} I \cdot \dot{\phi}+\mathrm{i} z \dot{\bar{z}}-H(I(t), \phi(t), z(t), \bar{z}(t)) d t$,

in a suitable space of $T$-periodic functions, are $T$-periodic solutions of (61).

\section{1. - The pseudo periodic solutions}

We will find periodic solutions of the Hamiltonian system (61) close to periodic solutions of the integrable Hamiltonian

$$
H_{i n t}:=\omega \cdot I+\frac{\eta^{2}}{2} \mathcal{R} I \cdot I+\Omega z \bar{z}+\eta^{2} \mathcal{Q} I \cdot z \bar{z}
$$


The manifold $\{z=0\}$ is invariant for the Hamiltonian system generated by $H_{\text {int }}{ }^{(16)}$

$$
\dot{I}=0, \quad \dot{\phi}=\omega+\eta^{2}\left(\mathcal{R} I+\mathcal{Q}^{T} z \bar{z}\right), \quad \dot{z}=\mathrm{i}\left(\Omega+\eta^{2} \mathcal{Q} I\right) z
$$

and it is completely filled up by the invariant tori $\mathcal{T}\left(I_{0}\right):=\left\{I=I_{0}, \phi \in\right.$ $\left.\mathbb{T}^{n}, \quad z=0\right\}$. On $\mathcal{T}\left(I_{0}\right)$ the flow is $t \rightarrow\left\{I_{0}, \phi_{0}+\left(\omega+\eta^{2} \mathcal{R} I_{0}\right) t, 0\right\}$ and in its normal space it is determined by $\dot{z}=\mathrm{i} \Omega_{\eta}\left(I_{0}\right) z$ where $\Omega_{\eta}\left(I_{0}\right)$ is the $m \times m$ diagonal matrix with real coefficients associated to the vector of the "shifted elliptic frequencies"

$$
\Omega_{\eta}\left(I_{0}\right):=\Omega+\eta^{2} \mathcal{Q} I_{0}
$$

For any $I_{0} \in \mathbb{R}^{n}, \Omega_{\eta}\left(I_{0}\right)$ is a real matrix, since $\mathcal{Q}$ is real (see page 94 ).

For suitable $T>0, I_{0} \in \mathbb{R}^{n}, k \in \mathbb{Z}^{n}$, namely when

$$
\widetilde{\omega}:=\omega+\eta^{2} \mathcal{R} I_{0}=\frac{1}{T} 2 \pi k \in \frac{1}{T} 2 \pi \mathbb{Z}^{n},
$$

the torus $\mathcal{T}\left(I_{0}\right)$ is completely resonant, supporting the family of $T$-periodic motions

$$
\mathcal{P}:=\left\{I(t)=I_{0}, \quad \phi(t)=\phi_{0}+\widetilde{\omega} t, \quad z(t)=0\right\} .
$$

The family $\mathcal{P}$ will not persist in its entirety for the complete Hamiltonian system (61). However, the non-resonance property (69) below, between the period $T$ and the "shifted elliptic frequencies" $\Omega_{\eta}\left(I_{0}\right)$, is sufficient to prove the persistence of at least $n$ geometrically distinct $T$-periodic solutions of the Hamiltonian system (61), close to $\mathcal{P}$. Precisely, the required non resonance property is

(69) $\mathcal{M}:=\mathcal{M}\left(I_{0}, T\right):=\mathbf{1}_{m}-e^{\mathrm{i} \Omega_{\eta}\left(I_{0}\right) T}$ is invertible and $\left|\mathcal{M}^{-1}\left(I_{0}, T\right)\right| \leq$ const.

Our aim is then to find $I_{0}$ and $T$ so that (67) and (69) hold: we will define $I_{0}:=I_{0}(T)$ in dependence on the "1-dimensional parameter" $T$ in such a way that (67) is identically satisfied and then we will find $T$ so that the non resonance property (69) holds. Moreover, for our perturbative arguments of Lemma 4.3, we want $I_{0}=O(1)$ and $T<2 / \eta^{2}$.

${ }^{(16)}$ We recall the usual notation for the vector $\mathcal{Q}^{T} z \bar{z} \in \mathbb{R}^{n}$ whose components are $\left(\mathcal{Q}^{T} z \bar{z}\right)_{i}:=$ $\sum_{j=1}^{m} \mathcal{Q}_{j i} I_{i} z_{j} \bar{z}_{j}, 1 \leq i \leq n$ and for the $m \times m$ diagonal matrix $\Omega+\eta^{2} \mathcal{Q} I:=\operatorname{diag}\left(\Omega_{1}+\right.$ $\left.\eta^{2}(\mathcal{Q} I)_{1}, \ldots, \Omega_{m}+\eta^{2}(\mathcal{Q} I)_{m}\right)$. 
Define, for $T \geq 1 / \eta^{2}$,

$$
\begin{aligned}
I_{0} & :=I_{0}(T):=-\frac{2 \pi}{\eta^{2} T} \mathcal{R}^{-1}\left\langle\frac{\omega T}{2 \pi}\right\rangle, \\
k & :=k(T)=\frac{\omega T}{2 \pi}-\left\langle\frac{\omega T}{2 \pi}\right\rangle,
\end{aligned}
$$

where $\left\langle\left(x_{1}, \ldots, x_{n}\right)\right\rangle:=\left(\left\langle x_{1}\right\rangle, \ldots,\left\langle x_{n}\right\rangle\right)$ and the function $\langle\cdot\rangle: \mathbb{R} \rightarrow[-1 / 2,1 / 2)$ is defined as $\langle x\rangle:=x$ for $x \in[-1 / 2,1 / 2)$ and it is 1-periodically extended for $x \in \mathbb{R}$. Notice that $I_{0} \in \mathbb{R}^{n}$ since $\mathcal{R}$ is a real matrix (see page 94). With the choice (70), (71), $\omega T+\eta^{2} \mathcal{R} I_{0}(T)=2 \pi k$, and then (67) holds. In addition, for $T \geq 1 / \eta^{2}, I_{0}(T)=O(1)$. Moreover

$$
\Omega_{\eta} T=\Omega_{\eta}\left(I_{0}(T)\right) T=\Omega T+\eta^{2} \mathcal{Q} I_{0} T=2 \pi\left(\Omega \frac{T}{2 \pi}-\mathcal{Q R}^{-1}\left\langle\omega \frac{T}{2 \pi}\right\rangle\right) .
$$

In order to prove the non-resonance property (69), we note that

$$
\left|\mathcal{M}^{-1}\right| \leq \frac{1}{\min _{1 \leq j \leq m}\left|e^{i \Omega_{\eta j} T}-1\right|} \leq \frac{1}{\min _{1 \leq j \leq m}\left|\sin \left(\Omega_{\eta j} T\right)\right|} \leq \frac{2}{\min _{1 \leq j \leq m} \operatorname{dist}\left(\Omega_{\eta j} T, 2 \pi \mathbb{Z}\right)} .
$$

Lemma 4.1. Suppose that condition (a) of Theorem 1.1 hold. Define, for $\hat{m} \geq 1$,

$$
\begin{aligned}
& d_{0}:=\min \left\{\frac{1}{4 \max _{1 \leq j \leq \hat{m}}\left|a_{j}\right|_{1}}, \frac{1}{4 \max _{1 \leq j \leq \hat{m}} M_{j}}\right\}, \\
& \delta:=\min \left\{\frac{d_{0}}{2 \max _{1 \leq j \leq m}\left|\left(\mathcal{Q R} \mathcal{R}^{-1}\right)_{j}\right|_{1}}, \frac{1}{4 \max _{1 \leq j \leq \hat{m}}\left|a_{j}\right|_{1}}\right\}
\end{aligned}
$$

where $\left(\mathcal{Q R}^{-1}\right)_{j}$ is the $j$-row of the matrix $\mathcal{Q R} \mathcal{R}^{-1}$, and, for $\hat{m}=0$,

$$
d_{0}:=\frac{1}{4}, \quad \delta:=\min \left\{\frac{1}{\left.8 \max _{1 \leq j \leq m} \mid(\mathcal{Q R})^{-1}\right)_{j}}, \frac{1}{4}\right\} .
$$

Let $M:=\operatorname{lcm}\left(M_{1}, \ldots, M_{\hat{m}}\right)$, for $\hat{m} \geq 1$, and $M=1$ for $\hat{m}=0$. Finally let ${ }^{(17)}$

$$
T_{e}:=T_{\text {erg }}\left(\frac{\hat{\omega}}{M}, \frac{\delta}{M}\right), \quad \Theta:=\min \left\{\frac{\delta}{4 \max _{1 \leq i \leq n}\left|\omega_{i}\right|}, \frac{d_{0}}{8 \max _{1 \leq j \leq m}\left|\Omega_{j}\right|}\right\} .
$$

${ }^{(17)}$ The ergodization time $T_{\text {erg }}$ was defined in (10). 
Then $\forall t_{0} \geq 0$, there exists an interval $\mathcal{J} \subset\left[t_{0}-2 \pi \Theta, t_{0}+2 \pi T_{e}+2 \pi \Theta\right]$ of length, at least $4 \pi \Theta$, such that $\forall T \in \mathcal{J}, \mathcal{M}^{-1}:=\mathcal{M}^{-1}\left(I_{0}(T), T\right)$ satisfies

$$
\left|\mathcal{M}^{-1}\right| \leq \frac{4}{\pi d_{0}}
$$

Proof. In order to define the periods $T$ we want to use Lemma 2.3. Let us verify that its hypothesis are fulfilled. If $(\omega, \Omega)$ are rationally independent, i.e. $\hat{m}=0$, we can apply it directly. If $\hat{m}=1$ we observe that (11) holds by definition, since $M_{j} \geq 1=\hat{m}$. If $\hat{m}=1=M_{1}$ we have also to show that (12) is satisfied. This is true: indeed, by contradiction, if (12) were false then $\Omega_{1}=\sum_{i=1}^{n} a_{1 i} \omega_{i}$ violating the first order Melnikov condition (namely (4) with $|h| \leq 1)$. If $m=1$ then $\hat{m}=0,1$ and we are in one of the previous cases. We finally consider the case $\hat{m} \geq 2$. We have to prove (11). Note first that it is verified for $m=2$. Indeed in this case $\hat{m}=2$ and, hence, $\hat{\omega}=\omega$. By definition $M_{j} \Omega_{j}=a_{j} \cdot \omega$ for $j=1,2$. Again by the first order Melnikov condition we have, for $j=1,2$, that $M_{j} \geq 2$ which implies $M_{j} \geq 2=\hat{m}$ and (11) holds. All the other cases are covered by the hypothesis (iii). We can apply Lemma 2.3.

Let $t_{0} \leq \tau \leq t_{0}+T_{e}$ be the time found in Lemma 2.3 and consider the interval $\mathcal{J}:=2 \pi \tau+2 \pi[-\Theta, \Theta] \subset\left[t_{0}-2 \pi \Theta, t_{0}+2 \pi T_{e}+2 \pi \Theta\right] . \quad \forall T=$ $2 \pi \tau+2 \pi \theta \in \mathcal{J}$ (i.e. $|\theta| \leq \Theta$ ) formula (72) becomes

$$
\Omega_{\eta j} T=2 \pi\left(\Omega_{j}(\tau+\theta)-\left(\mathcal{Q R}^{-1}\right)_{j}\langle\omega(\tau+\theta)\rangle\right) \quad \forall 1 \leq j \leq m
$$

By (13), $\forall 1 \leq i \leq n$ there exists a $k_{i} \in \mathbb{Z}$ such that $\omega_{i} \tau=k_{i}+\left\langle\omega_{i} \tau\right\rangle$ with $\left|\left\langle\omega_{i} \tau\right\rangle\right| \leq \delta$; moreover $\left|\omega_{i} \theta\right| \leq \delta / 4$ by definition of $\Theta$. Hence we have $\left|\omega_{i}(\tau+\theta)-k_{i}\right| \leq \delta+\delta / 4<1 / 2$ being $\delta \leq 1 / 4$. So $\left\langle\omega_{i}(\tau+\theta)\right\rangle=\omega_{i}(\tau+\theta)-k_{i}$ and

$$
\left|\left\langle\omega_{i}(\tau+\theta)\right\rangle\right| \leq \frac{5}{4} \delta, \quad \forall 1 \leq i \leq n
$$

For $1 \leq j \leq m$, by (14) we have $\operatorname{dist}\left(\Omega_{j} \tau, \mathbb{Z}\right) \geq d_{0}$, hence, by the definition of $\Theta, \operatorname{dist}\left(\Omega_{j}(\tau+\theta), \mathbb{Z}\right) \geq d_{0}-d_{0} / 8$. Collecting the previous inequalities and (79), from (78) we obtain

$$
\begin{aligned}
\operatorname{dist}\left(\Omega_{\eta j} T, 2 \pi \mathbb{Z}\right) & =2 \pi \operatorname{dist}\left(\Omega_{j}(\tau+\theta)-\left(\mathcal{Q R} \mathcal{R}^{-1}\right)_{j}\langle\omega(\tau+\theta)\rangle, \mathbb{Z}\right) \\
& \geq 2 \pi\left(d_{0}-\frac{d_{0}}{8}-\frac{5 d_{0}}{8}\right)=\frac{\pi d_{0}}{2}
\end{aligned}
$$

by definition of $\delta$. Finally, we recall (73) to end the proof. 
Lemma 4.2. Let condition (b) of Theorem 1.1 hold, namely $\alpha>0$. Let

$$
\theta:=\min _{1 \leq i \leq n} \frac{1}{\left|\omega_{i}\right|} \quad \text { and } \quad d_{1}:=\min \left\{\frac{\pi}{8 m}, \frac{\pi \alpha \theta}{2 n m}\right\} .
$$

Then $\forall t_{0} \geq 0$ there exists an open set $\mathcal{A} \subset\left[t_{0}, t_{0}+4 \pi \theta\right]$ of measure, at least, $\pi \theta / n$, such that $\forall T \in \mathcal{A}, \mathcal{M}^{-1}:=\mathcal{M}^{-1}\left(I_{0}(T)\right.$, $\left.T\right)$ satisfies

$$
\left|\mathcal{M}^{-1}\right| \leq \frac{2}{d_{1}} .
$$

Proof. The function $T \rightarrow \Omega_{\eta}\left(I_{0}(T)\right) T$ is a piecewise smooth function with discontinuities at the points $T_{i, k}=\frac{2 \pi}{\omega_{i}}\left(k+\frac{1}{2}\right), 1 \leq i \leq n, k \in \mathbb{Z}$. Apart these points $\Omega_{\eta}\left(I_{0}(T)\right) T$ is differentiable w.r.t. to $T$ and has constant derivative $\Omega-\mathcal{Q R}^{-1} \omega=: \xi \in \mathbb{R}^{m}$. By the definition of $\theta$, in every interval of the type $(2 \pi \theta(h-1 / 2), 2 \pi \theta(h+1 / 2)), h \in \mathbb{Z}$, fall at most $n-1$ points of discontinuity and, hence, there exists an interval $\mathcal{J}_{1} \subseteq(2 \pi \theta(h-1 / 2), 2 \pi \theta(h+1 / 2))$ of length, at least, $\Delta:=2 \pi \theta / n$, in which $\Omega_{\eta}\left(I_{0}(T)\right) T$ is smooth. Hence, on $\mathcal{J}_{1}$, by (72), $\Omega_{\eta} T=x+\xi T$ for a suitable $x \in \mathbb{R}^{m}$.

For $1 \leq j \leq m$, let define the sets

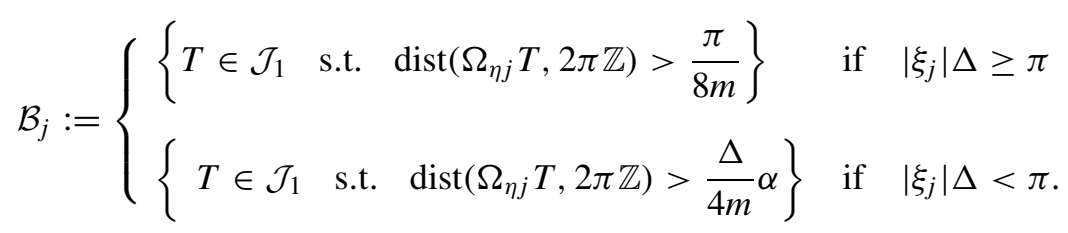

Remembering the definition $\alpha:=\min _{1 \leq j \leq m}\left|\xi_{j}\right|$, we note that meas $\left(\mathcal{B}_{j}\right) \geq \Delta(1-$ $\left.\frac{1}{2 m}\right), \forall 1 \leq j \leq m$. Let $\mathcal{A}:=\cap_{j=1}^{m} \mathcal{B}_{j}$, then $\mathcal{A} \subseteq \mathcal{J}_{1}$ and meas $\left(\mathcal{J}_{1}\right) \geq \Delta / 2$. By construction $\forall T \in \mathcal{A}$, $\operatorname{dist}\left(\Omega_{\eta j} T, 2 \pi \mathbb{Z}\right) \geq d_{1}, \forall 1 \leq j \leq m$. Finally the Lemma follows from (73).

REMARK 4.1. Our non resonance conditions (a)-(b) of Theorem 1.1 are sharp: if (a)-(b) are violated, it is not possible in general to find a period $T$ is such a way that the matrix $\mathcal{M}$ defined in (69)-(72) is invertible (clearly it must be $m \geq 3$ and $\hat{m} \geq 1$ ). As an example, consider the Hamiltonian $\mathcal{H}_{*}=$

$$
\begin{aligned}
\omega \cdot \mathcal{I}_{*} & +\Omega Z_{*} \cdot \bar{Z}_{*}+\frac{1}{2}\left|\mathcal{I}_{*}\right|^{2}+\frac{1}{p} \sum_{j=1}^{\hat{m}}\left(a_{j 1} \mathcal{I}_{* 1}\right. \\
& \left.+a_{j 2} \mathcal{I}_{* 2}\right) Z_{* j} \bar{Z}_{* j}+\sum_{2|k|+|a+\bar{a}| \geq 6} R_{k, a, \bar{a}}^{*}\left(\varphi_{*}\right) \mathcal{I}_{*}^{k} Z_{*}^{a} \bar{Z}_{*}^{\bar{a}},
\end{aligned}
$$

with $m \geq 4(\hat{m} \leq m)$ and

$$
a_{j 1}:=\left\{\begin{array}{ll}
1 & \text { if } \quad 1 \leq j \leq \hat{m}-1 \\
0 & \text { if } \quad j=\hat{m}
\end{array} \quad a_{j 2}:=\left\{\begin{array}{lll}
j & \text { if } \quad 1 \leq j \leq \hat{m}-1 \\
1 & \text { if } \quad j=\hat{m}
\end{array}\right.\right.
$$


The Hamiltonian $\mathcal{H}_{*}$ is in the form (1)-(5). We choose $\hat{m}$ so that $p:=\hat{m}-1$ is a prime integer with $3 \leq p \leq m-1$ (we require $p \neq 1,2$ since $(\omega, \Omega)$ must satisfy the second order Melnikov non-resonance conditions (4) and $(\omega, \Omega)$ are related by (81) below). Let also $\hat{\omega}=\left(\omega, \Omega_{\hat{m}+1}, \ldots, \Omega_{m}\right)$ be any rationally independent vector in $\mathbb{R}^{\hat{n}}$ and define

$$
\Omega_{j}:=\frac{1}{p}\left(a_{j 1} \omega_{1}+a_{j 2} \omega_{2}\right), \quad \forall 1 \leq j \leq \hat{m} .
$$

Note that, by (81), the equations (5) are fulfilled with $M_{j}=p, \forall 1 \leq j \leq \hat{m}$. Hence $M_{j}=p=\hat{m}-1<\hat{m}$ and condition (a)-(iii) is violated. After the rescaling in (27), the new Hamiltonian is $\mathcal{H}=\omega \cdot \mathcal{I}+\Omega Z \cdot \bar{Z}+\eta^{2}\left(\frac{1}{2}|\mathcal{I}|^{2}+\mathcal{Q} I\right.$. $Z \bar{Z})+O\left(\eta^{4}\right)$ where $\mathcal{Q} \in \operatorname{Mat}(m \times n, \mathbb{R})$ is defined by

$$
\mathcal{Q}_{j i}= \begin{cases}a_{j i} / p & \text { if } 1 \leq j \leq \hat{m}, \quad i=1,2, \\ 0 & \text { elsewhere }\end{cases}
$$

Since the Hamiltonian $\mathcal{H}$ does not contain terms of the form (29)-(30)-(31)(32), $\mathcal{H}$ is yet in the normal form (33) (i.e. (60)) with $\mathcal{R}=\mathbf{1}_{n}$. Therefore $\left(\Omega-\mathcal{Q R}^{-1} \omega\right)_{j}=(\Omega-\mathcal{Q} \omega)_{j}=0, \forall 1 \leq j \leq \hat{m}$, by (81), and also condition (b) is violated. Now, whatever we choose $T \in \mathbb{R}, I_{0} \in \mathbb{R}^{n}, n \in \mathbb{Z}^{n}$ such that $\omega+\eta^{2} \mathcal{R} I_{0}=2 \pi n / T$, i.e. (67) is satisfied, we get, substituting $I_{0}=$ $\eta^{-2} \mathcal{R}^{-1}((2 \pi n / T)-\omega)$ into $(66), \Omega_{\eta}\left(I_{0}\right) T=(\Omega-\mathcal{Q} \omega) T+2 \pi \mathcal{Q} n$. Hence, $\forall 1 \leq j \leq m$

$$
\Omega_{\eta j} T=\Omega_{\eta j}\left(I_{0}\right) T=2 \pi\left(\mathcal{Q}_{j 1} n_{1}+\mathcal{Q}_{j 2} n_{2}\right) .
$$

We claim that, for all $n_{i} \in \mathbb{Z}$, at least one $\Omega_{\eta j} T$ is an integer multiple of $2 \pi$ and hence the matrix $\mathcal{M}:=\mathbf{1}_{m}-e^{\mathrm{i} \Omega_{\eta} T}$ has a zero eigenvalue. In fact,

$$
\mathcal{Q}_{j 1} n_{1}+\mathcal{Q}_{j 2} n_{2}= \begin{cases}\left(n_{1}+n_{2} j\right) / p & \text { if } \quad 1 \leq j \leq p \\ n_{2} / p & \text { if } j=\hat{m}\end{cases}
$$

Thus, if $n_{2}$ is a multiple of $p$, then $\Omega_{\eta \hat{m}} T \in 2 \pi \mathbb{Z}$. Otherwise, let $j^{*}$ be the unique solution of the linear congruence $n_{2} j^{*} \equiv-n_{1} \bmod p$ (recall that $p$ is prime). In this case $\Omega_{\eta j *} T \in 2 \pi \mathbb{Z}$. In both cases $\mathcal{M}$ is not invertible.

In the next lemma we prove the existence of suitable pseudo $T$-periodic solutions of the Hamiltonian system (62) close to the manifold $\mathcal{P}$. Roughly, by the "twist condition" $\operatorname{det} \mathcal{R} \neq 0$ and the nonresonance property (69), the manifold $\mathcal{P}$ is "non-degenerate", i.e. the only $T$-periodic solutions of $H_{\text {int }}$, close to $\mathcal{P}$, are the set $\mathcal{P}$. This implies, by the Contraction Mapping Theorem, the existence of a manifold of pseudo $T$-periodic solutions $\zeta_{\phi_{0}}$, close to $\mathcal{P}$, diffeomorphic to $\mathbb{T}^{n}$. $\zeta_{\phi_{0}}$ are solutions of (61) for all $t \in(0, T)$ and satisfy $\phi(T)=\phi(0)=\phi_{0}, z(T)=z(0)$ but it may happen that $I(T) \neq I(0)$. 
Lemma 4.3. Assume that Condition (a) or (b) of Theorem 1.1 holds. Then, there exist $\eta_{0}, C_{0}, C>0$, such that: $\forall \eta \in\left(0, \eta_{0}\right]$, there exist an open set $\mathcal{A}_{\eta} \subseteq$ $\left[\frac{1}{\eta^{2}}, \frac{1}{\eta^{2}}+C_{0}\right]$ of measure greater than $1 / C_{0}$ such that $\forall T \in \mathcal{A}_{\eta}$ and $\forall \phi_{0} \in \mathbb{T}^{n}$ there exists a unique function

$\zeta_{\phi_{0}}:=\left(I_{\phi_{0}}, \phi_{\phi_{0}}, z_{\phi_{0}}\right) \in C\left([0, T], \mathbb{R}^{n} \times \mathbb{T}^{n} \times \mathbb{C}^{m}\right) \cap C^{1}\left((0, T), \mathbb{R}^{n} \times \mathbb{T}^{n} \times \mathbb{C}^{m}\right)$,

smooth in $\phi_{0}$, such that

(i) $\zeta_{\phi_{0}}(t)$ solves $(61)$ for all $t \in(0, T)$;

(ii) $\zeta_{\phi_{0}}$ satisfies $\phi_{\phi_{0}}(0)=\phi_{\phi_{0}}(T)=\phi_{0}, z_{\phi_{0}}(0)=z_{\phi_{0}}(T)$;

(iii) $\sup _{t \in[0, T]}\left(\left|I_{\phi_{0}}(t)-I_{0}\right|+\left|\phi_{\phi_{0}}(t)-\phi_{0}-\widetilde{\omega} t\right|+\left|z_{\phi_{0}}(t)\right|\right) \leq C \eta^{2}$.

where $I_{0}:=I_{0}(T)$ is defined in (70) and $\widetilde{\omega}:=\widetilde{\omega}(T):=\omega+\eta^{2} \mathcal{R} I_{0}$.

Proof. In order to define the set $\mathcal{A}_{\eta} \subseteq\left[\frac{1}{\eta^{2}}, \frac{1}{\eta^{2}}+C_{0}\right]$ of "non-resonant" periods $T$, we use Lemmata 4.1 or 4.2 (according whether condition (a) or (b) of Theorem 1.1 holds) with $t_{0}:=\eta^{-2} . \forall T \in \mathcal{A}_{\eta}$ we look for a solution $\zeta_{\phi_{0}}$ of (62) (i.e. (61)) of the form

$$
I_{\phi_{0}}(t)=I_{0}+\eta J(t), \quad \phi_{\phi_{0}}(t)=\phi_{0}+\widetilde{\omega} t+\eta \psi(t), \quad z_{\phi_{0}}(t)=\eta w(t),
$$

for suitable functions $(J, \psi, w):[0, T] \rightarrow \mathbb{R}^{n} \times \mathbb{T}^{n} \times \mathbb{C}^{m}$ satisfying $\psi(0)=$ $\psi(T)=0$ and $w(0)=w(T)$. The condition $\psi(0)=0$ is a "transversality condition": we impose the "correction" $(J, \psi, w)$ to belong to a supplementary linear space to the tangent space of the unperturbed manifold $\mathcal{P}$ (see [BB] for a discussion of the different supplementary spaces). The functions $(J, \psi, w)$ must satisfy the system

$$
\begin{aligned}
\dot{J} & =O\left(\eta^{3}\right), \\
\dot{\psi}-\eta^{2} J & =O\left(\eta^{3}\right) \\
\dot{w}_{j}-\mathrm{i} \Omega_{\eta j} w_{j} & =\mathrm{i} \eta^{2} \sum_{|a+\bar{a}|=3} R_{0, a, \bar{a}}^{*}\left(\phi_{0}+\widetilde{\omega} t\right) \bar{a}_{j} w^{a} \bar{w}^{\bar{a}-e_{j}}+O\left(\eta^{3}\right), j=1, \ldots, m
\end{aligned}
$$

where $\Omega_{\eta}=\Omega_{\eta}\left(I_{0}(T)\right) \in \operatorname{Mat}(m \times m, \mathbb{R})$ is the real diagonal matrix defined in (66).

In order to find $(J, \psi, w)$ we use Lemmata 2.4 and 2.5. In connection with the notation of Lemma 2.5 we have here $M=\eta^{2} \mathcal{R}$ and $\mathcal{M}=\mathbf{1}_{m}-e^{\mathrm{i} \Omega_{\eta} T}$. Let $L$ denote the corresponding Green operator in (24) and $P:=P\left(J, \psi, w ; \phi_{0}\right)$ the right hand side of (83), (84), (85). It is sufficient to find a fixed point $(J, \psi, w) \in X$, space defined in (23), of

$$
(J, \psi, w)=L\left(P\left(J, \psi, w ; \phi_{0}\right)\right) .
$$

If $T$ is obtained in Lemma 4.1, resp. Lemma 4.2, we have, by (80), resp. (77), that $\left|\mathcal{M}^{-1}\right| \leq 2 / d_{1}$, resp. $\left|\mathcal{M}^{-1}\right| \leq 4 / \pi d_{0}$. Moreover $|M|=O\left(\eta^{2}\right),\left|e^{\mathrm{i} \Omega_{\eta}}\right|=$ 
$\left|\operatorname{diag}\left\{e^{\mathrm{i} \Omega_{\eta j}}\right\}\right| \leq m, T=O\left(\eta^{-2}\right)$ and, hence, from (25), we get the estimate on the Green operator $|L| \leq C^{\prime} \eta^{-2}$ for some $C^{\prime}>0$. Moreover $P\left(0 ; \phi_{0}\right)=O\left(\eta^{3}\right)$. Then, for a constant $C$ large enough we have $\delta_{0}:=C \eta \geq 2|L(P(0))|$. Finally, since $\sup _{B_{\delta_{0}}}|D P|=O\left(\eta^{3}\right)$, for $\eta$ small enough, we verify also (21). Applying Lemma 2.4 in the ball $B_{\delta_{0}}$ we prove the existence of a solution $\zeta_{\phi_{0}}$ satisfying $\left\|\zeta_{\phi_{0}}\right\| \leq C \eta$, and hence, by (82), we get the estimate (iii).

Since $\zeta_{\phi_{0}} \in X$ solves the integral system (86), $\zeta_{\phi_{0}}(t)$ is actually $C^{1}$ for all $t \in(0, T)$ and solves (61).

Finally, since the operator $\phi_{0} \rightarrow L P\left(J, \psi, w ; \phi_{0}\right)$ is smooth, by the Implicit Function Theorem, we also deduce that the function $\phi_{0} \rightarrow \zeta_{\phi_{0}}$ is $C^{1}$.

REMARK 4.2. The terms (29), (30), (31), (32), that have been "averaged out" in Proposition 3.1, could not have been treated perturbatively in the previous Lemma. Indeed (29) (resp. (32)) would have given rise to a term $O(1)$ (resp. $O\left(\eta^{2}\right)$ ) in (85); (30) to a term $O(\eta)$ in (83); and (31) to a term $O\left(\eta^{2}\right)$ in (85). Roughly speaking, since the period $T \geq 1 / \eta^{2}$, only terms of magnitude $o\left(\eta^{2}\right)$ can be dealt as perturbations.

REMARK 4.3. The invertibility of the twist matrix $\mathcal{R}$ has been used in two ways: to modulate the frequency $\omega$ yielding the existence of nearby resonant frequencies, see (67), and to imply that the manifold $\mathcal{P}$ is non-degenerate (together with the non-resonance property (69)). Weaker conditions could in principle be used (if the elliptic variables were absent think to [CZ1]). However, in this case, when $\mathcal{R}$ is degenerate one is forced to study the higher order terms in the normal form of Proposition 3.1 making the perturbative analysis much more difficult. Moreover in the three body problem application we will prove that $\mathcal{R}$ is invertible, see Lemma 5.1.

REMARK 4.4. When $m=0$, namely in the case of maximal tori, the twist matrix $\mathcal{R}$ reduces simply to the matrix of the second derivatives of $\mathcal{H}_{*}$ w.r.t. the action variables $\mathcal{I}_{*}$. This is not the case when $m \neq 0$. Consider the Hamiltonian $\mathcal{H}_{*}=\omega \mathcal{I}_{*}+\Omega Z_{*} \bar{Z}_{*}+\mathcal{I}_{*}\left(Z_{*}+\bar{Z}_{*}\right)+\mathcal{I}_{*}^{2} / \Omega$ where $\left(\mathcal{I}_{*}, \varphi_{*}\right) \in \mathbb{R} \times \mathbb{T}$, $\left(Z_{*}, \bar{Z}_{*}\right) \in \mathbb{C}^{2}$ and $\Omega>0$. The twist matrix in (7) turns out to be $\mathcal{R}=0$ although the second derivative of $\mathcal{H}_{*}$ w.r.t. $\mathcal{I}_{*}$ equals to $2 / \Omega \neq 0$. Heuristically, for lower dimensional elliptic tori, the twist matrix $\mathcal{R}$ takes into account, with the second addendum in the r.h.s. of (7), the interaction between the torus variables $\left(\mathcal{I}_{*}, \varphi_{*}\right)$ and the elliptic variables $Z_{*}$. Finally observe that the symplectic map $\mathcal{I}_{*}=I, \varphi_{*}=\phi+(z-\bar{z}) /(\mathrm{i} \Omega), Z_{*}=z-I / \Omega$ (which can be found through the averaging procedure of Section 3 ) transforms $\mathcal{H}$ into the isochronous Hamiltonian $H=\omega I+\Omega z \bar{z}$.

\section{2. - The variational principle}

We now define the "reduced Hamiltonian action functional" $\mathcal{E}: \mathbb{T}^{n} \rightarrow \mathbb{R}$ as the Hamiltonian action functional $\mathcal{A}$ evaluated on the pseudo $T$-periodic 
solutions $\zeta_{\phi_{0}}$ obtained in Lemma 4.3, namely ${ }^{(18)}$

$$
\begin{aligned}
\mathcal{E}\left(\phi_{0}\right): & =\mathcal{A}\left(I_{\phi_{0}}, \phi_{\phi_{0}}, z_{\phi_{0}}, \bar{z}_{\phi_{0}}\right) \\
& =\int_{0}^{T} I_{\phi_{0}} \cdot \dot{\phi}_{\phi_{0}}+\mathrm{i} z_{\phi_{0}} \cdot \dot{\bar{z}}_{\phi_{0}}-H\left(I_{\phi_{0}}, \phi_{\phi_{0}}, z_{\phi_{0}}, \bar{z}_{\phi_{0}}\right) d t .
\end{aligned}
$$

By Lemma 4.3 the reduced action functional $\mathcal{E}$ is smooth in $\phi_{0}$.

Critical points of $\mathcal{E}: \mathbb{T}^{n} \rightarrow \mathbb{R}$ give rise to $T$-periodic solutions of the Hamiltonian system (61), according to the following Lemma:

LEMMA 4.4. $\partial_{\phi_{0}} \mathcal{E}\left(\phi_{0}\right)=I_{\phi_{0}}(T)-I_{\phi_{0}}(0)$. Hence, if $\phi^{\star} \in \mathbb{T}^{n}$ is a critical point of $\mathcal{E}$, then $\zeta_{\phi^{\star}}(t)$ can be extended to a $T$-periodic solution of the Hamiltonian system (61).

ProOF. Differentiating w.r.t. $\phi_{0}$ in (87) we get

$$
\begin{aligned}
\partial_{\phi_{0}} \mathcal{E}\left(\phi_{0}\right)= & \int_{0}^{T} \partial_{\phi_{0}} I_{\phi_{0}} \cdot \dot{\phi}_{\phi_{0}}+I_{\phi_{0}} \cdot \partial_{\phi_{0}} \dot{\phi}_{\phi_{0}}+\mathrm{i} \partial_{\phi_{0}} z_{\phi_{0}} \dot{\bar{z}}_{\phi_{0}}+\mathrm{i} z_{\phi_{0}} \partial_{\phi_{0}} \dot{\bar{z}}_{\phi_{0}} \\
& -\partial_{I} H \partial_{\phi_{0}} I_{\phi_{0}}-\partial_{\phi} H \partial_{\phi_{0}} \phi_{\phi_{0}}-\partial_{z} H \partial_{\phi_{0}} z_{\phi_{0}}-\partial_{\bar{z}} H \partial_{\phi_{0}} \bar{z}_{\phi_{0}} d t \\
= & I_{\phi_{0}}(T) \partial_{\phi_{0}} \phi_{\phi_{0}}(T)-I_{\phi_{0}}(0) \partial_{\phi_{0}} \phi_{\phi_{0}}(0)+\mathrm{i} \partial_{\phi_{0}} \bar{z}_{\phi_{0}}(T) z_{\phi_{0}}(T) \\
& -\mathrm{i} \partial_{\phi_{0}} \bar{z}_{\phi_{0}}(0) z_{\phi_{0}}(0),
\end{aligned}
$$

by an integration by parts, and since $\zeta_{\phi_{0}}(t)$ satisfies the Hamilton's equations (61) in $(0, T)$. Moreover, since $\forall \phi_{0} \in \mathbb{T}^{n}, \phi_{\phi_{0}}(T)=\phi_{\phi_{0}}(0)=\phi_{0}$ and $\bar{z}_{\phi_{0}}(T)=$ $\bar{z}_{\phi_{0}}(0)$, deriving w.r.t. $\phi_{0}$, we get $\partial_{\phi_{0}} \phi_{\phi_{0}}(T)=\partial_{\phi_{0}} \phi_{\phi_{0}}(0)=\mathbf{1}_{n}$ and $\partial_{\phi_{0}} \bar{z}_{\phi_{0}}(T)=$ $\partial_{\phi_{0}} \bar{z}_{\phi_{0}}(0)$. This implies $\partial_{\phi_{0}} \mathcal{E}\left(\phi_{0}\right)=I_{\phi_{0}}(T)-I_{\phi_{0}}(0)$. Hence, if $\phi^{\star} \in \mathbb{T}^{n}$ is a critical point of $\mathcal{E}, \zeta_{\phi^{\star}}(T)=\zeta_{\phi^{\star}}(0)$ and we deduce that $\zeta_{\phi^{\star}}(t)$ can be extended to a $T$-periodic solution of the Hamiltonian system (61).

The following Lemma, which is a consequence of the autonomy of the Hamiltonian $H$, holds.

Lemma 4.5. $\forall \phi_{0} \in \mathbb{T}^{n}$ there exists $V\left(\phi_{0}, \eta\right) \in \mathbb{R}^{n}$ with $V\left(\phi_{0}, \eta\right)=\widetilde{\omega}+O\left(\eta^{3}\right)$ such that

$$
V\left(\phi_{0}, \eta\right) \cdot \partial_{\phi_{0}} \mathcal{E}\left(\phi_{0}\right)=0, \quad \forall \phi_{0} \in \mathbb{T}^{n} .
$$

Proof. Since $\zeta_{\phi_{0}}=\left(I_{\phi_{0}}, \phi_{\phi_{0}}, z_{\phi_{0}}\right)$ satisfies the Hamiltonian system (61) in $(0, T)$ and $\phi_{\phi_{0}}(T)=\phi_{\phi_{0}}(0)=\phi_{0}, z_{\phi_{0}}(T)=z_{\phi_{0}}(0)$, then

$$
H\left(I_{\phi_{0}}(T), \phi_{0}, z_{\phi_{0}}(0), \bar{z}_{\phi_{0}}(0)\right)=H\left(I_{\phi_{0}}(0), \phi_{0}, z_{\phi_{0}}(0), \bar{z}_{\phi_{0}}(0)\right) .
$$

By the mean value theorem there exists $\xi_{\phi_{0}}$ in the segment between $\left[I_{\phi_{0}}(T), I_{\phi_{0}}(0)\right]$ such that

$$
\partial_{I} H\left(\xi_{\phi_{0}}, \phi_{0}, z_{\phi_{0}}(0), \bar{z}_{\phi_{0}}(0)\right) \cdot\left[I_{\phi_{0}}(T)-I_{\phi_{0}}(0)\right]=0 .
$$

${ }^{(18)} \mathcal{E}\left(\phi_{0}\right) \in \mathbb{R}$ since $\int_{0}^{T} \operatorname{Im}\left(\mathrm{i} z_{\phi_{0}} \dot{\bar{z}}_{\phi_{0}}\right) d t=\int_{0}^{T} \operatorname{Re}\left(z_{\phi_{0}} \dot{\bar{z}}_{\phi_{0}}\right) d t=\int_{0}^{T} \frac{1}{2} \frac{d}{d t}\left(z_{\phi_{0}} \bar{z}_{\phi_{0}}\right) d t=0$. 
Define $V\left(\phi_{0}, \eta\right):=\partial_{I} H\left(\xi_{\phi_{0}}, \phi_{0}, z_{\phi_{0}}(0), \bar{z}_{\phi_{0}}(0)\right) . \quad$ By Lemma 4.4 and (90), formula (88) follows. Finally, since $I_{\phi_{0}}(T)-I_{0}=I_{\phi_{0}}(0)-I_{0}=O\left(\eta^{2}\right)$, $z_{\phi_{0}}(0)=O\left(\eta^{2}\right)$ and $\partial_{I} H=\omega+\eta^{2} \mathcal{R} I+\eta^{2} \mathcal{Q}^{T} z \bar{z}+O\left(\eta^{3}\right)$, we deduce the estimate $V\left(\phi_{0}, \eta\right)=\widetilde{\omega}+O\left(\eta^{3}\right)$.

Proof of Theorem 1.1. By Lemma 4.4 the absolute minimum (and maximum) $\phi^{\star} \in \mathbb{T}^{n}$ gives rise to a $T$-periodic solution $\zeta_{\phi^{\star}}$ of (61). However one expects the existence of at least $n$ geometrically distinct $T$-periodic solutions of (61), i.e. solutions not obtained one from each other simply by time-translations.

In order find multiple geometrically distinct periodic solutions of (61) we restrict the reduced action function $\mathcal{E}$ to the plane $E:=[\widetilde{\omega}]^{\perp}$ orthogonal to the periodic flow $\widetilde{\omega}=(1 / T) 2 \pi k$ with $k \in \mathbb{Z}^{n}$. The set $\mathbb{Z}^{n} \cap E$ is a lattice of $E$, (see for example Lemma 8.2 of [BBB]) and hence $\mathcal{E}$ can be defined on the quotient space $\Gamma:=E /\left(\mathbb{Z}^{n} \cap E\right) \sim \mathbb{T}^{n-1}$.

A critical point $\phi^{\star}$ of $\mathcal{E}: \Gamma \rightarrow \mathbb{R}$ is a critical point of $\mathcal{E}: \mathbb{T}^{n} \rightarrow \mathbb{R}$. Indeed, since the tangent space $T_{\phi_{0}} \Gamma=[\widetilde{\omega}]^{\perp}$, then

$$
\partial_{\phi_{0}} \mathcal{E}\left(\phi^{\star}\right)=\lambda\left(\phi^{\star}\right) \widetilde{\omega}
$$

for some Lagrange multiplier $\lambda\left(\phi^{\star}\right) \in \mathbb{R}$. (91) and Lemma 4.5 imply that $\lambda\left(\phi^{\star}\right)\left(|\widetilde{\omega}|^{2}+O\left(\eta^{3}\right)\right)=0$ and so, for $\eta$ small, $\lambda\left(\phi^{\star}\right)=0$.

By the Lusternik-Schnirelman category theory, see for example $[\mathrm{Am}]$, since cat $\Gamma=\operatorname{cat} \mathbb{T}^{n-1}=n$, we can define the $n$ min-max critical values $c_{1} \leq c_{2} \leq \ldots \leq$ $c_{n}$ for the reduced action functional $\mathcal{E}_{\mid \Gamma}$. Let $\phi_{1}^{\star}, \ldots, \phi_{n}^{\star} \in \Gamma$ be $n$ corresponding critical points. If the critical levels $c_{i}$ are all distinct, the $T$-periodic solutions $\zeta_{\phi_{i}^{\star}}(t)=\left(I_{0}, \phi_{i}^{\star}+\widetilde{\omega} t, 0\right)+O\left(\eta^{2}\right)$ of $(61)$ are geometrically distinct, since their actions $\mathcal{A}\left(\zeta_{\phi_{i}^{\star}}\right)=\mathcal{E}\left(\phi_{i}^{\star}\right)=c_{i}$ are different. On the other hand, if some minmax critical level $c_{i}$ coincide, then $\mathcal{E}_{\mid \Gamma}$ possesses infinitely many critical points. However not all the corresponding $T$-periodic solutions of (61) are necessarily geometrically distinct, since two different critical points could belong to the same orbit. In any case, since one periodic solution can cross $\Gamma$ at most a finite number of times, the existence of infinitely many geometrically distinct orbits of (61) follows ${ }^{(19)}$.

Finally, under the inverse transformation of $\Phi$, defined in Proposition 3.1, $\eta$-close to the identity and the inverse transformation of (27), we find the $T$ periodic solutions $\zeta_{\eta}$ of Theorem 1.1 satisfying the estimates (i)-(ii).

The statement on the minimal period descends from the following Proposition.

PROPOSITION 4.1. Let $\zeta(t)=(I(t), \phi(t), z(t))$ be a $T$-periodic solution of $(62)$ with $\mathrm{C}^{-2} \geq T \geq T_{0}$ for some $T_{0}$ large, independent of $\eta$. Then the minimal period $T_{\min }$ of $\zeta$ satisfies $T_{\min } \geq$ const $T^{1 /(\tau+1)}$.

${ }^{(19)}$ Non-degenerate critical points of the Poincaré-Melnikov primitive $\phi_{0} \rightarrow \int_{0}^{T}\left(H-H_{\text {int }}\right)\left(I_{0}, \phi_{0}+\right.$ $\widetilde{\omega} t, 0) d t$ could be continued to solutions of (61). However, it is very difficult to compute this function, its critical points and check whether they are non-degenerate (if ever true). 
Proof. Since $\zeta$ is $T$-periodic, $\phi(T)-\phi(0)=2 \pi k$ for some $k \in \mathbb{Z}^{n}$. We claim that $T_{\min } \geq T / g$ where $g:=\operatorname{gcd}\left(k_{1}, \ldots k_{n}\right)$. Indeed $T_{\min } \geq T_{\min }^{\phi}$ where $T_{\min }^{\phi}$ is the minimal period of $\phi(t)$. Moreover $T_{\min }^{\phi}=T / n$ for some integer $n \geq 1$ and $\phi\left(T_{\min }\right)-\phi(0)=2 \pi \widetilde{k}$ for some $\widetilde{k} \in \mathbb{Z}^{n}$. It follows that $\widetilde{k} n=k$ and so $n \leq g$, proving the claim.

By the second equation in (62) we also have $\phi(T)-\phi(0)=\omega T+O\left(\eta^{2} T\right)$ and then, letting $\hat{k}:=k / g \in \mathbb{Z}^{n}, 2 \pi g \hat{k}=\omega T+O\left(\eta^{2} T\right)$. We deduce that

$$
T_{\min } \geq \frac{T}{g} \geq \frac{2 \pi}{|\omega|}|\hat{k}|-O\left(\frac{\eta^{2} T}{|\omega| g}\right) \geq \frac{2 \pi}{|\omega|}|\hat{k}|-O\left(\frac{C}{|\omega|}\right),
$$

since $\eta^{2} T \leq C$. Let now choose $h \in \mathbb{Z}^{n} \backslash\{0\}$ with $|h| \leq|\hat{k}|$ such that $\hat{k} \cdot h=0$. Multiplying by $h$ we get $0=2 \pi g \hat{k} \cdot h=\omega \cdot h T+O\left(\eta^{2} T|h|\right)$ and so $\omega \cdot h=O\left(\eta^{2}|h|\right)$. Using the Diophantine character of $\omega$ assumed in (4), namely $|\omega \cdot h| \geq \gamma(1+|h|)^{-\tau}, \forall h \in \mathbb{Z}^{n}$, we obtain $|h|^{\tau+1} \geq$ const $\eta^{-2} \geq$ const $T$. Hence $|\hat{k}|^{\tau+1} \geq|h|^{\tau+1} \geq$ const $T$ and $|\hat{k}| \geq$ const $T^{1 /(\tau+1)}$. The proposition follows from (92) for $T \geq T_{0}$ large enough.

Remark 4.5. The periodic orbits $\zeta_{\eta}$ found in Theorem 1.1 are $O\left(\eta^{2}\right)$ close to the torus $\mathcal{T}$ since $I_{0}$, defined in (70), satisfies $I_{0}=O(1)$. We could also try to find periodic solutions when e.g. $I_{0}=O(1 / \eta)$ and so $T=O(1 / \eta)$. However the terms $O\left(I^{k}\right)=O\left(\eta^{-k}\right)$ would be more difficult to control.

\section{5. - The planetary spatial three-body problem}

In this section we will prove the existence of periodic orbits of the planetary non planar three body problem, with "small eccentricities" and "small mutual inclinations", accumulating onto two-dimensional elliptic invariant tori. We first discuss the classical Hamiltonian formulation of this problem which dates back to Delaunay and Poincaré. For a detailed treatment see the Appendix of [BCV].

The three massive points ("bodies") $P_{0}, P_{1}, P_{2}$, with masses $m_{0}, m_{1}, m_{2}$, interact one each other through gravity (with constant of gravitation 1). Assume that the masses of the bodies satisfy, for some $0<\bar{\kappa} \leq 1$,

$$
\bar{\kappa} \varepsilon \leq \frac{m_{1}}{m_{0}}, \frac{m_{2}}{m_{0}} \leq \varepsilon \leq 1 .
$$

The number $\varepsilon>0$ is regarded as a small parameter: the point $P_{0}$ represents "the star" and the points $P_{1}$ and $P_{2}$ "the planets".

We now recall the classical definition of the "osculating ellipses" (at time $\left.t_{0}\right)$ of the two-body problems associated to the planets $P_{i}(i=1,2)$ and the 
star $P_{0}$. Let $u^{(0)}$ and $u^{(i)}$ denote the coordinates of the points $P_{0}$ and $P_{i}$ at time $t_{0}$ and let $\dot{u}^{(0)}$ and $\dot{u}^{(i)}$ denote the respective velocities. The "osculating plane" is defined as the plane spanned by $\left(u^{(i)}-u^{(0)}\right)$ and $\left(\dot{u}^{(i)}-\dot{u}^{(0)}\right)$; the "osculating ellipse" is defined as the Keplerian ellipse (lying on the osculating plane) defined by the Kepler solution, with initial data $\left(u^{(0)}, u^{(i)}\right)$ and $\left(\dot{u}^{(i)}-\dot{u}^{(0)}\right)$, of the two-body problem $\left(P_{0}, P_{i}\right)$ obtained disregarding (for $\left.t \geq t_{0}\right)$ the third body $P_{j}(j \neq i)$.

We assume that the eccentricities of such ellipses are small and that the intersection angle between the two planes containing the two osculating ellipses (usually referred as "mutual inclination") is also small. It is customary in celestial mechanics to denote the major semi-axes of such ellipses by $a_{i}$ and their eccentricities by $e_{i}$. Let

$$
\Lambda_{i}^{*}:=\kappa_{i}^{*} \sqrt{a_{i}}, \quad \kappa_{i}^{*}:=\frac{m_{i}}{\varepsilon} \frac{1}{\sqrt{m_{0}\left(m_{0}+m_{i}\right)}}
$$

( $\kappa_{i}^{*}$ is a dimensionless constant satisfying $\frac{\bar{\kappa}}{\sqrt{2}}<\kappa_{i}^{*}<1$ ). Since we are interested in small eccentricities, collisions are avoided by requiring that the major semiaxes $a_{i}=a_{i}\left(\Lambda^{*}\right):=\left(\Lambda_{i}^{*} / \kappa_{i}^{*}\right)^{2}, i=1,2$, are different, and different from zero. We, therefore, fix, once and for all,

$$
0<a_{\min }<a_{\max }
$$

and, from now on, we shall consider (attaching the index 1 to the "inner planet") values of $\Lambda^{*}$ in the set

$$
\mathcal{L}:=\left\{\Lambda^{*} \in \mathbb{R}^{2}: a_{\min } \leq a_{1}<a_{2} \leq a_{\max }\right\}
$$

The following classical result follows from the Delaunay-Poincare theory, see $[\mathrm{BCV}]$.

THEOREM (Delaunay-Poincaré). Fix $\Lambda_{0}^{*} \in \mathcal{L}$. There exists a symplectic set of variables $^{(20)}$

$$
\left(I_{0}, \varphi_{0}, p_{0}, q_{0}\right) \in \mathbb{R}^{2} \times \mathbb{T}^{2} \times \mathbb{R}^{2} \times \mathbb{R}^{2}
$$

where $I_{0}=\Lambda^{*} \in \mathcal{I}$ and $\mathcal{I} \subset \mathcal{L} \subset \mathbb{R}^{2}$ is a suitable two-dimensional open cube centered at $\Lambda_{0}^{*}$, such that the Hamiltonian of the spatial planetary three-body problem takes the form

$$
\mathcal{H}_{0}\left(I_{0}, \varphi_{0}, p_{0}, q_{0}\right)=h_{0}\left(I_{0}\right)+f_{0}\left(I_{0}, \varphi_{0}, p_{0}, q_{0}\right)
$$

${ }^{(20)}$ With symplectic form $d I_{0} \wedge d \varphi_{0}+d p_{0} \wedge d q_{0}$. 
with

$$
\begin{aligned}
& h_{0}:=-\frac{1}{2} \sum_{i=1}^{2} \frac{\kappa_{i}}{I_{0 i}^{2}}, \quad \kappa_{i}:=\left(\frac{m_{i}}{\varepsilon}\right)^{3} \frac{1}{m_{0}^{2}\left(m_{0}+m_{i}\right)}, \quad\left(\frac{\bar{\kappa}^{3}}{2}<\kappa_{i}<1\right), \\
& f_{0}:=\varepsilon f_{1}\left(I_{0}, p_{0}, q_{0}\right)+\varepsilon f_{2}\left(I_{0}, \varphi_{0}, p_{0}, q_{0}\right), \\
& f_{1}:=f_{1,0}\left(I_{0}\right)+\sum_{j=1}^{2} \bar{\Omega}_{j}\left(I_{0}\right)\left(p_{0 j}^{2}+q_{0 j}^{2}\right)+\widetilde{f}_{1}\left(I_{0}, p_{0}, q_{0}\right), \\
& \text { (97) } \int_{\mathbb{T}^{2}} f_{2} d \varphi_{0}=0, \quad \sup _{\substack{\mathcal{I}_{\sigma_{0}}^{2} \\
f_{1}}}
\end{aligned}
$$

$f_{1}$ and $f_{2}$ are real-analytic and uniformly bounded on ${ }^{(21)}$

$$
\mathcal{D}_{0}:=\mathcal{I}_{\sigma_{0}}^{2} \times \mathbb{T}_{s_{0}}^{2} \times D_{\rho_{0}}^{4} \subset \mathbb{C}^{8},
$$

where $\sigma_{0}, s_{0}, \rho_{0}$ are suitable positive numbers. Moreover $\widetilde{f}_{1}$ is even in $\left(p_{0}, q_{0}\right)$ and

$$
\begin{aligned}
& \inf _{I_{0} \in \mathcal{I}} \bar{\Omega}_{j}\left(I_{0}\right)>\inf _{I_{0} \in \mathcal{I}_{\sigma_{0}}^{2}}\left|\bar{\Omega}_{j}\left(I_{0}\right)\right|>\text { const }>0, \\
& \inf _{I_{0} \in \mathcal{I}}\left(\bar{\Omega}_{2}\left(I_{0}\right)-\bar{\Omega}_{1}\left(I_{0}\right)\right)>\inf _{I_{0} \in \mathcal{I}_{\sigma_{0}}^{2}}\left|\bar{\Omega}_{2}\left(I_{0}\right)-\bar{\Omega}_{1}\left(I_{0}\right)\right|>\text { const }>0 .
\end{aligned}
$$

We underline that the actions $I_{0}$ are simply $I_{0}=\Lambda^{*}$ and we refer to [BCV]-Theorem 1.1 for the complete expressions of the other Delaunay-Poincare variables $\left(\varphi_{0}, p_{0}, q_{0}\right)$.

We remark that the eccentricities $e_{i}$ are estimated as ${ }^{(22)}$

$$
c_{\mathcal{I}}^{-}\left|\left(p_{0}, q_{0}\right)\right| \leq e_{i} \leq c_{\mathcal{I}}^{+}\left|\left(p_{0}, q_{0}\right)\right|, \quad i=1,2
$$

for two suitable constants $0<c_{\mathcal{I}}^{-}<c_{\mathcal{I}}^{+}$(depending only on $\mathcal{I}$ ).

The Hamiltonian $\mathcal{H}_{0}$ in (96) describes a nearly-integrable, properly degenerate, system: the integrable Hamiltonian $h_{0}$ depends only on the two action variables $\left(I_{01}, I_{02}\right)$. Such degeneracies are a typical feature of problems arising in celestial mechanics and the source of the main difficulties (the application of standard KAM theory for finding maximal tori require the Hamiltonian to be non-degenerate). The frequency vector $\nabla h_{0}\left(I_{0}\right)$ is independent of $\varepsilon$, and then the conjugated angles $\varphi_{0}$ may be regarded as "fast angles" and, in "first approximation" the $\mathcal{H}_{0}$-motions are governed by the averaged Hamiltonian $h_{0}+\varepsilon f_{1}$. By (97), for any fixed $I_{0},\left\{\varphi_{0} \in \mathbb{T}^{2}\right\} \times\left\{p_{0}=q_{0}=0\right\}$ is an elliptic invariant torus for the averaged Hamiltonian $h_{0}+\varepsilon f_{1}$ run by the linear flow $\varphi_{0} \rightarrow \varphi_{0}+\nabla\left(h_{0}+\varepsilon f_{1,0}\right)\left(I_{0}\right) t$. These are the quasi-periodic motions that will persist, for $\varepsilon$ small, and $I_{0}$ in a nearly full (two-dimensional) measure set, as proved in $[\mathrm{BCV}]$, see also Theorem 5.2.

${ }^{(21)} D_{\rho}^{d} \subset \mathbb{C}^{d}$ denotes the open complex ball of radius $\rho$ centered at the origin.

${ }^{(22)}$ See (1.5),(C.10),(C.15),(C.17) of [BCV]. 
In the next proposition, through an appropriate averaging procedure, The Hamiltonian (96) is casted into a suitable normal form.

Proposition 5.1. Fix $N \in \mathbb{N}^{+}$. There exists a $O(\sqrt{\varepsilon})$-close to the identity, real analytic, symplectic change of variables $(J, \psi, z, \bar{z}) \in U_{\widetilde{r}}^{2} \times \mathbb{T}_{\widetilde{s}}^{2} \times D_{\widetilde{\rho}}^{4} \rightarrow$ $\left(I_{0}, \varphi_{0}, p_{0}, q_{0}\right) \in \mathcal{D}_{0} \subset \mathbb{C}^{8}$ transforming the Hamiltonian (96) into the real analytic Hamiltonian

$$
\mathcal{H}(J, \psi, z, \bar{z})=h_{\varepsilon}(J)+\varepsilon \widetilde{\Omega}(J) z \bar{z}+\varepsilon g(J, z, \bar{z} ; \varepsilon)+\varepsilon^{N} f(J, \psi, z, \bar{z} ; \varepsilon)
$$

where $h_{\varepsilon}:=h_{0}+O(\varepsilon)$,

$$
\begin{gathered}
\widetilde{r}=\text { const } \sqrt{\varepsilon}, \quad \widetilde{s}=\text { const }, \quad \widetilde{\rho}=\text { const }, \\
U:=\left\{J \in \mathcal{I}:\left|h_{0}^{\prime}(J) \cdot \ell\right| \geq \alpha_{0}, \forall \ell \in \mathbb{Z}^{2}, 0<|\ell| \leq K\right\} \subset \mathcal{I},
\end{gathered}
$$

is a closed set and

$$
K:=\frac{6(N-1)}{s_{0}} \log \frac{1}{\varepsilon}, \quad \alpha_{0}:=2 \sup _{\mathcal{I}_{0}}\left|h_{0}^{\prime \prime}\right| \widetilde{r} K=\text { const } \sqrt{\varepsilon} \log \frac{1}{\varepsilon},
$$

It results that

$$
\operatorname{meas}(\mathcal{I} \backslash U) \leq \text { const } \alpha_{0}=O\left(\sqrt{\varepsilon} \log \frac{1}{\varepsilon}\right) .
$$

Moreover

$$
\begin{gathered}
\sup _{J \in U_{\widetilde{r}}^{2}}|g(J, z, \bar{z})| \leq \text { const }(|z|+|\bar{z}|)^{3}, \\
\inf _{J \in U} \widetilde{\Omega}_{j}(J) \geq \inf _{J \in U_{\widetilde{r}}^{2}}\left|\widetilde{\Omega}_{j}(J)\right| \geq \chi_{0}>0, \\
\inf _{J \in U}\left(\widetilde{\Omega}_{2}(J)-\widetilde{\Omega}_{1}(J)\right) \geq \inf _{J \in U_{\widetilde{r}}^{2}}\left|\widetilde{\Omega}_{2}(J)-\widetilde{\Omega}_{1}(J)\right| \geq \chi_{0}>0,
\end{gathered}
$$

for some positive constant $\chi_{0}$.

PROOF. The Hamiltonian (101) has been deduced in [BCV] in the case $N=3$ and on the smaller domain $U^{\prime}:=\left\{J \in \mathcal{I}|| h_{0}^{\prime}(J) \cdot \ell \mid \geq \operatorname{const}\left(\sqrt{\varepsilon} \log ^{\tau+1}(1 / \varepsilon)\right) /(1+\right.$ $\left.\left.|l|^{\tau}\right), \forall \ell \in \mathbb{Z}^{2} \backslash\{0\}\right\} \subset U$, see formula (2.22) of [BCV] and introduce the complex coordinates (171). Note also that the analyticity constant $\widetilde{\rho}=$ const is bigger than $\widetilde{\rho}=\log ^{-1}(1 / \varepsilon)$ given in $[\mathrm{BCV}]$. For the proof of the proposition see the Appendix. 
The manifold $\{z=0\}$ is invariant under the Hamiltonian system generated by the integrable Hamiltonian $h_{\varepsilon}(J)+\varepsilon \widetilde{\Omega}(J) z \bar{z}$,

$$
\dot{J}=0, \quad \dot{\psi}=\partial_{J} h_{\varepsilon}(J)+\varepsilon \partial_{J} \widetilde{\Omega}(J) z \bar{z}, \quad \dot{z}=\mathrm{i} \varepsilon \widetilde{\Omega}(J) z
$$

and it is completely filled up by the invariant elliptic tori $\mathcal{T}\left(J_{0}\right):=\left\{J=J_{0}\right.$, $\left.\psi \in \mathbb{T}^{2}, z=0\right\}$, supporting the linear flow $t \rightarrow\left\{J_{0}, \psi_{0}+\omega_{\varepsilon}\left(J_{0}\right) t, 0\right\}$ with torus frequency $\omega_{\varepsilon}\left(J_{0}\right):=\partial_{J} h_{\varepsilon}\left(J_{0}\right)$. This family of 2-dimensional tori will not persist in its entirety for the complete Hamiltonian system generated by $\mathcal{H}$

$$
\dot{J}=-\partial_{\psi} \mathcal{H}, \quad \dot{\psi}=\partial_{J} \mathcal{H}, \quad \dot{z}=\mathrm{i} \partial_{\bar{z}} \mathcal{H}, \quad \dot{\bar{z}}=-\mathrm{i} \partial_{z} \mathcal{H}
$$

due to resonances among the oscillations. However, the persistence of a set of positive (2-dimensional) measure of perturbed elliptic invariant tori - those with Diophantine frequency $\omega_{\varepsilon}\left(J_{0}\right)$ - has been proved in [BCV], using the KAM theorem of [Pö2].

In Theorem 0.2 we will prove the existence of an abundance of periodic solutions with larger and larger period accumulating on each perturbed elliptic torus, applying Theorem 1.1. For this aim, we will first reprove (Theorem 5.2) the existence of the [BCV]-elliptic tori, using the KAM theorem of [Pö1] and furnishing also the KAM-normal form describing the dynamics in its neighborhood (it will be of the form considered in (1)). Moreover, for proving that the hypothesis of Theorem 1.1 are satisfied, we also need precise informations on the form of the KAM-transformation bringing into the KAM normal form, see Theorem 5.1-(ii).

For the existence result of the elliptic invariant tori (Theorem 5.2) and their surrounding periodic orbits (Theorem 0.2) it is sufficient, and we will assume, $N=3$.

First of all we rewrite the Hamiltonian $\mathcal{H}$ in (101) in a form suitable to apply the KAM theorem of [Pö1]. Introducing the coordinate $y \in \mathbb{R}^{n}$ around each torus $\mathcal{T}\left(J_{0}\right)$ in the usual way $J=J_{0}+y$, the Hamiltonian $\mathcal{H}$ can be developed as

$$
H(y, \psi, z, \bar{z} ; \omega):=\mathcal{H}\left(J_{0}+y, \psi, z, \bar{z}\right)=\mathcal{N}+P
$$

where $\mathcal{N}:=h_{\varepsilon}\left(J_{0}\right)+h_{\varepsilon}^{\prime}\left(J_{0}\right) \cdot y+\varepsilon \widetilde{\Omega}\left(J_{0}\right) z \bar{z}$ and $P=P\left(y, \psi, z, \bar{z} ; J_{0}\right)=P_{1}+$ $P_{2}+P_{3}+P_{4}$ with

$$
\begin{aligned}
P_{1} & :=h_{\varepsilon}\left(J_{0}+y\right)-h_{\varepsilon}\left(J_{0}\right)-h_{\varepsilon}^{\prime}\left(J_{0}\right) \cdot y=O\left(|y|^{2}\right), \\
P_{2} & :=\varepsilon\left(\widetilde{\Omega}\left(J_{0}+y\right)-\widetilde{\Omega}\left(J_{0}\right)\right) z \bar{z}=O(\varepsilon|y||z||\bar{z}|), \\
P_{3} & :=\varepsilon g\left(J_{0}+y, z, \bar{z}\right)=O\left(\varepsilon(|z|+|\bar{z}|)^{3}\right), \\
P_{4} & :=\varepsilon^{3} f\left(J_{0}+y, x, z, \bar{z}\right)=O\left(\varepsilon^{3}\right) .
\end{aligned}
$$


By the non-isocronicity property $\operatorname{det} h_{\varepsilon}^{\prime \prime}\left(J_{0}\right) \neq 0$ there is a one-to-one correspondence between the actions $J_{0} \in U \subset \mathbb{R}^{2}$ and the torus frequency $\omega:=\omega\left(J_{0}\right)=h_{\varepsilon}^{\prime}\left(J_{0}\right) \in \mathcal{O} \subset \mathbb{R}^{2}$ where

$$
\mathcal{O}:=\left\{\omega=h_{\varepsilon}^{\prime}\left(J_{0}\right): J_{0} \in U\right\} .
$$

By (105) we have

$$
\text { meas }\left(h_{\varepsilon}^{\prime}(\mathcal{I}) \backslash \mathcal{O}\right) \leq \text { const } \alpha_{0}=O\left(\sqrt{\varepsilon} \log \left(\frac{1}{\varepsilon}\right)\right) .
$$

The frequencies $\omega:=\omega\left(J_{0}\right)$ can be introduced as parameters: denoting $J_{0}=$ $J_{0}(\omega):=\left(h_{\varepsilon}^{\prime}\right)^{-1}(\omega)$ its inverse function, the Hamiltonian (110) can be finally written in the form

$$
H(y, \psi, z, \bar{z} ; \omega):=\mathcal{N}(y, z, \bar{z} ; \omega)+P(y, \psi, z, \bar{z} ; \omega)
$$

where $\mathcal{N}(y, z, \bar{z} ; \omega):=e(\omega)+\omega \cdot y+\Omega(\omega) z \bar{z}, e(\omega):=h_{\varepsilon}\left(J_{0}(\omega)\right), \Omega(\omega):=$ $\varepsilon \widetilde{\Omega}\left(J_{0}(\omega)\right)$ and the perturbation $P(y, \psi, z, \bar{z} ; \omega)$ is obtained by the one in (110) just replacing $J_{0}$ with $J_{0}(\omega):=\left(h_{\varepsilon}^{\prime}\right)^{-1}(\omega)$. Recalling (102) the Hamiltonian $H$ in (114) is real analytic in

$$
(y, \psi, z, \bar{z} ; \omega) \in U_{\widetilde{r} / 2}^{2} \times \mathbb{T}_{\widetilde{s}}^{2} \times D_{\widetilde{\rho}}^{4} \times \mathcal{O}_{\widetilde{\delta}}^{2}, \quad \text { with } \quad \widetilde{\delta}:=\operatorname{const} \sqrt{\varepsilon}
$$

(since $\omega \rightarrow J_{0}(\omega):=\left(h_{\varepsilon}^{\prime}\right)^{-1}$ is analytic).

$H$ is in a suitable form to apply the KAM theorem of [Pö1] that we rewrite in the next subsection.

\section{1. - A KAM theorem for elliptic tori}

The KAM theorem of [Pö1] applies to Hamiltonians like

$$
H:=H(y, \psi, z, \bar{z} ; \omega):=\mathcal{N}+P=e(\omega)+\omega \cdot y+\Omega(\omega) z \bar{z}+P(y, \psi, z, \bar{z} ; \omega)
$$

where $(y, \psi, z, \bar{z}) \in \mathbb{R}^{n} \times \mathbb{T}^{n} \times \mathbb{C}^{m} \times \mathbb{C}^{m}$ and $\omega \in \mathbb{R}^{n}$ is regarded as a parameter varying over a compact subset $\mathcal{O} \subseteq \mathbb{R}^{n}$.

The functions $P=P(y, \psi, z, \bar{z} ; \omega)$ and $\Omega(\omega)$ are real analytic on the complex domain $\mathcal{D}_{\bar{r}, \bar{s}} \times \mathcal{O}_{\delta}$ where $\mathcal{D}_{\bar{r}, \bar{s}}:=D_{\bar{r}^{2}}^{n} \times \mathbb{T}_{\bar{s}}^{n} \times D_{\bar{r}}^{2 m}$ and $\bar{r}, \bar{s}, \delta$ are suitable positive constants.

The size of the perturbation $P$ is measured by the following norm. Taking the Fourier-Taylor expansions

$$
P(y, \psi, z, \bar{z} ; \omega)=\sum_{\substack{\ell \in \mathbb{Z}^{n} \\ a, \bar{a} \in \mathbb{N}^{m}}} P_{a, \bar{a}, \ell}(y ; \omega) z^{a} \bar{z}^{\bar{a}} e^{\mathrm{i} \ell \cdot \psi}=\sum_{\ell \in \mathbb{Z}^{n}} P_{\ell}(y, z, \bar{z} ; \omega) e^{\mathrm{i} \ell \cdot \psi},
$$

let define $e^{(23)}$

$$
\left\||P \||_{\bar{r}, \bar{s}, \delta}:=\sum_{\ell \in \mathbb{Z}^{n}}\left|\mathbf{M} P_{\ell}\right|_{\bar{r}, \delta} e^{|\ell| \bar{s}}\right.
$$

where $\mathbf{M} P_{\ell}:=\sum_{a, \bar{a} \geq 0}\left|P_{a, \bar{a}, \ell}(y)\right| z^{a} \bar{z}^{\bar{a}}$ and $|\cdot|_{\bar{r}, \delta}$ denotes the sup-norm over the $(y, z, \bar{z}) \in D_{\bar{r}^{2}}^{n} \times D_{\bar{r}}^{2 m}$ and $\omega \in \mathcal{O}_{\delta}$. The following theorem follows from [Pö1].

${ }^{(23)}$ The relation with the usual Fourier norm (used in the Appendix) $\|P\|_{\bar{r}, \bar{s}, \delta}:=\sum_{\ell \in \mathbb{Z}^{n}}\left|P_{\ell}\right|_{\bar{r}, \delta} e^{|\ell| \bar{s}}$ is $\left\|\left|P\left\|\left.\right|_{\bar{r} / 2, \bar{s}, \delta} \leq 2^{2 m}\right\| P \|_{\bar{r}, \bar{s}, \delta}\right.\right.$ and $\left\|\left|P\left\|\left.\right|_{\bar{r}, \bar{s}, \delta} \geq 2^{2 m}\right\| P \|_{\bar{r}, \bar{s}, \delta}\right.\right.$. 
THEOREM 5.1 (Pöschel [Pö1]). Fix $\tau>n-1$. Suppose

$$
\sup _{\omega \in \mathcal{O}_{\delta}}\left|\partial_{\omega} \Omega(\omega)\right| \leq M
$$

for some $0<M<+\infty$, and that the non-resonance condition

$$
|\Omega(\omega) \cdot h| \geq \alpha, \quad \forall 1 \leq|h| \leq 2, h \in \mathbb{Z}^{n}, \quad \forall \omega \in \mathcal{O},
$$

is satisfied for some $\alpha>0$. Then, there exists a positive constant $\kappa:=\kappa(\bar{s})$ such that, if $P$ is sufficiently small,

$$
\mathcal{P}:=\left\||P \||_{\bar{r}, \bar{s}, \delta} \leq \frac{\kappa}{M+1} \alpha \bar{r}^{2} \leq \frac{\delta \bar{r}^{2}}{16},\right.
$$

then:

(i) there exist a normal form $\mathcal{N}_{*}:=e_{*}+\omega \cdot y_{*}+\Omega_{*}(\omega) z_{*} \bar{z}_{*}$, a Cantor set $\mathcal{O}(\alpha) \subset \mathcal{O}$ on which

$$
\begin{aligned}
\left|\omega \cdot \ell+\Omega_{*}(\omega) \cdot h\right| & \geq \frac{\alpha}{2\left(1+|\ell|^{\tau}\right)}, \\
\forall \ell \in \mathbb{Z}^{n},|h| & \leq 2,|\ell|+|h| \neq 0, \omega \in \mathcal{O}(\alpha),
\end{aligned}
$$

and a transformation $\mathcal{F}: \mathcal{D}_{\bar{r} / 2, \bar{s} / 2} \times \mathcal{O}(\alpha) \longrightarrow \mathcal{D}_{\bar{r}, \bar{s}} \times \mathcal{O}_{\delta}$ real analytic and symplectic for each $\omega$ and Whitney smooth in $\omega$, such that

$$
H_{*}:=H \circ \mathcal{F}=\mathcal{N}_{*}+R_{*} \quad \text { with } \quad R_{*}:=\sum_{2|k|+|a+\bar{a}| \geq 3} R_{k a \bar{a}}^{*}\left(\psi_{*}\right) y_{*}^{k} z_{*}^{a} \bar{z}_{*}^{\bar{a}} ;
$$

(ii) $\mathcal{F}$ has the following form (omitting the dependence on $\omega$ )

$$
\begin{aligned}
y & =y_{*}+Y\left(y_{*}, \psi_{*}, z_{*}, \bar{z}_{*}\right) \\
\psi & =\psi_{*}+X\left(\psi_{*}\right) \\
z & =z_{*}+Z\left(\psi_{*}, z_{*}, \bar{z}_{*}\right) \\
\bar{z} & =\bar{z}_{*}+\bar{Z}\left(\psi_{*}, z_{*}, \bar{z}_{*}\right)
\end{aligned}
$$

where

$$
Y:=\sum_{2|k|+|a+\bar{a}| \leq 2} Y_{k a \bar{a}}\left(\psi_{*}\right) y_{*}^{k} z_{*}^{a} \bar{z}_{*}^{\bar{a}}, \quad Z:=\sum_{|a+\bar{a}| \leq 1} Z_{a \bar{a}}\left(\psi_{*}\right) z_{*}^{a} \bar{z}_{*}^{\bar{a}},
$$

and, denoting by $\|\cdot\|_{*}:=\sup _{\mathcal{D}_{\bar{r} / 2, \bar{s} / 2}}|\cdot|$,

$$
\|Y\|_{*}, \quad \frac{\bar{r}^{2}}{\bar{s}}\|X\|_{*}, \quad \bar{r}\|Z\|_{*}, \quad \bar{r}\|\bar{Z}\|_{*} \leq \mathrm{const} \frac{\mathcal{P}}{\alpha} ;
$$

(iii) if $M$ is sufficiently small, i.e., if

$$
M<1 / 4,
$$

then

$$
\operatorname{meas}(\mathcal{O} \backslash \mathcal{O}(\alpha))=O\left(\alpha d^{n-1}\right)
$$

where $d$ is the exterior diameter of $\mathcal{O}$. 
By Theorem 5.1 the Hamiltonian system generated by $H$ possesses a family of $n$ dimensional elliptic invariant tori $\mathcal{T}:=\left\{(y, \psi, z)=\left(Y\left(0, \psi_{*}, 0,0\right), \psi_{*}, z\left(\psi_{*}, 0,0\right)\right)\right\}$ traveled with frequencies $\omega$, for each frequency vector in the Cantor set $\mathcal{O}_{\alpha}$. The dynamics near each torus $\mathcal{T}$ is described by the normal form (120), which in turn coincides with (1).

Let us make some comments on Theorem 5.1. Point (i) is Theorem A of [Pö1] (we have fixed the constant $\rho$ in the statement of Theorem A as $\rho:=\bar{s} / 4)$.

Concerning point (ii), formula (121) follows from Section 4 of [Pö1] (see, in particular, page 574 of [Pö1]). Formula (123) follows from Section 7 of [Pö1] (see in particular the last estimate on page 592).

Point (iii) follows from Theorem B of [Pö1]. Indeed, from (116), (117) and (124) $\mathcal{O}$ is essentially non-resonant according to the definition at page 565 of [Pö1]. For finite dimensional systems this reduces to verify only that $\mathcal{O}$ is non-resonant (see again page 565 of [Pö1]), namely

$$
\min _{\omega \in \mathcal{O}}|\ell \cdot \omega+\Omega(\omega) \cdot h| \geq \alpha
$$

for all $0<|h| \leq 2$ and $\ell \in \mathcal{K}_{h}$, the closed convex hull of the gradient set $\mathcal{G}_{h}:=\left\{\partial_{\omega}(\Omega(\omega) \cdot h) \quad: \omega \in \mathcal{O}\right\}$. Since, by (116) and $|h| \leq 2,{ }^{(24)} \mathcal{K}_{h} \subseteq B_{2 M}^{n}$, taking $M<1 / 4$ as in (124), the unique integer vector $l \in \mathcal{K}_{h}$ is $l=0$. Hence condition (126) must be verified for $\ell=0$ only, and this is condition (117).

\section{2. - Abundance of periodic solutions in the three-body problem}

In this section we prove the existence of periodic orbits accumulating on elliptic 2-dimensional tori of the three-body problem.

First of all we show that the KAM-Theorem 5.1 applies to the Hamiltonian $H$ in (114) reproving the existence of elliptic invariant tori in the spatial planetary three body problem as in $[\mathrm{BCV}]$. We assume the frequency parameter $\omega \in \mathbb{R}^{2}$ to vary over the compact subset $\mathcal{O}$.

By (115) and (102), the Hamiltonian $H$ in (114) is real analytic on $\mathcal{D}_{\bar{r}, \bar{s}} \times$ $\mathcal{O}_{\delta}:=D_{\bar{r}^{2}}^{2} \times \mathbb{T}_{\bar{s}}^{2} \times D_{\bar{r}}^{4} \times \mathcal{O}_{\delta}$ for

$$
\bar{r}:=\sqrt{c_{0} \varepsilon}, \quad \bar{s}:=\text { const }, \quad \delta:=\operatorname{const} \sqrt{\varepsilon},
$$

where $c_{0}$ is a small constant which will be determined later on (we restrict the domain of $H$ w.r.t. to the larger one defined in (115) because with the choice (127) the smallness KAM condition (118) is satisfied, see (132), (133)).

Applying the KAM-Theorem 5.1, we get

THEOREM 5.2. Fix $\tau>n-1$. For $c_{0}$ and $\varepsilon$ small enough, there exists a Cantor set $\mathcal{O}(\varepsilon) \subset \mathcal{O} \subset h_{\varepsilon}^{\prime}(\mathcal{I})$, with

$$
\operatorname{meas}\left(h_{\varepsilon}^{\prime}(\mathcal{I}) \backslash \mathcal{O}(\varepsilon)\right) \leq \operatorname{const} \sqrt{\varepsilon} \log \frac{1}{\varepsilon},
$$

${ }^{(24)} B_{R}^{n} \subset \mathbb{R}^{n}$ is the closed ball of radius $R$ and center 0 using the $|\cdot|_{2}$ norm. 
such that, for any $\omega=\omega\left(J_{0}\right) \in \mathcal{O}(\varepsilon)$, there exists a symplectic transformation

$$
\Phi: \mathcal{D}_{\bar{r} / 2, \bar{s} / 2} \longrightarrow \mathcal{D}_{\bar{r}, \bar{s}}
$$

of the form (121), transforming the three-body Hamiltonian $H$ in (114) into the normal form $H_{*}:=H \circ \Phi$ as in (120). Moreover

$$
\begin{aligned}
&\left|\omega \cdot \ell+\Omega_{*}(\omega) \cdot h\right| \geq \frac{\text { const } \varepsilon}{1+|\ell|^{\tau}}, \\
& \forall \ell \in \mathbb{Z}^{n},|h| \leq 2,|\ell|+|h| \neq 0, \omega \in \mathcal{O}(\varepsilon) .
\end{aligned}
$$

In particular, the three-body problem Hamiltonian $H$ possesses a family of 2dimensional elliptic invariant tori traveled with frequencies $\omega$.

Proof. Recalling that $\Omega(\omega):=\varepsilon \widetilde{\Omega}\left(J_{0}(\omega)\right)$, it is easy to see that $\sup _{\omega \in \mathcal{O}_{\delta}}\left|\partial_{\omega} \Omega(\omega)\right| \leq \varepsilon C_{0}:=M$ for some positive constant $C_{0}$ and condition (116) holds true. Moreover, for $0<\varepsilon<1 /\left(4 C_{0}\right), M<1 / 4$ and also condition (124) is satisfied.

We claim that condition (117) holds, with

$$
\alpha:=\chi_{0} \varepsilon
$$

namely,

$$
|\Omega(\omega) \cdot h| \geq \alpha:=\chi_{0} \varepsilon, \quad \forall 1 \leq|h| \leq 2, h \in \mathbb{Z}^{2}, \quad \forall \omega \in \mathcal{O}
$$

Indeed $\forall \omega \in \mathcal{O}, \Omega(\omega)=\varepsilon \widetilde{\Omega}\left(J_{0}\right)$ for some $J_{0} \in U$. Moreover, by (107), it is easy to see that $\left|\widetilde{\Omega}\left(J_{0}\right) \cdot h\right| \geq \chi_{0}, \forall 1 \leq|h| \leq 2, h \in \mathbb{Z}^{2}, J_{0} \in U$ and the claim follows.

It remains to check the smallness condition (118), i.e. $\mathcal{P} \leq \varepsilon \bar{r}^{2}$, since $\kappa=O(1)$ and $\alpha:=\chi_{0} \varepsilon$. Note that, from (111) and (127),

$$
\mathcal{P} \leq \operatorname{const}\left(\bar{r}^{4}+\varepsilon \bar{r}^{4}+\varepsilon \bar{r}^{3}+\varepsilon^{3}\right) \leq \operatorname{const}\left(\bar{r}^{4}+\varepsilon^{3}\right) .
$$

Hence, by (132), in order to check condition (118), it is sufficient that

$$
\bar{r}^{4}+\varepsilon^{3} \leq \text { const } \varepsilon \bar{r}^{2}
$$

Since $\bar{r}:=\sqrt{c_{0} \varepsilon}$, (133) holds true for $c_{0}$ and $\varepsilon$ small enough. By Theorem 5.1 the result follows.

From (125), since $d=O(1)$ and $\alpha=O(\varepsilon)$, meas $(\mathcal{O} \backslash \mathcal{O}(\varepsilon))=O(\varepsilon)$. Moreover, since by $(113)$, meas $\left(h_{\varepsilon}^{\prime}(\mathcal{I}) \backslash \mathcal{O}\right)=O(\sqrt{\varepsilon} \log (1 / \varepsilon))$, the measure estimate (128) follows. 
For proving that each elliptic invariant torus found in Theorem 5.2 lies in the closure of periodic orbits of the three body problem, we will apply Theorem 1.1 to the normal form Hamiltonian $H_{*}:=H \circ \Phi$ given in (120) (which has the form (1)) corresponding to the three body problem.

The crucial hypothesis to verify in Theorem 1.1 is the non-degeneracy of the twist matrix $\mathcal{R}$ defined in (7). We have to evaluate, for $1 \leq i, i^{\prime} \leq n=2$ and $1 \leq j \leq m=2$

$$
\begin{aligned}
R_{e_{i}+e_{i^{\prime}}, 0,0}^{*}\left(\psi_{*}\right) & =\frac{1}{1+\delta_{i, i^{\prime}}} \frac{\partial^{2} H_{*}}{\partial y_{* i} \partial y_{* i^{\prime}}}\left(0, \psi_{*}, 0,0\right), \\
R_{e_{i}, e_{j}, 0}^{*}\left(\psi_{*}\right) & =\frac{\partial^{2} H_{*}}{\partial y_{* i} \partial z_{* j}}\left(0, \psi_{*}, 0,0\right) .
\end{aligned}
$$

$R_{e_{i}, 0, e_{j}}^{*}\left(\psi_{*}\right)$ can be computed as the $R_{e_{i}, e_{j}, 0}^{*}\left(\psi_{*}\right)$ and their calculation is omitted. Due to the special form (121)-(122) of the canonical transformation $\Phi$, we have

$$
\partial_{y_{* i}}(H \circ \Phi)=\left[\left(\partial_{y} H\right) \circ \Phi\right] \partial_{y_{* i}} y \quad \text { with } \quad \partial_{y_{* i}} y=e_{i}+Y_{e_{i}, 0,0}\left(\psi_{*}\right)
$$

from which

$$
\begin{aligned}
\partial_{y_{* i} y_{* i}}^{2}(H \circ \Phi)= & {\left[\left(\partial_{y y}^{2} H\right) \circ \Phi \partial_{y_{* i}^{\prime}} y\right] \partial_{y_{* i}} y } \\
\partial_{z_{* j} y_{* i}}^{2}(H \circ \Phi)= & {\left[\left(\partial_{y y}^{2} H\right) \circ \Phi \partial_{z_{* j}} Y+\left(\partial_{z y}^{2} H\right) \circ \Phi\left(e_{j}+\partial_{z_{* j}} Z\right)\right.} \\
& \left.+\left(\partial_{\bar{z} y}^{2} H\right) \circ \Phi \partial_{z_{* j}} \bar{Z}\right] \partial_{y_{* i}} y
\end{aligned}
$$

We are now able to prove that

Lemma 5.1. For $c_{0}$ and $\varepsilon$ small enough, the twist matrix $\mathcal{R}$ of the planetary three body problem is invertible and $\left|\mathcal{R}^{-1}\right|=O(1)$.

Proof. We need to evaluate (136)-(137) for $\left(y_{*}, \psi_{*}, z_{*}, \bar{z}_{*}\right)=\left(0, \psi_{*}, 0,0\right)=$ : . By (121), (123), (132) and since $r_{0}:=\sqrt{c_{0} \varepsilon}$ and $\alpha:=\chi_{0} \varepsilon$, it results

(138) $\left|y_{i}(\star)\right|=\left|Y_{i}(\star)\right| \leq$ const $\frac{\mathcal{P}}{\alpha} \leq$ const $\frac{\bar{r}^{4}+\varepsilon^{3}}{\varepsilon}=\operatorname{const} \frac{c_{0}^{2} \varepsilon^{2}+\varepsilon^{3}}{\varepsilon} \leq$ const $\varepsilon$.

Moreover, by (123) and standard "Cauchy estimates"(25), we have

$$
\left|\partial_{y_{*}} Y(\star)\right|, \quad \frac{1}{\bar{r}}\left|\partial_{z *} Y(\star)\right|, \quad\left|\partial_{z *} Z(\star)\right|, \quad\left|\partial_{z *} \bar{Z}(\star)\right| \leq \mathrm{const} \frac{\mathcal{P}}{\alpha \bar{r}^{2}}=O\left(c_{0}\right),
$$

(for the second estimate note that $\partial_{z_{*}} Y$ is independent of $y_{*}$ due to (122)).

${ }^{(25)}$ Cauchy estimates allow to bound $n$-derivatives of analytic functions on a set $A$ in terms of their sup-norm on larger domains $A \subset A^{\prime}$ divided by $\operatorname{dist}\left(\partial A, \partial A^{\prime}\right)^{n}$. 
From (136)-(137) we deduce, using (138), (139) and since $H=h_{0}\left(J_{0}+\right.$ $y)+O(\varepsilon)$, with $h_{0}$ defined in (97),

$$
\begin{aligned}
& \partial_{y_{* i} y_{* i}}^{2} H_{*}(\star)=\frac{-3 \kappa_{i}}{\left(\left(J_{0}\right)_{i}+y_{i}(\star)\right)^{4}}+O_{\sharp}\left(\frac{\mathcal{P}}{\alpha \bar{r}^{2}}\right)+O_{\sharp}(\varepsilon)=\frac{-3 \kappa_{i}}{\left(J_{0}\right)_{i}^{4}}+O_{\sharp}\left(c_{0}\right), \\
& \partial_{y_{* i} y_{* i}}^{2} H_{*}(\star)=O_{\sharp}\left(c_{0}\right)+O_{\sharp}(\varepsilon)=O_{\sharp}\left(c_{0}\right) \quad \text { if } \quad i \neq i^{\prime}, \\
& \partial_{z_{* j} y_{* i}}^{2} H_{*}(\star),=O_{\sharp}\left(\frac{\mathcal{P}}{\alpha \bar{r}}\right)+O_{\sharp}(\varepsilon)=O_{\sharp}\left(c_{0}^{3 / 2} \sqrt{\varepsilon}\right),
\end{aligned}
$$

where $O_{\sharp}(c)$ denotes a function of $\psi_{*}$ with sup-norm for $\psi_{*} \in \mathbb{T}_{\bar{s} / 2}$ smaller than a constant multiplied by $c$. It follows

$$
\begin{gathered}
R_{2 e_{i}, 0,0}^{*}\left(\psi_{*}\right)=\frac{-3 \kappa_{i}}{2\left(J_{0}\right)_{i}^{4}}+O_{\sharp}\left(c_{0}\right), \quad R_{e_{i}+e_{i^{\prime}}, 0,0}^{*}\left(\psi_{*}\right)=O_{\sharp}\left(c_{0}\right) \\
\text { if } i^{\prime} \neq i, \quad R_{e_{i}, e_{j}, 0}^{*}\left(\psi_{*}\right)=O_{\sharp}\left(c_{0}^{3 / 2} \sqrt{\varepsilon}\right) .
\end{gathered}
$$

Evaluating the Fourier coefficients of the above function we obtain

$$
\begin{array}{ll}
\text { (140) } \quad R_{2 e_{i}, 0,0,0}^{*}=\frac{-3 \kappa_{i}}{2\left(J_{0}\right)_{i}^{4}}+O\left(c_{0}\right), & R_{e_{i}+e_{i^{\prime}}, 0,0,0}^{*}=O\left(c_{0}\right), \text { for } i^{\prime} \neq i \\
\text { (141) }\left|R_{e_{i}, e_{j}, 0, \ell}^{*}\right|, & \left|R_{e_{i}, 0, e_{j}, \ell}^{*}\right| \leq \text { const } c_{0}^{3 / 2} \sqrt{\varepsilon} e^{-|\ell| s / 4} .
\end{array}
$$

From (129), $\left|\omega \cdot \ell+\Omega_{* j}(\omega)\right| \geq$ const $\varepsilon /\left(1+|\ell|^{\tau}\right), \forall \ell \in \mathbb{Z}^{n}$ and then, from (141) the second addendum in definition of the twist matrix $\mathcal{R}$ introduced in (7) is

$$
\begin{aligned}
& \sum_{\substack{1 \leq j \leq m \\
\ell \in \mathbb{Z}^{n}}} \frac{1}{\omega \cdot \ell+\Omega_{* j}(\omega)}\left(R_{e_{i}, e_{j}, 0, \ell}^{*} R_{e_{i^{\prime}}, 0, e_{j},-\ell}^{*}+R_{e_{i}, 0, e_{j},-\ell}^{*} R_{e_{i^{\prime}, e_{j}, 0, \ell}}^{*}\right) \\
& =O\left(\sum_{\ell \in \mathbb{Z}^{n}} \frac{1+|\ell|^{\tau}}{\varepsilon} c_{0}^{3} \varepsilon e^{-\frac{|\ell| \bar{s}}{2}}\right)=O\left(c_{0}^{3}\right) .
\end{aligned}
$$

Finally (140) and (142) imply that the entries of the twist matrix $\mathcal{R}$ corresponding to the spatial three-body problem are

$$
\mathcal{R}_{i, i}=\frac{-3 \kappa_{i}}{2\left(J_{0}\right)_{i}^{4}}+O\left(c_{0}\right)+O\left(c_{0}^{3}\right), \quad \mathcal{R}_{i, i^{\prime}}=O\left(c_{0}\right)+O\left(c_{0}^{3}\right) \text { for } i^{\prime} \neq i
$$

By (143), for $c_{0}$ small enough, the matrix $\mathcal{R}$ is invertible and $\left|\mathcal{R}^{-1}\right|=O$ (1).

We can finally prove the abundance of periodic solutions in the spatial planetary three body problem.

Proof of Theorem 0.2. Since the number of elliptic variables $z$ is $m=2$ condition (a)-(i) of Theorem 1.1 holds. Moreover, by Lemma 5.1, the twist matrix $\mathcal{R}$ is invertible with $\left|\mathcal{R}^{-1}\right|=O(1)$, and then we can apply Theorem 1.1 , proving the existence of an abundance of periodic solutions of the three body problem. Theorem 0.2 is finally proved. 


\section{6. - Periodic orbits near resonant elliptic tori}

In this section we study the persistence, for $\varepsilon>0$, of the circular decoupled periodic motions of the planets around the star, once suitable conditions on the period and the masses of the bodies are satisfied.

Let consider $J_{0} \in \mathcal{I}, T>0$ and $k=\left(k_{1}, k_{2}\right) \in \mathbb{Z}^{2}$ with $\operatorname{gcd}\left(k_{1}, k_{2}\right)=1$, such that

$$
\omega:=h_{0}^{\prime}\left(J_{0}\right)=\frac{2 \pi k}{T},
$$

where $h_{0}$ is the integrable Hamiltonian defined in (97). The decoupled threebody problem possesses the family of (circular) periodic solutions

$$
\check{\zeta}_{\psi_{0}}(t):=\left(J_{0}, \psi_{0}+\omega t, 0,0\right),
$$

with minimal period $T$ and parametrized by $\psi_{0} \in \mathbb{T}^{2}$.

Since $h_{0}$ is properly degenerate the persistence of these motions for $\varepsilon>0$ can not be established without further informations on the perturbation $f_{0}$. For continuing some of these solutions we will exploit the normal form $\mathcal{H}$ defined in (101) (with $N=4$ ).

First of all, through a Lyapunov-Schmidt reduction similar to the one of Lemma 4.3 , we obtain the existence of suitable pseudo- $T$-periodic solutions.

Lemma 6.1. There exists $c_{1}, \varepsilon_{1}>0$, such that, for all $T>0, k \in \mathbb{Z}^{2}$ with $\operatorname{gcd}\left(k_{1}, k_{2}\right)=1,0<\varepsilon \leq \min \left\{\varepsilon_{1}, \frac{1}{c_{1} T}\right\}, J_{0} \in U$ satisfying $\omega:=h_{0}^{\prime}\left(J_{0}\right)=2 \pi k / T$, and $\forall \psi_{0} \in \mathbb{T}^{2}$, there exists a unique function

$$
\zeta_{\psi_{0}}=\left(J_{\psi_{0}}, \psi_{\psi_{0}}, z \psi_{0}\right) \in C^{0}\left([0, T], \mathbb{R}^{2} \times \mathbb{T}^{2} \times \mathbb{C}^{2}\right) \cap C^{1}\left((0, T), \mathbb{R}^{2} \times \mathbb{T}^{2} \times \mathbb{C}^{2}\right),
$$

smooth in $\psi_{0}$, so that, $\forall t \in(0, T), \zeta_{\psi_{0}}(t)$ is a solution of the Hamiltonian system $(109)^{(26)}$ with $\psi_{\psi_{0}}(0)=\psi_{\psi_{0}}(T)=\psi_{0}, z_{\psi_{0}}(0)=z_{\psi_{0}}(T)$ and

$$
\sup _{t \in[0, T]}\left|\zeta_{\psi_{0}}(t)-\hat{\zeta}_{\psi_{0}}(t)\right| \leq c_{1} \varepsilon^{2}
$$

where $\hat{\zeta}_{\psi_{0}}(t):=\left(J_{\varepsilon}, \psi_{0}+\omega t, 0,0\right)$ and $J_{\varepsilon}=J_{0}+O(\varepsilon)$ is the unique solution of $h_{\varepsilon}^{\prime}\left(J_{\varepsilon}\right)=\omega$.

Proof. We first note that, since $\operatorname{det} h_{0}^{\prime \prime}(J) \neq 0$ (see (96)-(97)), by the Implicit Function Theorem, $J_{\varepsilon}$ exists, is unique and is $\varepsilon$-close to $J_{0} \in U$. Moreover, for $\varepsilon_{1}$ small enough, $J_{\varepsilon} \in \mathbb{R}^{2} \cap U_{\text {const } \varepsilon} \subset U_{\widetilde{r} / 4}$ (since $\widetilde{r}=O(\sqrt{\varepsilon})$ ).

Define $X$ and $Y$ as in Lemma 2.5, with $n=m=2$. Let also $\Omega:=\varepsilon \widetilde{\Omega}\left(J_{\varepsilon}\right)$, $M:=\partial_{J}^{2} h_{\varepsilon}\left(J_{\varepsilon}\right)$ and $\mathcal{M}:=\mathbf{1}_{2}-e^{\mathrm{i} \varepsilon \widetilde{\Omega}\left(J_{\varepsilon}\right) T}$. Notice that, if $0<\varepsilon T \leq 1 / c_{1}$ is

${ }^{(26)}$ For the Hamiltonian $\mathcal{H}$ defined in (101) with $N=4$. 
small enough (namely $c_{1}$ is large enough), then $\left|\mathcal{M}^{-1}\right|$ is bounded by a constant (independent on $\varepsilon$ ).

We look for $T$-periodic solutions of the Hamiltonian system (109) of the form $\zeta=\hat{\zeta}_{\psi_{0}}+(\widetilde{J}, \widetilde{\psi}, \widetilde{z}, \widetilde{\bar{z}})$. Set

$$
P\left(\begin{array}{c}
\widetilde{J} \\
\widetilde{\psi} \\
\widetilde{z}
\end{array}\right)=\left(\begin{array}{c}
-\varepsilon^{4} \partial_{\psi} f(\star) \\
-\partial_{J}^{2} h_{\varepsilon}\left(J_{\varepsilon}\right) \widetilde{J}+\partial_{J} h_{\varepsilon}\left(J_{\varepsilon}+\widetilde{J}\right)-\partial_{J} h_{\varepsilon}\left(J_{\varepsilon}\right)+\varepsilon \partial_{J} \widetilde{\Omega}\left(J_{\varepsilon}+\widetilde{J}\right) \widetilde{z} \cdot \bar{z}+ \\
+\left(\varepsilon \partial_{J} g+\varepsilon^{4} \partial_{J} f\right)(\star) \\
\mathrm{i}\left[\varepsilon\left(\widetilde{\Omega}\left(J_{\varepsilon}+\widetilde{J}\right)-\widetilde{\Omega}\left(J_{\varepsilon}\right)\right) \cdot \widetilde{z}+\left(\varepsilon \partial_{z} g+\varepsilon^{4} \partial_{\bar{z}} f\right)(\star)\right]
\end{array}\right),
$$

where the star above denotes, for short, $\star:=\left(J_{\varepsilon}+\widetilde{J}, \psi_{0}+\omega t+\widetilde{\psi}, \widetilde{z}, \widetilde{z}\right)$. We note that $P(0)=O\left(\varepsilon^{4}\right)$ by (106). We want to apply Lemma 2.4. From (25) of Lemma 2.5 we find $|L|=O\left(1 /\left(c_{1}^{2} \varepsilon^{2}\right)\right)$, hence $\delta_{0}:=2|L(P(0))|=O\left(\varepsilon^{2} / c_{1}^{2}\right)$ and $\sup _{x \in B_{\delta_{0}}}|D P(x)| \leq \operatorname{const}\left(\delta_{0}+\varepsilon^{4}\right)$. Then, taking $c_{1}$ big enough, we can apply Lemma 2.4 proving the existence and uniqueness of a $\zeta_{\psi_{0}}^{\star} \in B_{\rho_{0}}$ so that

$$
\zeta_{\psi_{0}}^{\star}=L\left(P\left(\zeta_{\psi_{0}}^{\star}\right)\right)
$$

Let $\zeta_{\psi_{0}}(t):=\hat{\zeta}_{\psi_{0}}+\zeta_{\psi_{0}}^{\star}$. By means of (145) and (26), it follows that $\zeta_{\psi_{0}}$ satisfies the Hamilton equations (109) in $(0, T)$ and the boundary conditions $\psi_{\psi_{0}}(0)=\psi_{\psi_{0}}(T)=\psi_{0}, z_{\psi_{0}}(0)=z_{\psi_{0}}(T)$. As usual, by the Implicit Function Theorem, $\psi_{0} \rightarrow \zeta_{\psi_{0}}$ is smooth.

Finally, critical points of the "reduced action functional" $\mathcal{E}: \mathbb{T}^{2} \rightarrow \mathbb{R}$ defined by

$$
\mathcal{E}\left(\psi_{0}\right):=\int_{0}^{T} J_{\psi_{0}} \cdot \dot{\psi}_{\psi_{0}}+\mathrm{i} z_{\psi_{0}} \cdot \dot{\bar{z}}_{\psi_{0}}-\mathcal{H}\left(\zeta_{\psi_{0}}\right) d t
$$

give rise, arguing as in Lemma 4.4, to periodic solutions of (109). As in the proof of Theorem 1.1 (see page 117) we deduce the existence of at least two geometrically distinct periodic solutions of (109) (corresponding to the points of maximum $\psi_{0}^{+}$and minimum $\psi_{0}^{-}$of $\mathcal{E}$ ).

We can finally state the following result which, in particular, will imply Theorems 0.3 and 0.4 :

THEOREM 6.1. There exists $c_{1}, \varepsilon_{1}>0$, such that, for all $T>0, k \in \mathbb{Z}^{2}$ with $\operatorname{gcd}\left(k_{1}, k_{2}\right)=1,0<\varepsilon \leq \min \left\{\varepsilon_{1}, \frac{1}{c_{1} T}\right\}, J_{0} \in U$ satisfying $\omega:=h_{0}^{\prime}\left(J_{0}\right)=$ $2 \pi k / T$, there exist at least two geometrically distinct $T$-periodic solutions $\zeta_{\psi_{0}^{ \pm}}=$ $\left(J_{\psi_{0}^{ \pm}}, \psi_{\psi_{0}^{ \pm}}, z_{\psi_{0}^{ \pm}}\right)$of the Hamiltonian system $(109)^{(27)}$ with

$$
\sup _{t \in \mathbb{R}}\left|\zeta_{\psi_{0}^{ \pm}}(t)-\hat{\zeta}_{\psi_{0}^{ \pm}}(t)\right| \leq c_{1} \varepsilon^{2}
$$

where $\hat{\zeta}_{\psi_{0}^{ \pm}}(t):=\left(J_{\varepsilon}, \psi_{0}^{ \pm}+\omega t, 0,0\right)$ and $J_{\varepsilon}=J_{0}+O(\varepsilon)$ is the unique solution of $h_{\varepsilon}^{\prime}\left(J_{\varepsilon}\right)=\omega=2 \pi k / T$. Note that $\sup _{t \in[0, T]}\left|\zeta_{\psi_{0}^{ \pm}}(t)-\left(J_{0}, \psi_{0}^{ \pm}+\omega t, 0,0\right)\right|=O(\varepsilon)$.

${ }^{(27)}$ For the Hamiltonian $\mathcal{H}$ defined in (101) with $N=4$. 
REMARK 6.1. The condition $0<\varepsilon T<1 / c_{1}$ (for $c_{1}$ large enough) required in the theorem is sharp. Indeed, for $\varepsilon T=O(1)$ some further resonance phenomenon can appear, destroying any periodic solution: note that $e^{\mathrm{i} \varepsilon \widetilde{\Omega}\left(J_{\varepsilon}\right) T}-\mathbf{1}_{2}$ could be degenerate. It is for reaching this estimate that we take $N=4$ in the Hamiltonian (101).

We now show that Theorems 0.3 and 0.4 follow from Theorem 6.1.

Consider $J_{0} \in \mathcal{I}, T>0, k=\left(k_{1}, k_{2}\right) \in \mathbb{Z}^{2}$ with $\operatorname{gcd}\left(k_{1}, k_{2}\right)=1$, such that $\omega:=h_{0}^{\prime}\left(J_{0}\right)=2 \pi k / T$, i.e. (144) holds. Under suitable restrictions on $\varepsilon$ and $T$ we shall prove that $J_{0} \in U$ where $U=U(\varepsilon) \subset \mathcal{I}$ is the domain defined in (103).

First of all we claim that there exists a constant $c_{2}>0$ large enough such that

$$
\varepsilon \geq e^{-T / c_{2}} \Longrightarrow \omega \cdot \ell \neq 0, \quad \forall 0<|\ell| \leq K
$$

where $K=K(\varepsilon)=$ const $\log (1 / \varepsilon)$ is defined in (104). In fact, since $\operatorname{gcd}\left(k_{1}, k_{2}\right)=1$,

$$
\omega \cdot \ell=0 \quad \Longrightarrow \quad k \cdot \ell=0 \quad \Longrightarrow \quad \ell \in \mathbb{Z}\left(-k_{2}, k_{1}\right) \Longrightarrow \quad|\ell| \geq|k| .
$$

Moreover

$$
|k|=T \frac{\left|h_{0}^{\prime}\left(J_{0}\right)\right|}{2 \pi} \geq c_{\mathcal{I}} T
$$

where $c_{\mathcal{I}}:=(1 / 2 \pi) \min _{J_{0} \in \mathcal{I}}\left|h_{0}^{\prime}\left(J_{0}\right)\right|>0$ (note that $h_{0}^{\prime}\left(J_{0}\right)=\left(\kappa_{1} J_{01}^{-3}, \kappa_{2} J_{02}^{-3}\right) \neq$ 0 and $\mathcal{I}$ is compact).

From (147)-(148) and the hypothesis $\varepsilon \geq e^{-T / c_{2}}$, we obtain that $k \cdot \ell=0$, $\ell \neq 0$, imply $|\ell| \geq c_{\mathcal{I}} T>c_{\mathcal{I}} c_{2} \log (1 / \varepsilon)$. For $c_{2}$ large enough, $c_{\mathcal{I}} c_{2} \log (1 / \varepsilon)>$ $K=$ const $\log (1 / \varepsilon)$, and we conclude that $k \cdot \ell \neq 0$ for all $0<|\ell| \leq K$. The claim is proved.

It follows that $\forall 0<|\ell| \leq K, k \cdot \ell \geq 1$ and hence $|\omega \cdot \ell|=2 \pi|k \cdot \ell| T^{-1} \geq$ $2 \pi T^{-1}$. Moreover, if $\varepsilon \log ^{2}(1 / \varepsilon) \leq c_{3} T^{-2}$, then $2 \pi T^{-1} \geq 2 \pi \sqrt{\varepsilon / c_{3}} \log (1 / \varepsilon) \geq$ $\alpha=$ const $\sqrt{\varepsilon} \log (1 / \varepsilon)$ (the constant $\alpha$ is defined in (104)) for a suitable $c_{3}>0$ large enough.

We have proved that conditions $\varepsilon \geq e^{-T / c_{2}}$ and $\varepsilon \log ^{2}(1 / \varepsilon) \leq c_{3} T^{-2}$ imply $J_{0} \in U$. In conclusion, defining the functions

$$
\begin{aligned}
\underline{\varepsilon}(T) & :=\min \left\{e^{-T / c_{2}}, \quad\left(c_{1} T\right)^{-1}, \varepsilon_{1}\right\} \\
\text { and } \quad \bar{\varepsilon}(T) & :=\min \left\{F\left(c_{3} T^{-2}\right), \quad\left(c_{1} T\right)^{-1}, \quad \varepsilon_{1}\right\}
\end{aligned}
$$

where $F$ is the inverse of $G(\varepsilon):=\varepsilon \log ^{2}(1 / \varepsilon)$ and $c_{1}, \varepsilon_{1}$ are defined in Lemma 6.1 , we have proved: 
LeMmA 6.2. Let $T>0, k=\left(k_{1}, k_{2}\right) \in \mathbb{Z}^{2}$, with $\operatorname{gcd}\left(k_{1}, k_{2}\right)=1$, and $J_{0} \in \mathcal{I}$ such that $\omega:=h_{0}^{\prime}\left(J_{0}\right)=\frac{2 \pi k}{T}$ holds. Then

$$
\underline{\varepsilon}(T) \leq \varepsilon \leq \bar{\varepsilon}(T) \quad \Longrightarrow \quad J_{0} \in U
$$

We can finally deduce Theorems 0.3 and 0.4 .

Proof of TheOREM 0.3. It is a direct consequence of Lemma 6.2 and of Theorem 6.1 taking $\underline{\varepsilon}(T), \bar{\varepsilon}(T)$ as in (149) and $T_{0}>0$ as the last instant for which $\underline{\varepsilon}\left(T_{0}\right)=\bar{\varepsilon}\left(T_{0}\right)$.

Proof of Theorem 0.4. Define

$$
\underline{T}(\varepsilon):=\min \left\{c_{2} \log \frac{1}{\varepsilon}, \frac{1}{c_{1} \varepsilon_{1}}\right\} \quad \text { and } \quad \bar{T}(\varepsilon):=\min \left\{\frac{\sqrt{c_{3}}}{\sqrt{\varepsilon} \log (1 / \varepsilon)}, \frac{1}{c_{1} \varepsilon_{1}}\right\}
$$

with $\varepsilon_{1}, c_{2}, c_{3}$ defined above. Theorem 0.4 is a direct consequence of Lemma 6.2 and of Theorem 6.1.

\section{7. - Appendix: Proof of Proposition 5.1}

We first recall the following Averaging Theorem, the proof of which can be found in [BCV]. We first introduce some notations. For $r, \rho, s>0$ and $U \subset \mathbb{R}^{2}$ we denote the complex set $W_{r, \rho, s}:=U_{r} \times \mathbb{T}^{2} \times D_{\rho}^{4}$. For a function $f:=f(I, \varphi, p, q)$ real analytic for $(I, \varphi, p, q) \in W_{r, \rho, s}$ we denote by $\|f\|_{r, \rho, s}$ its "sup-Fourier" norm given by

$$
\|f\|_{r, \rho, s}:=\sum_{k \in \mathbb{Z}^{2}}\left(\sup _{(I, p, q) \in U_{r} \times D_{\rho}^{4}}\left|f_{k}(I, p, q)\right|\right) e^{|k| s} .
$$

Proposition 7.1 Averaging Theorem. Let $\mathcal{H}_{0}:=h_{0}\left(I_{0}\right)+f_{0}\left(I_{0}, \varphi_{0}, p_{0}, q_{0}\right)$ be a real-analytic Hamiltonian on $W_{r, \rho, s}$. Assume that there exist $\alpha_{0}, K>0$, satisfying $K s \geq 6$, such that

$$
\left|h_{0}^{\prime}\left(I_{0}\right) \cdot k\right| \geq \alpha_{0} / 2, \quad \forall k \in\left\{k \in \mathbb{Z}^{2}: 0<|k| \leq K\right\}, \quad \forall I_{0} \in U_{r}
$$

Assume also that, if $d:=\min \left\{r s, \rho^{2}\right\}$, then

$$
\left\|f_{0}\right\|_{r, \rho, s}<\frac{\alpha_{0} d}{c K s}
$$


where $c>1$ is a suitable universal constant ${ }^{(28)}$. Then, there exists a real-analytic symplectic transformation

$$
\Psi:\left(I^{\prime}, \varphi^{\prime}, p^{\prime}, q^{\prime}\right) \in W_{r / 2, \rho / 2, s / 6} \mapsto\left(I_{0}, \varphi_{0}, p_{0}, q_{0}\right)=\Psi\left(I^{\prime}, \varphi^{\prime}, p^{\prime}, q^{\prime}\right) \in W_{r, \rho, s}
$$

and a real-analytic function $g_{0}=g_{0}\left(I^{\prime}, p^{\prime}, q^{\prime}\right)$ such that

$$
\mathcal{H}^{\prime}:=\mathcal{H}_{0} \circ \Psi=h_{0}+g_{0}+f_{*},
$$

and the following bounds hold ${ }^{(29)}$ :

$$
\sup _{\left(I^{\prime}, p^{\prime}, q^{\prime}\right) \in U_{r / 2} \times V_{\rho / 2}}\left|g_{0}\left(I^{\prime}, p^{\prime}, q^{\prime}\right)-f_{0,0}\left(I^{\prime}, p^{\prime}, q^{\prime}\right)\right| \leq \frac{c}{\alpha_{0} d}\|f\|_{r, \rho, s}^{2},
$$

Furthermore, for each $\left(I^{\prime}, \varphi^{\prime}, p^{\prime}, q^{\prime}\right) \in W_{r / 2, \rho / 2, s / 6},\left(I_{0}, \varphi_{0}, p_{0}, q_{0}\right)=\Psi\left(I^{\prime}, \varphi^{\prime}, p^{\prime}, q^{\prime}\right)$ satisfies

$$
s\left|I_{0}-I^{\prime}\right|, \quad r\left|\varphi_{0}-\varphi^{\prime}\right|, \quad \rho\left|p_{0}-p^{\prime}\right|, \quad \rho\left|q_{0}-q^{\prime}\right| \leq \frac{c\|f\|_{r, \rho, s}}{\alpha_{0}} .
$$

Let, now, $\mathcal{H}_{0}=h_{0}+f_{0}$ be as in (96). and $U$ as in (103). The estimate (105) directly follows by the definition of $U$. Next, let us choose the sets and the parameters involved in Proposition 7.1 as follows:

$$
\begin{gathered}
K:=\frac{6(N-1)}{s_{0}} \log \frac{1}{\varepsilon}, \quad \alpha_{0}:=2 \sup _{\mathcal{I}_{\sigma_{0}}}\left|h_{0}^{\prime \prime}\right| r K, \\
r=c_{*} \sqrt{\varepsilon} \leq \sigma_{0}, \quad s:=s_{0}, \quad \rho:=\rho_{0},
\end{gathered}
$$

where $c_{*}$ is a suitable large constant to be fixed later and $\sigma_{0}, s_{0}, \rho_{0}$ were defined in (98). Moreover we better specify the definition of $U$ in (103):

$$
U:=\left\{J \in \mathcal{I} \quad: \quad\left|h_{0}^{\prime}(J) \cdot \ell\right| \geq \alpha_{0}, \forall \ell \in \mathbb{Z}^{2}, 0<|\ell| \leq K\right\} \subset \mathbb{R}^{2} .
$$

The estimate (105) directly follows by the previous definition. Notice that, from these definitions, there follows (for $\varepsilon$ small enough) that

$$
\alpha_{0}=\text { const } \sqrt{\varepsilon} \log \frac{1}{\varepsilon}, \quad d=\text { const } r, \quad \frac{\alpha_{0} d}{c K s}=\operatorname{const} \varepsilon c_{*}^{2},
$$

(clearly, in the last evaluation, "const" does not involve $c_{*}$ ).

${ }^{(28)}$ Explicitly, one can take, for instance, $c=2^{9} e^{2}$, see Proposition A.1 in [BCV]

${ }^{(29)} f_{0,0}$ denotes the 0 -Fourier coefficient of $f_{0}$, i.e., its $\varphi$-average. 
Now, it is not difficult to check that, choosing $c_{*}$ big enough and letting $\varepsilon$ be small enough, assumptions (151) and (152) are met. In fact, observing that $f_{0}$ in (96)-(97) is such that

$$
\left\|f_{0}\right\|_{r, \rho, s} \leq \text { const } \varepsilon
$$

(152) follows from last equality in (159), by choosing $c_{*}$ large enough. As for (151), observe that for any point in $I_{0} \in U_{r}$ there is a point $I_{0}^{*} \in U$ at distance less than $r$ from it. Hence by the definitions of $\alpha_{0}$ and $r$ and by Cauchy estimates ${ }^{(30)}$, for any $I_{0} \in U_{r}$ and any $0<|k| \leq K$,

$$
\begin{aligned}
\left|h_{0}^{\prime}\left(I_{0}\right) \cdot k\right| & \geq\left|h_{0}^{\prime}\left(I_{0}^{*}\right) \cdot k\right|-\left|h_{0}^{\prime}\left(I_{0}^{*}\right)-h_{0}^{\prime}\left(I_{0}\right)\right||k| \\
& \geq \alpha_{0}-\sup _{\mathcal{I}_{\sigma_{0}}}\left|h_{0}^{\prime \prime}\right| r K \\
& =\alpha_{0} / 2,
\end{aligned}
$$

which proves also (151). Thus, by Proposition 7.1, there exists a real analytic symplectic transformation

$\Psi:\left(I^{\prime}, \varphi^{\prime}, p^{\prime}, q^{\prime}\right) \in \mathcal{D}_{1}:=U_{\frac{r}{2}} \times \mathbb{T}_{\frac{s}{6}}^{2} \times D_{\frac{\rho}{2}}^{4} \rightarrow\left(I_{0}, \varphi_{0}, p_{0}, q_{0}\right) \in U_{r} \times \mathbb{T}_{s_{0}}^{2} \times D_{\rho_{0}}^{4} \subset \mathcal{D}_{0}$,

such that

$$
\begin{aligned}
& \left|I^{\prime}-I_{0}\right| \leq \operatorname{const} \frac{\varepsilon}{\alpha_{0}}=\text { const } \frac{\sqrt{\varepsilon}}{\log \frac{1}{\varepsilon}} \\
& \left|p^{\prime}-p_{0}\right|, \quad\left|q^{\prime}-q_{0}\right| \leq \text { const } \frac{\varepsilon}{\alpha_{0} \rho}=\text { const } \frac{\sqrt{\varepsilon}}{\log \frac{1}{\varepsilon}},
\end{aligned}
$$

and which casts the Hamiltonian $\mathcal{H}_{0}$ into $\mathcal{H}^{\prime}:=\mathcal{H}_{0} \circ \Psi$ with

$$
\mathcal{H}^{\prime}\left(I^{\prime}, \varphi^{\prime}, p^{\prime}, q^{\prime}\right):=h\left(I^{\prime}\right)+g\left(I^{\prime}, p^{\prime}, q^{\prime}\right)+f_{*}\left(I^{\prime}, \varphi^{\prime}, p^{\prime}, q^{\prime}\right),
$$

where (since, by (97), $f_{0,0}$ coincides with $\varepsilon f_{1}(I, p, q)$ )

$$
\begin{aligned}
\sup _{\left(I^{\prime}, p^{\prime}, q^{\prime}\right) \in U_{r / 2} \times V_{\rho / 2}}\left|g_{0}-\varepsilon f_{1}\right| & \leq \text { const } \frac{\varepsilon^{2}}{\alpha_{0} r}=\text { const } \frac{\varepsilon}{\log (1 / \varepsilon)}, \\
\left\|f_{*}\right\|_{r / 2, \rho / 2, s / 6} & \leq \text { const } \varepsilon e^{-K s / 6} \leq \operatorname{const} \varepsilon^{N} .
\end{aligned}
$$

Thus, setting $g_{0}=: \varepsilon \bar{g}, f_{*}=: \varepsilon^{N} \bar{f}$, we see that $\mathcal{H}^{\prime}$ can be rewritten as

$$
\begin{aligned}
\mathcal{H}^{\prime} & :=h\left(I^{\prime}\right)+\varepsilon \bar{g}\left(I^{\prime}, p^{\prime}, q^{\prime}\right)+\varepsilon^{N} \bar{f}\left(I^{\prime}, \varphi^{\prime}, p^{\prime}, q^{\prime}\right), \\
\bar{g} & =f_{1}\left(I^{\prime}, p^{\prime}, q^{\prime}\right)+\frac{1}{\log (1 / \varepsilon)} \bar{f}_{1}\left(I^{\prime}, p^{\prime}, q^{\prime}\right)
\end{aligned}
$$

with $\bar{f}$ and $\bar{f}_{1}$ real-analytic on $\mathcal{D}_{1}$ (compare (158)).

(30) "Cauchy estimates" allow to bound $n$-derivatives of analytic functions on a set $A$ in terms of their sup-norm on larger domains $A^{\prime} \supset A$ divided by dist $\left(\partial A, \partial A^{\prime}\right)^{n}$. 
We now look for elliptic equilibria of the Hamiltonian $\bar{g}$ in (163). Set

$$
G\left(I^{\prime}, p^{\prime}, q^{\prime}\right):=\left(\partial_{p^{\prime}} \bar{g}\left(I^{\prime}, p^{\prime}, q^{\prime}\right), \partial_{q^{\prime}} \bar{g}\left(I^{\prime}, p^{\prime}, q^{\prime}\right)\right) .
$$

Recalling (163) and the definition of $f_{1}$ in (97), we see that, by (99),

$$
\begin{gathered}
\left.G\left(I^{\prime}, 0,0\right)\right|_{\varepsilon=0}=0 \\
\text { and }\left.\operatorname{det} \partial_{\left(p^{\prime}, q^{\prime}\right)} G\left(I^{\prime}, 0,0\right)\right|_{\varepsilon=0}=16\left(\bar{\Omega}_{1} \bar{\Omega}_{2}\right)^{2}>0, \forall I^{\prime} \in U_{r / 2} .
\end{gathered}
$$

Therefore, by the Implicit Function Theorem, we infer that, for any $I^{\prime} \in U_{r / 2}$ and for $\varepsilon$ small enough, there exist real-analytic functions, so that

and

$$
I^{\prime} \in U_{r / 2} \rightarrow\left(p^{\prime}\left(I^{\prime}, \varepsilon\right), q^{\prime}\left(I^{\prime}, \varepsilon\right)\right) \in B_{\text {const } / \log \frac{1}{\varepsilon}} \subset B_{\rho / 2},
$$

$$
\partial_{p^{\prime}} \bar{g}\left(I^{\prime}, p^{\prime}\left(I^{\prime}, \varepsilon\right), q^{\prime}\left(I^{\prime}, \varepsilon\right)\right)=0=\partial_{q^{\prime}} \bar{g}\left(I^{\prime}, p^{\prime}\left(I^{\prime}, \varepsilon\right), q^{\prime}\left(I^{\prime}, \varepsilon\right)\right) .
$$

For $\varepsilon$ small enough, we can consider the following analytic symplectic transformation, which leaves fixed the $I^{\prime}$-variable and is $O\left(\frac{1}{\log \frac{1}{\varepsilon}}\right)$-close to the identity ${ }^{(31)}$,

$\Phi^{\prime}:\left(J^{\prime}, \psi^{\prime}, v^{\prime}, u^{\prime}\right) \in U_{r / 2} \times \mathbb{T}_{s / 7}^{2} \times D_{\rho / 3} \mapsto\left(I^{\prime}, \varphi^{\prime}, p^{\prime}, q^{\prime}\right) \in U_{r / 2} \times \mathbb{T}_{s / 6}^{2} \times D_{\rho / 2}^{4}$, given by

$$
\begin{aligned}
& I^{\prime}=J^{\prime} \\
& \varphi^{\prime}=\psi^{\prime}+p^{\prime}\left(J^{\prime}, \varepsilon\right) \partial_{I^{\prime}} q^{\prime}\left(J^{\prime}, \varepsilon\right)+\partial_{I^{\prime}} q^{\prime}\left(J^{\prime}, \varepsilon\right) v^{\prime}-\partial_{I^{\prime}} p^{\prime}\left(J^{\prime}, \varepsilon\right) u^{\prime}, \\
& p^{\prime}=v^{\prime}+p^{\prime}\left(J^{\prime}, \varepsilon\right) \\
& q^{\prime}=u^{\prime}+q^{\prime}\left(J^{\prime}, \varepsilon\right) .
\end{aligned}
$$

In view of (164), the new Hamiltonian $\hat{\mathcal{H}}:=\mathcal{H}^{\prime} \circ \Phi^{\prime}$ has the form

$$
\hat{\mathcal{H}}\left(J^{\prime}, \psi^{\prime}, v^{\prime}, u^{\prime}\right)=h\left(J^{\prime}\right)+\varepsilon \hat{g}\left(J^{\prime}, v^{\prime}, u^{\prime}\right)+\varepsilon^{N} \hat{f}\left(J^{\prime}, \psi^{\prime}, v^{\prime}, u^{\prime}\right),
$$

with $\hat{f}$ and $\hat{g}$ analytic in $U_{r / 2} \times \mathbb{T}_{s / 7}^{2} \times D_{\rho / 3}$ and

$$
\partial_{v^{\prime}, u^{\prime}} \hat{g}\left(J^{\prime}, 0,0\right)=\partial_{p^{\prime}, q^{\prime}} \bar{g}\left(I^{\prime}, p^{\prime}\left(I^{\prime}, \varepsilon\right), q^{\prime}\left(I^{\prime}, \varepsilon\right)\right)=0, \quad \forall I^{\prime} \in U_{r / 2} .
$$

Also, the eigenvalues of the symplectic quadratic part of $\hat{g}$ are given by ${ }^{(31)} \Phi^{\prime}$ has generating function $J^{\prime} \cdot \varphi^{\prime}+\left(v^{\prime}+p^{\prime}\left(J^{\prime}, \varepsilon\right)\right) \cdot\left(q^{\prime}-q^{\prime}\left(J^{\prime}, \varepsilon\right)\right)$. 
$\pm \mathrm{i} \hat{\Omega}_{j}\left(J^{\prime}\right)$, for $j=1,2$, where

$$
\hat{\Omega}_{j} \in \mathbb{R} \quad \text { and } \quad\left|\hat{\Omega}_{j}-\bar{\Omega}_{j}\right| \leq \mathrm{const} \frac{1}{\log \frac{1}{\varepsilon}} .
$$

Thus, by a well known result by Weierstrass on the symplectic diagonalization of quadratic Hamiltonians, we can find an analytic transformation $O\left(\frac{1}{\log \frac{1}{\varepsilon}}\right)-$ close to the identity

$$
\check{\Phi}:(J, \psi, v, u) \in U_{r / 2} \times \mathbb{T}_{s / 8}^{2} \times D_{\rho / 4}^{4} \mapsto\left(J^{\prime}, \psi^{\prime}, v^{\prime}, u^{\prime}\right) \in U_{r / 2} \times \mathbb{T}_{s / 7}^{2} \times D_{\rho / 3}^{4},
$$

so that $J^{\prime}=J$ and the quadratic part of $\hat{g}$ becomes, simply, $\sum_{j=1}^{2} \hat{\Omega}_{j}(J)\left(u_{j}^{2}+\right.$ $v_{j}^{2}$ ). Whence, the Hamiltonian $\hat{\mathcal{H}}$ takes the form $\check{\mathcal{H}}:=\hat{\mathcal{H}} \circ \check{\Phi}$, with

(166) $\check{\mathcal{H}}(J, \psi, v, u)=h_{\varepsilon}(J)+\varepsilon \sum_{j=1}^{2} \hat{\Omega}_{j}(J)\left(u_{i}^{2}+v_{i}^{2}\right)+\varepsilon \widetilde{g}(J, v, u)+\varepsilon^{N} \widetilde{f}(J, \psi, v, u)$, where

$$
h_{\varepsilon}(J):=h_{0}(J)+\varepsilon \hat{g}(J, 0,0),
$$

$\widetilde{g}, \widetilde{f}, \hat{\Omega}_{j}$ are real-analytic for $(J, \psi, v, u)$ in

$$
\mathcal{D}_{2}:=U_{r / 2} \times \mathbb{T}_{s / 8}^{2} \times D_{\rho / 4}^{4}
$$

and

$$
\sup _{J \in U_{r / 2}}|\widetilde{g}(J, v, u)| \leq \text { const }|(v, u)|^{3} .
$$

Because of (165), the non-degeneracy condition (99) implies (for $\varepsilon$ small enough)

$$
\begin{aligned}
& \inf _{J \in U} \hat{\Omega}_{i} \geq \inf _{J \in U_{r / 2}}\left|\hat{\Omega}_{i}\right| \geq \text { const }>0, \\
& \inf _{J \in U}\left(\hat{\Omega}_{2}-\hat{\Omega}_{1}\right) \geq \inf _{J \in U_{r / 2}}\left|\hat{\Omega}_{2}-\hat{\Omega}_{1}\right| \geq \text { const }>0 .
\end{aligned}
$$

Setting $\widetilde{\Omega}_{j}:=2 \hat{\Omega}_{j}$ for $j=1,2$, introducing complex coordinates

$$
z=\frac{v+\mathrm{i} u}{\sqrt{2}}, \quad \bar{z}=\frac{v-\mathrm{i} u}{\sqrt{2}}
$$

and defining

$$
\begin{aligned}
g(J, z, \bar{z}) & :=\widetilde{g}\left(J, \frac{z+\bar{z}}{\sqrt{2}}, \frac{z-\bar{z}}{\mathrm{i} \sqrt{2}}\right) \\
\text { and } \quad f(J, \psi, z, \bar{z}) & :=\widetilde{f}\left(J, \psi, \frac{z+\bar{z}}{\sqrt{2}}, \frac{z-\bar{z}}{\mathrm{i} \sqrt{2}}\right),
\end{aligned}
$$

we obtain (101). Finally (102), (106), (107), follows from (158), (169), (170), respectively. 


\section{REFERENCES}

[Am] A. Ambrosetti, Critical points and nonlinear variational problems, Mm. Soc. Math. Fr., Nouv. Sr. 49, 1992.

[ACE] A. Ambrosetti - V. Coti-Zelati - I. Ekeland, Symmetry breaking in Hamiltonian systems, J. Differential Equation 67 (1987), 165-184.

[AB] A. Ambrosetti - M. Badiale, Homoclinics: Poincaré-Melnikov type results via a variational approac, Ann. Inst. H. Poincaré - Analyse Non Linéaire 15, n.2 (1998), 233-252.

[A] V. I. ARNOLD, Small denominators and problems of stability of motion in classical and celestial mechanics, Uspekhi Mat. Nauk 18 (1963), 91-192.

[BK] D. BERNSTEIN - A. KATOK, Birkhoffperiodic orbits for small perturbations of completely integrable Hamiltonian systems with convex Hamiltonians, Invent. Math. 88 (1987), 225241.

[BBB] M. BERTI - L. BIASCO - P. Bolle, Drift in phase space, a new variational mechanism with optimal diffusion time, J. Math. Pures Appl. 82 (2003), 613-664.

[BB] M. BERTI - P. Bolle,, A functional analysis approach to Arnold Diffusion, Ann. Inst. H. Poincaré Anal. Non Linéaire 19 (2002), 395-450.

[BCV] L. Biasco - L. Chierchia - E. Valdinoci, Elliptic two-dimensional invariant tori for the planetary three-body problem, Arch. Ration. Mech. Anal. 170 (2003), 91-135.

[B] G. D. BIRKHOFF, Une generalization á n-dimensions du dernier théorème de géometrié de Poincaré, C. R. Acad. Sci. Paris Sér. I Math. 192 (1931), 196-198.

[BL] G. D. BirkhofF - D. C. LewIS, On the periodic motions near a given periodic motion of a dynamical system, Ann. Mat. Pura Appl., IV. Ser. 12 (1933), 117-133.

[Bo] J. Bourgain, On Melnikov's persistency problem, Math. Res. Lett. 4 (1997), 445-458.

[BHS] H. W. Broer - G. B. Huitema - M. B. Sevriuk, "Quasi periodic motions in families of dynamical systems", Lecture Notes in Math. 1645, Springer, 1996.

[CZ] C. Conley - E. Zehnder, "An index theory for periodic solutions of a Hamiltonian system", Lecture Notes in Mathematics 1007, Springer, 1983, 132-145.

[CZ1] C. CONLEy - E. ZeHnder, The Birkhoff-Lewis fixed point theorem and a conjecture of V. I. Arnold, Invent. Math. 73 (1983), 33-49.

[E] L. Eliasson, Perturbations of stable invariant tori for Hamiltonian systems, Ann. Scuola Norm. Sup. Pisa Cl. Sci. (4), 15 (1988), 115-147.

[J] W. H. JEFFERYs, Periodic orbits in the three-dimensional three-body problem, Astronom. J. 71 (1966), 566-567.

[JM] W. H. JEFFERYs - J. Moser, Quasi-periodic solutions for the three-body problem, Astronom. J. 71 (1966), 568-578.

[JV] Á. Jorba - J. Villanueva, On the Normal Behaviour of Partially Elliptic Lower Dimensional Tori of Hamiltonian Systems, Nonlinearity 10 (1997), 783-822.

[K] S. B. KuKsin, Perturbation theory of conditionally periodic solutions of infinite-dimensional Hamiltonian systems and its applications to the Korteweg-de Vries equation, Mat. Sb. (N.S.) 136 (178) (1988), 396-412, 431; translation in Math. USSR-Sb. 64 (1989), 397-413.

[LR] J. LASKAR - P. RoBUtel, Stability of the planetary three-body problem. I. Expansion of the planetary Hamiltonian, Celestial Mech. Dynam. Astronom. 62 (1995), 193-217. 
[L] D. C. Lewis, Sulle oscillazioni periodiche di un sistema dinamico, Atti Accad. Naz. Rend. Cl. Sci. Fis. Mat. Natur. Rend. Lincei (9) Mat. Appl. 19 (1934), 234-237.

[M] V. K. Melnikov, On certain cases of conservation of almost periodic motions with a small change of the Hamiltonian function, Dokl. Akad. Nauk SSSR 165 (1965), 12451248.

[Mo] J. Moser, "Proof of a generalized form of a fixed point Theorem due to G. D. Birkhoff", In: Geometry and topology (Proc. III Latin Amer. School of Math., Inst. Mat. Pura Aplicada CNP, Rio de Janeiro, 1976), 464-494. Lecture Notes in Math., Vol. 597, Springer, Berlin, 1977.

[P] H. Poincaré, “Les Méthodes nouvelles de la Mécanique Céleste”, Gauthier Villars, Paris, 1892.

[Pö] J. Pöschel, Integrability of Hamiltonian systems on Cantor sets, Comm. Pure Appl. Math. 35 (1982), 653-696.

[Pö1] J. Pöschel, On elliptic lower dimensional tori in Hamiltonian system, Math. Z. 202 (1989), 559-608.

[Pö2] J. Pöschel, A KAM-Theorem for some nonlinear PDEs, Ann. Scuola Norm. Sup. Pisa Cl. Sci. (4) 23 (1996), 119-148.

[R] P. RoBUtEL, Stability of the planetary three-body problem. II. KAM theory and existence of quasiperiodic motions, Celestial Mech. Dynam. Astronom. 62 (1995), 219-261.

[W] C. E. WAYNE, Periodic and quasi-periodic solutions of nonlinear wave equations via KAM theory, Comm. Math. Phys. 127 (1990), 479-528.

[XY] J. XU - J. You, Persistence of lower-dimensional tori under the first Melnikov's nonresonance condition, J. Math. Pures Appl. 80 (2001), 1045-1067.

Settore di Analisi Funzionale e Applicazioni Scuola Internazionale Superiore di Studi Avanzati (SISSA)

Via Beirut 2-4

34014 Trieste (Italy)

berti@sissa.it

Dipartimento di Matematica

Università "Roma Tre"

Largo S. L. Murialdo 1

00146 Roma (Italy)

biasco@mat.uniroma3.it

Dipartimento di Matematica

Università di Roma "Tor Vergata"

Via della Ricerca Scientifica 1

00133 Roma (Italy)

valdinoci@mat.uniroma2.it 